\title{
Functoriality of Khovanov homology
}

Pierre Voge 1

\begin{abstract}
In this paper we prove that every Khovanov homology associated to a Frobenius algebra of rank 2 can be modified in such a way as to produce a TQFT on oriented links, that is a monoidal functor from the category of cobordisms of oriented links to the homotopy category of complexes.
\end{abstract}

Keywords: Frobenius algebra, Khovanov homology, cobordism of oriented links, monoidal functor.

Mathematics Subject Classification (2010): 13Axx, 13Dxx, 57M25

\section{Introduction.}

The Khovanov homology was introduced by Khovanov [Kh1] as a categorification of the Jones polynomial. The main ingredient of this homology is a monoidal functor from the category of cobordisms of oriented 1-manifolds to a category of modules. But such monoidal functors are characterized by commutative Frobenius algebras [Ko]. Therefore the Khovanov homology can be defined for every commutative Frobenius algebra $R$. The classical Khovanov homology corresponds to the case: $R=\mathbf{Z}[\alpha] /\left(\alpha^{2}\right)$ and the Lee-version of this homology corresponds to the case: $R=\mathbf{Z}[\alpha] /\left(\alpha^{2}-1\right)$.

An important problem in this theory is to extend the Khovanov homology to a monoidal functor from the category of cobordisms of oriented links. The first attempt by Khovanov gave a negative answer because of many problems of signs. Functoriality up to sign was conjectured by Khovanov and proved later by Jacobson [Ja], Bar Natan [BN2] and Khovanov [Kh3]. This functoriality up to sign was used by Rasmussen [Ra1] to prove a conjecture of Milnor about the slice genus. Strict functoriality for alternative versions of Khovanov homology were proven by Blanchet [Bl] and Clark, Morrison, Walker [CMW]. In these versions of Khovanov homology, the Kauffman bracket is replaced with the $s l_{2}$-polynomial in [Bl] and the $s u_{2}$-polynomial in [CMW].

In this paper we will prove functoriality for the Khovanov homology associated to any Frobenius algebra of rank 2 (and the classical Kauffman bracket).

Suppose $K$ is a commutative ring and $R$ is a Frobenius $K$-algebra of rank 2 . Denote by $u \mapsto \bar{u}$ the involution of the extension $K \subset R$ and by $\delta$ the image of 1

\footnotetext{
${ }^{1}$ Université Paris Diderot, Institut de Mathématiques de Jussieu-Paris Rive Gauche (UMR 7586), Bâtiment Sophie Germain, Case 7012, 75205-Paris Cedex 13 - Email: pierre.vogel@imj-prg.fr
} 
under the composite map:

$$
R \stackrel{\text { coproduct }}{\longrightarrow} R \otimes R \stackrel{\text { product }}{\longrightarrow} R
$$

Denote by $\mathscr{L}$ the category of cobordisms of oriented links in $\mathbf{R}^{3}$ and by $\mathscr{C}_{K}$ the homotopy category of $K$-complexes. These categories are both monoidal. The main result of this paper is the following:

Theorem A: There exists a monoidal functor $\Psi$ from $\mathscr{L}$ to $\mathscr{C}_{K}$ satisfying the following property:

for any diagram $D$ of an oriented link $L, \Psi(L)$ is isomorphic to the classical Khovanov complex of $D$.

Such functors are not unique. The construction produces a lot of functors (called Khovanov functors) satisfying this property. There is a well defined invertible element in $R$ associated to each Khovanov functor: its weight. Actually if $C$ is an unknotted cobordism of genus 1 from the unknot diagram (without any crossing) to itself, the image of this cobordism under a Khovanov functor of weight $\pi$ is the multiplication by $\delta / \pi$ (resp. $\bar{\delta} / \bar{\pi}$ ) from $R$ to $R$ if the unknot is oriented clockwise (resp. counterclockwise).

Theorem B: For every invertible element $\pi \in R$ there is a Khovanov functor of weight $\pi$. Moreover two Khovanov functors with the same weight are isomorphic.

Consider a closed oriented surface $S$ in $\mathbf{R}^{4}$. This surface may be consider as a cobordism from the empty link to itself. Therefore every Khovanov functor $\Psi$ induces an invariant $\Psi(S) \in K$. In the classical case the functor $\Psi$ was well defined up to sign and $\Psi(S)$ was determined by Tanaka [Ta] and Rasmussen [Ra2], at least for connected surfaces. They prove that $\Psi(S)$ doesn't depend on the embedding $S \subset \mathbf{R}^{4}$. This result is still true in the general case and $\Psi(S)$ depends only on $S$ and the weight of $\Psi$ and not on the embedding. More precisely we have the following result:

Theorem C: Let $\Psi$ be a Khovanov functor of weight $\pi \in R^{*}$ and $S$ be a closed oriented surface in $\mathbf{R}^{4}$. Then we have:

$$
\Psi(S)=\prod_{i} \varepsilon\left(\delta^{p_{i}} \pi^{1-p_{i}}\right)
$$

where the $p_{i}$ 's are the genus of the components of $S$.

\section{Frobenius algebras of rank 2 .}

1.1 Definition: Let $K$ be a commutative ring. A $K$-algebra of rank 2 is a $K$-algebra isomorphic to $K[\alpha] /(P)$, where $P \in K[\alpha]$ is a monic polynomial of degree 2 .

The element $\alpha$ is called a $K$-generator of $R$ and the involution of the extension $K \subset R$ is called the involution of $R$. 
Let $R$ be a $K$-algebra of rank 2 and $\alpha$ be a $K$-generator of $R$. The polynomial $P$ is given by:

$$
P(\alpha)=\alpha^{2}-s \alpha+p
$$

with $s$ and $p$ in $K$ and the involution of $R$, denoted by $u \mapsto \bar{u}$, is the identity on $K$ and sends $\alpha$ to $\bar{\alpha}=s-\alpha$. So we have:

$$
s=\alpha+\bar{\alpha} \quad p=\alpha \bar{\alpha}
$$

and, for any $u \in R, u+\bar{u}$ and $u \bar{u}$ are in $K$.

1.2 Proposition: Let $K$ be a commutative ring and $R$ be a Frobenius $K$-algebra. Suppose $R$ is a $K$-algebra of rank 2 and $\alpha$ is a $K$-generator of $R$. Then there is a unique invertible element $\omega$ in $R$ such that the coproduct and the counit are defined by:

$$
\begin{gathered}
\forall u \in R, \quad \Delta(u)=u \otimes \omega \alpha-u \bar{\alpha} \otimes \omega \\
\varepsilon(\omega)=0 \quad \varepsilon(\omega \alpha)=1
\end{gathered}
$$

$\omega$ is called the twisting element of $R$.

Moreover, if $\omega$ is any invertible element in $R$, the coproduct and the counit defined by the formulae above induce a structure of Frobenius $K$-algebra on $R$.

Proof: Since $\Delta$ is a $K$-linear map from $R$ to $R \otimes R$, there exist two $K$-linear map $f$ and $g$ from $R$ to $R$ such that:

$$
\forall u \in R, \quad \Delta(u)=f(u) \otimes 1+g(u) \otimes \alpha
$$

Since $R$ is a Frobenius algebra we have the relation: $\Delta(u)=(u \otimes 1) \Delta(1)$ and then:

$$
\forall u \in R, \quad f(u)=u f(1) \quad g(u)=u g(1)
$$

We have also the relation: $(\alpha \otimes 1-1 \otimes \alpha) \Delta(1)=0$ which implies: $f(1)+\bar{\alpha} g(1)=0$. By setting: $\omega=g(1)$ we get:

$$
\Delta(u)=u \omega \otimes \alpha-u \omega \bar{\alpha} \otimes 1=u \otimes \omega \alpha-u \bar{\alpha} \otimes \omega
$$

On the other hand the counit satisfies the relation: $(1 \otimes \varepsilon) \Delta(u)=u$ which is equivalent to:

$$
\varepsilon(\omega)=0 \quad \varepsilon(\omega \alpha)=1
$$

The last thing to do is to prove that $\omega$ is invertible in $R$. Set: $u=\varepsilon(\alpha)-\bar{\alpha} \varepsilon(1)$. It is easy to see the following:

$$
\varepsilon(\omega u)=\varepsilon(1) \quad \varepsilon(\omega u \alpha)=\varepsilon(\alpha)
$$

So for any $v \in R$, we have: $\varepsilon((\omega u-1) v)=0$. Set: $\omega u-1=a+b \alpha$ with $a$ and $b$ in $K$. Testing this formula with $v=\omega$ and $v=\omega \alpha$ implies: $b=a=0$. Therefore $\omega$ is invertible with inverse $u$.

The last part of the proposition is easy to check. 
1.3 Remarks: Let $R_{0}$ be the ring $\mathbf{Z}\left[\alpha, \bar{\alpha}, a, b,(a+b \alpha)^{-1},(a+b \bar{\alpha})^{-1}\right]$. This ring is equipped with an involution keeping $a$ and $b$ fixed and exchanging $\alpha$ and $\bar{\alpha}$. The ring of invariant elements under this involution is:

$$
K_{0}=\mathbf{Z}\left[\alpha+\bar{\alpha}, \alpha \bar{\alpha}, a, b,((a+b \alpha)(a+b \bar{\alpha}))^{-1}\right]
$$

and $R_{0}$ is a $K_{0}$-algebra of rank 2 with $K_{0}$-generator $\alpha$. By setting: $\omega=a+b \alpha$, we see that $R_{0}$ is a Frobenius algebra with twisting element $\omega$. Moreover $R_{0}$ is universal in the following sense:

Let $R$ be a Frobenius $K$-algebra of rank 2 and $\beta$ be a $K$-generator of $R$. Then there exists a unique Frobenius algebra homomorphism from $R_{0}$ to $R$ sending $\alpha$ to $\beta$.

In particular the endomorphisms of the Frobenius algebra $R_{0}$ are ring homomorphisms characterized by:

$$
\begin{array}{rlrl}
\alpha \mapsto \lambda \alpha+\mu & \bar{\alpha} \mapsto \lambda \bar{\alpha}+\mu \\
a \mapsto \lambda^{-1} a-\lambda^{-2} \mu b & b \mapsto \lambda^{-2} b
\end{array}
$$

where $(\lambda, \mu)$ is any element of $K_{0}^{*} \times K_{0}$.

Another description of the universal algebra $R_{0}$ was founded by Khovanov in $[\mathrm{Kh} 4]$.

1.4 From now on $K$ will be a commutative ring, $R$ a Frobenius $K$-algebra of rank 2 and $\alpha$ a $K$-generator of $R$. The twisting element in $R$ will be denoted by $\omega$. Set:

$$
t=\varepsilon(1) \quad \delta=\omega(\alpha-\bar{\alpha}) \quad \theta=\frac{\omega}{\bar{\omega}}
$$

It is easy to see that these elements do not depend on the choice of $\alpha$. Moreover we have the following relations:

$$
t \delta=1-\theta \quad \bar{t}=t \quad \bar{\delta}=-\theta^{-1} \delta \quad \bar{\theta}=\theta^{-1}
$$

Set: $A=\mathbf{Z}\left[t, \delta,(1-t \delta)^{-1}\right]$. This ring is a ring with involution and it is contained in the algebra $R_{0}$. Therefore every Frobenius algebra of rank 2 is an $A$-algebra.

By setting: $s=\delta \bar{\delta}=-\theta^{-1} \delta^{2}=-(1-t \delta)^{-1} \delta^{2}$, it is not difficult to see that the ring of elements in $A$ that are fixed by the involution is $\mathbf{Z}[s, t]$ and $A$ is a $\mathbf{Z}[s, t]$-algebra of rank 2 with $\mathbf{Z}[s, t]$-generator $\delta$.

1.5 Lemma: The ring of elements in $R_{0}$ which are invariant under every endomorphism of $R_{0}$ is the ring $A$.

Proof: Suppose that $a_{1}, \ldots, a_{p}$ are elements in a commutative ring $\Lambda$. Then denote by $\Lambda<a_{1}, \ldots, a_{p}>$ the ring obtained by inverting $a_{1}, \ldots, a_{p}$ in $\Lambda$. So we have:

$$
R_{0}=\mathbf{Z}[\alpha, \bar{\alpha}, a, b]<a+b \alpha, a+b \bar{\alpha}>
$$


We have the following:

$$
\omega=a+b \alpha \quad t=\varepsilon(1)=\frac{-b}{\omega \bar{\omega}} \quad \bar{\omega}(1-t \delta)=\omega
$$

and then

$$
\begin{aligned}
& \left.R_{0}=\mathbf{Z}[\alpha, \alpha-\bar{\alpha}, b, \omega]<\omega, \bar{\omega}\right)>=\mathbf{Z}[\alpha, \delta, b, \omega]<\omega, \bar{\omega}> \\
& =\mathbf{Z}[\alpha, t, \delta, \omega]<\omega, 1-t \delta>=(\mathbf{Z}[\alpha, t, \delta]<1-t \delta>)\left[\omega^{ \pm}\right]
\end{aligned}
$$

Consider the endomorphisms of $R_{0}$ sending $\alpha$ to $\alpha+\mu$, for some $\mu \in K_{0}$. These endomorphisms keep $t, \delta$ and $\omega$ fixed. Then the ring $R_{1}$ of elements in $R_{0}$ which are invariant under these endomorphisms is:

$$
R_{1}=\mathbf{Z}[t, \delta, \omega]<\omega, 1-t \delta>=(\mathbf{Z}[t, \delta]<1-t \delta>)\left[\omega^{ \pm}\right]
$$

But every endomorphism of $R_{0}$ keeps $t$ and $\delta$ fixed, and multiplies $\omega$ by any element $\lambda \in K_{0}^{*}$. Since $K_{0}^{*}$ contains any power of $\omega \bar{\omega}$, the ring $R_{2}$ of elements in $R_{0}$ which are invariant under every endomorphism of $R_{0}$ is:

$$
R_{2}=\mathbf{Z}[t, \delta]<1-t \delta>=A
$$

Denote by $\mathscr{C}$ the category of cobordisms of oriented curves (i.e. closed oriented 1-dimensional manifolds). The disjoint union induces on $\mathscr{C}$ a monoidal structure. TQFT's for oriented surfaces are in one to one correspondance with commutative Frobenius algebras $[\mathrm{Ko}]$. In particular the Frobenius algebra $R$ induces a monoidal functor $\Phi$ from $\mathscr{C}$ to the category of $K$-modules. This functor has the following properties: it sends $\emptyset$ to $K$ and $S^{1}$ to $R$, unit, counit, product and coproduct of $R$ are the image under $\Phi$ of suitable cobordisms and, for every oriented closed surface $\Sigma, \Phi(\Sigma)$ is an element of $K$. An easy computation gives the following:

1.6 Lemma: For every $p \geq 0$ denote by $\Sigma_{p}$ an oriented surface of genus $p$. Then we have the following:

$$
\begin{aligned}
\Phi\left(\Sigma_{p}\right) & =\varepsilon\left(\delta^{p}\right) \\
\sum_{p \geq 0} x^{p} \Phi\left(\Sigma_{p}\right)=\varepsilon\left(\frac{1}{1-x \delta}\right) & =\frac{t+x\left(2-t^{2} s\right)}{1-x t s+x^{2} s} \in K[[x]]
\end{aligned}
$$

Remark: If $R$ is the ring $R_{0}$ and $S$ is a closed oriented surface, $\Phi(S)$ is an element of $\mathbf{Z}[t, s]$ of degree $\chi(S)$ where the degree in $\mathbf{Z}[t, s]$ is defined by: $\partial^{\circ} t=2, \partial^{\circ} s=-4$.

Remark: The Khovanov homology is defined by using a Frobenius algebra $R$. The classical Khovanov homology corresponds to the case: $R=\mathbf{Z}[\alpha] /\left(\alpha^{2}\right)$ and the Lee version of the Khovanov homology corresponds to: $R=\mathbf{Z}[\alpha] /\left(\alpha^{2}-1\right)$. In both cases, we have:

$$
\omega=1 \quad t=0 \quad \theta=1 \quad \delta=2 \alpha \quad s=-4 \alpha^{2}
$$


1.7 The category of mixed cobordisms $\mathscr{C}^{\prime}$. The functor $\Phi$ is defined on the category $\mathscr{C}$, but it is possible to extend it to a bigger category $\mathscr{C}^{\prime}$.

First of all, if $S$ is a surface, consider the commutative monoid with unit defined by generators and relations. Generators are pairs $(x, a) \in S \times R$ and the relations are the following:

$$
\begin{aligned}
(x, a)(x, b) & \equiv(x, a b) \\
(x, 1) & \equiv 1
\end{aligned}
$$

An element of this monoid will be called a $R$-marking of $S$.

If $C$ and $C^{\prime}$ are two closed oriented curves, define a mixed cobordism from $C$ to $C^{\prime}$ as a triple $(S, \Gamma, u)$ where:

- $S$ is a compact surface containing a closed curve $\Gamma$ in its interior

$-S \backslash \Gamma$ is oriented with boundary: $\partial(S \backslash \Gamma)=C^{\prime}-C$

— when crossing $\Gamma$ the orientation of $S \backslash \Gamma$ is changed

$-u$ is a $R$-marking of $S \backslash \Gamma$.

The category $\mathscr{C}^{\prime}$ is defined as follows: the objects of $\mathscr{C}^{\prime}$ are the objects of $\mathscr{C}$, that is the oriented curves. A morphism in $\mathscr{C}^{\prime}$ from an oriented curve $C$ to an oriented curve $C^{\prime}$ is the isomorphism class of a mixed cobordism $(S, \Gamma, u)$ from $C$ to $C^{\prime}$. So we get a monoidal category $\mathscr{C}^{\prime}$ containing $\mathscr{C}$.

1.8 Proposition: There is a unique monoidal functor $\Phi^{\prime}$ from $\mathscr{C}^{\prime}$ to the category of $K$-modules satisfying the following:

1) $\Phi^{\prime}$ is an extension of the functor $\Phi$

2) $\Phi^{\prime}\left(S, \Gamma,\left(x_{1}, a_{1}\right)\left(x_{2}, a_{2}\right) \ldots\left(x_{p}, a_{p}\right)\right)$ depends only on the isotopy classes of the $x_{i}$ 's in $S \backslash \Gamma$

3) $\Phi^{\prime}(S, \Gamma,(x, a) u)$ is $K$-linear with respect to $a$.

4) if $(S, \Gamma,(x, a) u)$ is a mixed cobordism and $x^{\prime}$ is obtained by making $x$ go through $\Gamma$, we have: $\Phi^{\prime}(S, \Gamma,(x, a) u)=\Phi^{\prime}\left(S, \Gamma,\left(x^{\prime}, \bar{a}\right) u\right)$

5) $\Phi^{\prime}\left(S^{1} \times[0,1], \emptyset,(x, a)\right)$ is the multiplication by a, from $R=\Phi^{\prime}\left(S^{1}\right)$ to $R$

6) $\Phi^{\prime}\left(S^{1} \times[0,1], S^{1} \times\{1 / 2\}, 1\right)$ is the map $a \mapsto \delta \bar{a}$

7) $\Phi^{\prime}(S, \Gamma, u)$ vanishes if $S$ is not orientable.

Proof: Let $\Phi^{\prime}$ and $\Phi^{\prime \prime}$ be two functors satisfying all these conditions. Because of conditions 1 ) and 5$), \Phi^{\prime}$ and $\Phi^{\prime \prime}$ are the same on mixed cobordisms $(S, \emptyset, u)$. Consider now a mixed cobordism $(S, \Gamma, u)$ from $C$ et $C^{\prime}$. Let $N$ be a small regular neighborough of $\Gamma$ and $S^{\prime}$ be the closure of $S \backslash N$. Then the morphism $(S, \Gamma, u)$ is the composite of $\left(S^{\prime}, \emptyset, u\right)$ from $C$ to $C^{\prime} \coprod \partial N$ and $1 \times(N, \Gamma, 1)$ from $C^{\prime} \coprod \partial N$ to $C^{\prime}$. Therefore, in order to prove that $\Phi^{\prime}$ and $\Phi^{\prime \prime}$ are the same on $(S, \Gamma, u)$ it is enough to prove that $\Phi^{\prime}$ and $\Phi^{\prime \prime}$ are the same on $(N, \Gamma, 1)$. But that's a consequence of conditions 6$)$ and 7$)$. So, if the functor $\Phi^{\prime}$ exists, it is unique.

For the construction of $\Phi^{\prime}$, it is enough to consider the universal case: $R=R_{0}$. Consider a mixed cobordism $(S, \emptyset, u)$ from $C_{0}$ to $C_{1}$. This cobordism is a composite of cobordisms on the form $\left(S^{\prime}, \emptyset, 1\right)$ or $(C \times[0,1], \emptyset, u)$. Because of conditions 1$)$ and $5)$, there is a unique choice for the morphism $\Phi^{\prime}(S, \emptyset, u)$. Moreover properties of $R$ and $\Phi$ imply that this morphism depends only on the cobordism $(S, \emptyset, u)$. 
Consider now any mixed cobordism $(S, \Gamma, u)$ from $C$ et $C^{\prime}$. As before denote by $N$ a small regular neighborough of $\Gamma$ and $S^{\prime}$ be the closure of $S \backslash N$. Then the morphism $(S, \Gamma, u)$ is the composite of $\left(S^{\prime}, \emptyset, u\right)$ from $C$ to $C^{\prime} \coprod \partial N$ and $1 \times(N, \Gamma, 1)$ from $C^{\prime} \coprod \partial N$ to $C^{\prime}$. So to define $\Phi^{\prime}(S, \Gamma, u)$, it's enough to define $\Phi^{\prime}(N, \Gamma, 1): \Phi^{\prime}(\partial N) \rightarrow$ $K$. Let $\Gamma_{i}$ be the component of $\Gamma$ and $N_{i}$ be the component of $N$ containing $\Gamma_{i}$. We must set:

$$
\Phi^{\prime}(N, \Gamma, 1)=\otimes_{i} \Phi^{\prime}\left(N_{i}, \Gamma_{i}, 1\right)
$$

If $N_{i}$ is a Möbius band, we have to set: $\Phi^{\prime}\left(N_{i}, \Gamma_{i}, 1\right)=0$. If not, $N_{i}$ is a band $\Gamma_{i} \times[-1,1]$ and we define the map $\Phi^{\prime}\left(N_{i}, \Gamma_{i}, 1\right)$ from $R \otimes R$ to $K$ by: $a \otimes b \mapsto a \bar{b}+b \bar{a}$.

So we get a monoidal functor from $\mathscr{C}^{\prime}$ to the category of $K$-modules. It is not difficult to check all the conditions except the last one.

Suppose that $(S, \Gamma, u)$ is a mixed cobordism from $C$ to $C^{\prime}$, where $S$ is nonorientable. Since $S$ isn't orientable, there exists a loop $\gamma$ starting at some point $x \in S \backslash \Gamma$ and intersecting transversally $\Gamma$ an odd number of times. If $u$ is a product of $\left(x_{i}, a_{i}\right)$, we may suppose that $\gamma$ doesn't meet any of the $x_{i}$ 's. Let $D$ be a small disk in $S \backslash(\Gamma \cup \gamma)$ near $x$ and $S^{\prime}$ be the closure of $S \backslash D$. Then $(S, \Gamma, u)$ is the composite of $\left(S^{\prime}, \Gamma, u\right)$ from $C$ to $C^{\prime} \coprod S^{1}$ and $1 \times(D, \emptyset, 1)$ from $C^{\prime} \coprod S^{1}$ to $C^{\prime}$ and it is enough to prove that $\Phi^{\prime}\left(S^{\prime}, \Gamma, u\right)$ vanishes.

Because of conditions 3) and 4) we have:

$$
\Phi^{\prime}\left(S^{\prime}, \Gamma,(x, \alpha) u\right)=\Phi^{\prime}\left(S^{\prime}, \Gamma,(x, \bar{\alpha}) u\right) \quad \Longrightarrow \quad \Phi^{\prime}\left(S^{\prime}, \Gamma,(x, \alpha-\bar{\alpha}) u\right)=0
$$

But $\Phi^{\prime}\left(S^{\prime}, \Gamma, u\right)$ is a morphism from $\Phi^{\prime}(C)$ to $\Phi^{\prime}\left(C^{\prime} \coprod S^{1}\right)=\Phi^{\prime}\left(C^{\prime}\right) \otimes R$. So we have:

$$
(1 \otimes(\alpha-\bar{\alpha})) \Phi^{\prime}\left(S^{\prime}, \Gamma, u\right)(v)=0
$$

for any $v \in \Phi^{\prime}(C)$. Since $\alpha-\bar{\alpha}$ is not a zero divisor in $R=R_{0}, \Phi^{\prime}\left(S^{\prime}, \Gamma, u\right)(v)$ vanishes for every $v \in \Phi^{\prime}(C)$ and $\Phi^{\prime}\left(S^{\prime}, \Gamma, u\right)$ is the zero map.

\section{Khovanov complexes of diagrams.}

In this section we'll construct many Khovanov complexes associated to link diagrams (oriented or not). All these complexes are graded (or bigraded) differential $K$-modules (or $R$-modules).

2.1 Notations: Let $X$ be a finite set. Denote by $\Lambda(X)$ the maximal exterior power of the $\mathbf{Z}$-module freely generated by $X$. This module is a free $\mathbf{Z}$-module of rank 1 .

Suppose $X$ is a graded set. The grading if defined by a map $e$ from $X$ to $\mathbf{Z}$ and we get a $\mathbf{Z}$-grading on $\Lambda(X)$ by the rule:

$$
\partial^{\circ}\left(x_{1} \wedge x_{2} \wedge \ldots \wedge x_{p}\right)=\sum_{i} e\left(x_{i}\right)
$$

This graded module will be denoted by $\Lambda^{e}(X)$. If $e$ is the map $x \mapsto 1$ (resp. $x \mapsto-1$ ), $\Lambda^{e}(X)$ will be denoted by $\Lambda^{+}(X)\left(\operatorname{resp} . \Lambda^{-}(X)\right)$. 


\subsection{The Khovanov complex $k h(D)$.}

Let $D$ be a link diagram and $X$ be the set of crossings of $D$. Denote by $\widehat{X}$ the set of maps $s: X \rightarrow\{ \pm 1\}$. Such a map is called a state on $D$.

If $s \in \widehat{X}$ is a state, we can modify $D$ near each crossing $x$ by the rule:

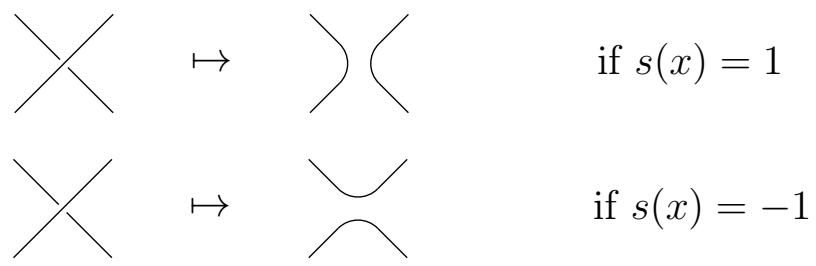

So we get a new diagram $D_{s}$ called the $s$-resolution of $D$. This diagram is a curve embedded in the plane and there is a unique compact $K \subset \mathbf{R}^{2}$ such that $D_{s}$ is the boundary of $K$. Since $K$ is oriented by the plane, $D_{s}$ is an oriented curve. Denote by $X_{s}$ the set $s^{-1}(-1)$. So for every state $s$ we have an oriented curve $D_{s}$ and a set $X_{s}$.

Consider the following graded $K$-module:

$$
E=\oplus_{s} \Lambda^{-}\left(X_{s}\right) \otimes \Phi\left(D_{s}\right)
$$

Let $x$ be a crossing of $D$. By sending $x$ to -1 and all the other crossings to 1 we get a state $e_{x}$. If $s$ is a state denote by $s^{\prime}$ the state $e_{x} s$. The manifold $D_{s^{\prime}}$ is obtained from $D_{s}$ by a surgery along a curve $\gamma_{x}$ near $x$. So we get an oriented cobordism from $D_{s}$ to $D_{s^{\prime}}$ and, via the functor $\Phi$, a map from $\Phi\left(D_{s}\right)$ to $\Phi\left(D_{s^{\prime}}\right)$ still denoted by $\gamma_{x}$. Using this map we have a map $x \otimes \gamma_{x}$ from $\Lambda^{-}\left(X_{s}\right) \otimes \Phi\left(D_{s}\right)$ to $\Lambda^{-}\left(X_{s^{\prime}}\right) \otimes \Phi\left(D_{s^{\prime}}\right)$ defined by:

$$
u \otimes v \mapsto x \wedge u \otimes \gamma_{x}(v)
$$

Notice that this map is trivial on $\Lambda^{-}\left(X_{s}\right) \otimes \Phi\left(D_{s}\right)$ if $x$ belongs to $X_{s}$.

It is easy to see that the map $d=\sum_{x} x \otimes \gamma_{x}$ is a differential on $E$ of degree -1 . So we get a complex $(E, d)$ denoted by $k h(D)$ (or $k h(D, R))$.

2.3 Remark: If $R=\mathbf{Z}[\alpha] /\left(\alpha^{2}\right)$ and $D$ is oriented, the classical Khovanov complex of $D$ is essentially isomorphic to some suspension of $k h(D)$.

\subsection{The operators $T_{p}$.}

If $D$ be a link diagram, a point in $D$ which is not a crossing will be called a regular point in $D$.

Let $D$ be a link diagram and $p$ be a regular point in $D$. Let $a$ be an element of $R$. If $s$ is a state on $D$, there is a unique component $C$ of $D_{s}$ containing $p$. Denote by $D^{\prime}$ the complement of $C$ in $D_{s}$. The multiplication by $a \otimes 1$ in $\Phi\left(D_{s}\right)=\Phi(C) \otimes \Phi\left(D^{\prime}\right)=$ $R \otimes \Phi\left(D^{\prime}\right)$ is an endomorphism $f$ of $\Phi\left(D_{s}\right)$. So the map:

$$
1 \otimes f: u \otimes v \mapsto u \otimes f(v)
$$


is an endomorphism of $k h(D)$. This endomorphism will be denoted by $T_{p}(a)$.

2.5 Proposition: Let $D$ be a link diagram. Then, for every regular point $p \in D$ and every $a \in R, T_{p}(a)$ is a morphism of complexes of degree 0 from $k h(D)$ to itself. Moreover these operators satisfy the following properties:

- The operators $T_{p}(a)$ commute.

- The map $a \mapsto T_{p}(a)$ is a $K$-algebra homomorphism from $R$ to $\operatorname{End}(k h(D))$.

- Let $p$ and $q$ be two regular points in $D$. Suppose that these points are the endpoints of a path in $D$ going through exactly one crossing. Then, for every $a \in R$ the two operators $T_{p}(a)$ and $T_{q}(\bar{a})$ are homotopic.

Proof: All these properties are easy to check except the last one.

Denote by $x$ the crossing between $p$ and $q$.

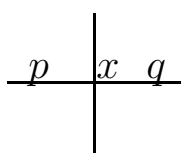

Denote by $D^{+}$(resp. $D^{-}$) the diagram obtained from $D$ by a positive (resp. negative) resolution at $x$. By setting $U=k h\left(D^{+}\right)$and $V=k h\left(D^{-}\right)$, we have:

$$
k h(D)=1 \otimes U \oplus x \otimes V
$$

Denote by $\gamma$ the surgery homomorphism corresponding to the surgery along the path $\gamma_{x}$. It is a morphism from $U$ to $V$ and from $V$ to $U$. Denote by $k$ the map from $k h(D)$ to itself defined by:

$$
k(1 \otimes u)=0 \quad k(x \otimes v)=1 \otimes \gamma(v)
$$

We can check that the corresponding homotopy $d(k)=d \circ k+k \circ d$ is the map $1 \otimes \gamma^{2}$.

Set: $T=T_{p} \otimes T_{q}$. This operator is a map from $R \otimes R$ to the algebra of endomorphisms of $k h(D)$. It is easy to check the following:

$d(k)=T(\Delta(1))=T_{p}(\omega) T_{q}(\alpha)-T_{p}(\omega \bar{\alpha})=T_{q}(\omega) T_{p}(\alpha)-T_{q}(\omega \bar{\alpha})=T_{q}(\omega)\left(T_{p}(\alpha)-T_{q}(\bar{\alpha})\right)$

and $T_{p}(\alpha)$ is homotopic to $T_{q}(\bar{\alpha})$ because $\omega$ is invertible. Let $a=u+v \alpha$ be any element in $R$ with $u$ and $v$ in $K$. If $\sim$ is the homotopy relation, we have:

$$
T_{p}(a)=u+v T_{p}(\alpha) \sim u+v T_{q}(\bar{\alpha})=T_{q}(\bar{a})
$$

\subsection{The Khovanov complex $k h(D, p)$.}

Let $D$ be a link diagram and $p$ be a regular point in $D$. Such a pair $(D, p)$ will be called a pointed diagram. The operator $T_{p}$ induces an action of $R$ on the complex $k h(D)$. Using this action $k h(D)$ becomes a graded differential $R$-module denoted by $k h(D, p)$ (or $k h(D, p, R)$ ). It is easy to see that $k h(D, p)$ is free over $R$.

The algebra $R$ may be big: the transcendance degree of $R_{0}$ is 4 . Nevertheless the complex $k h(D, p)$ can be reduced to a smaller complex. 
Consider a graded commutative ring $\Lambda$. Denote by $\mathscr{M}_{* *}(\Lambda)$ the class of bigraded differential free $\Lambda$-modules $C$ satisfying the following:

$$
\partial^{\circ} a=n, \quad \partial^{\circ} u=(p, q) \quad \Longrightarrow \quad \partial^{\circ}(a u)=(p, q+n), \quad \partial^{\circ}(d u)=(p-1, q-1)
$$

for every $a \in \Lambda$ and $u \in C$. The first component of this degree is called the homological degree and the second the $q$-degree.

Consider the ring $A=\mathbf{Z}\left[t, \delta,(1-t \delta)^{-1}\right]$. This ring is graded by the rule:

$$
\partial^{\circ} t=2 \quad \partial^{\circ} \delta=-2
$$

Moreover $R$ is an $A$-algebra.

2.7 Proposition: There is a correspondance associating to every pointed diagram $(D, p)$ a complex $k h^{\prime}(D, p) \in \mathscr{M}_{* *}(A)$ and an isomorphism:

$$
k h(D, p) \stackrel{\sim}{\longrightarrow} R \underset{A}{\otimes} k h^{\prime}(D, p)
$$

compatible with the degree in $k h(D, p)$ and the homological degree in $k h^{\prime}(D, p)$.

Proof: Let $(D, p)$ be a pointed diagram and $X$ be the set of crossings of $D$. For any state $s: X \rightarrow\{ \pm 1\}$, denote by $C_{s}$ the set of connected components of $D_{s}$. This set is pointed by the component $c_{0}$ of $D_{s}$ containing $p$. Denote by $U_{s}$ the set of maps $\lambda: C_{s} \rightarrow\{ \pm 1\}$ sending $c_{0}$ to 1 . Finally denote by $U$ the set of pairs $(s, \lambda)$, where $s$ is a state and $\lambda$ is an element of $U_{s}$.

Because of the universality of $R_{0}$, we may as well suppose that $R$ is the algebra $R_{0}$. In this case, we have a degree in $R$ :

$$
\partial^{\circ} \alpha=\partial^{\circ} \bar{\alpha}=-2 \quad \partial^{\circ} \omega=\partial^{\circ} \bar{\omega}=0 \quad \Longrightarrow \quad \partial^{\circ} t=2 \quad \partial^{\circ} \delta=-2
$$

For every state $s$, set:

$$
N_{s}=\underset{\lambda \in U_{s}}{\oplus} \operatorname{Re}(s, \lambda) \quad M_{s}=\Lambda^{-}\left(X_{s}\right) \otimes N_{s}
$$

where the elements $e(s, \lambda)$ are formal vectors in one to one correspondance to the elements of $U$. We set also:

$$
M=\oplus_{s} M_{s}
$$

We put a degree on $N_{s}$ by:

$$
\partial^{\circ} a e(s, \lambda)=\partial^{\circ} a+\sum_{c \in C_{s}} \lambda(c)
$$

and a bidegree on $M$ by:

$$
\partial^{\circ}(u \otimes v)=\left(\partial^{\circ} u, \partial^{\circ} v\right)
$$

Let $s$ be a state. Take a numbering of $C_{s}: C_{s}=\left\{c_{0}, c_{1}, \ldots, c_{q-1}\right\}$ in such a way that $c_{0}$ contains the point $p$. Every $\lambda \in U_{s}$ is on the form: $c_{i} \mapsto \lambda_{i}$ with $\lambda_{0}=1$. 
Consider elements $a_{i} \in R$ for $0 \leq i<q$. For every $\lambda \in U_{s}$ we set:

$$
a_{i}(\lambda)=\left\{\begin{array}{cc}
a_{i} & \text { if } \lambda_{i}=1 \\
\varepsilon\left(a_{i}\right) & \text { if } \lambda_{i}=-1
\end{array}\right.
$$

Then we get a map $\varphi_{s}$ from $\Phi\left(D_{s}\right)=R^{\otimes C_{s}}$ to $N_{s}$ defined by:

$$
\otimes_{i} a_{i} \mapsto \sum_{\lambda \in U_{s}}\left(\prod_{i} a_{i}(\lambda)\right) e(s, \lambda)
$$

It is easy to check that this map is $R$-linear and bijective. Its inverse is given by:

$$
\varphi_{s}^{-1}(e(s, \lambda))=\prod_{0 \leq i<q} b_{i}
$$

with:

$$
b_{i}=\left\{\begin{array}{cc}
\omega_{i} \omega_{0}^{-1} & \text { if } \lambda_{i}=1 \\
\omega_{i}\left(\alpha_{i}-\alpha_{0}\right) & \text { if } \lambda_{i}=-1
\end{array}\right.
$$

and: $u_{i}=1^{\otimes i} \otimes u \otimes 1^{\otimes(q-i-1)}$ for $u=\omega$ or $u=\alpha$.

The isomorphisms $\varphi_{s}$ induce an isomorphism $\varphi$ from $k h(D, p)$ to $M$. Via this isomorphism, the differential on $k h(D, p)$ induces a differential $d^{\prime}$ on $M$. A straightforward computation shows that the bidegree of $d^{\prime}$ is $(-1,-1)$ and the entries of the matrix associated to $d^{\prime}$ are in $\left\{0, \pm 1, \pm \delta, \pm \theta^{-1}, \pm \theta^{-1} t, \pm \theta^{-1} \delta\right\}$. That implies the result with:

$$
k h^{\prime}(D, p)=\oplus_{s} \Lambda^{-}\left(X_{s}\right) \otimes\left(\underset{\lambda \in U_{s}}{\oplus} A e(s, \lambda)\right)
$$

Using technics in [Kh4] it is possible to get a stronger reduction. Let $\beta$ be the element $\alpha-\bar{\alpha}=\delta / \omega \in R_{0}$. Every Frobenius algebra of rank 2 is a $\mathbf{Z}[\beta]$-algebra. Moreover the map $t \mapsto 0$ and $\delta \mapsto \beta$ induces a ring homomorphism $A \rightarrow \mathbf{Z}[\beta]$ and $\mathbf{Z}[\beta]$ is an $A$-algebra.

2.8 Proposition: For each pointed diagram $(D, p)$, denote by $k h^{\prime \prime}(D, p)$ the complex $\mathbf{Z}[\beta] \otimes_{A} k h^{\prime}(D, p) \in \mathscr{M}_{* *}(\mathbf{Z}[\beta])$.

Then, for each pointed diagram $(D, p)$, there is an isomorphism of complexes:

$$
k h(D, p) \stackrel{\sim}{\longrightarrow} R \underset{\mathbf{Z}[\beta]}{\otimes} k h^{\prime \prime}(D, p)
$$

compatible with the degree in $k h(D, p)$ and the homological degree in $k h^{\prime \prime}(D, p)$.

Proof: Let $R^{\prime}$ be the algebra $R$ equipped with the following coproduct and counit:

$$
\Delta^{\prime}(u)=\Delta(u / \omega) \quad \varepsilon^{\prime}(u)=\varepsilon(\omega u)
$$

It is easy to see that $R^{\prime}$ is a Frobenius algebra with generator $\alpha$ and twisting element 1. Then $R^{\prime}$ is an $A$-algebra and $t$ and $\delta$ in $A$ are sent to 0 and $\beta$ in $R$. So the $A$-algebra structure of $R^{\prime}$ is actually an $\mathbf{Z}[\beta]$-algebra structure. 
Let $(D, p)$ be a pointed diagram. For every state $s, D_{s}$ is oriented. In particular $D_{1}$ (corresponding to the state $x \mapsto 1$ ) is the oriented boundary of a unique compact $K$ in the plane. Consider a point $q$ near $p$ in the interior of $K$.

Let $s$ be a state. If $c$ is a component of $D_{s}, c$ is the oriented boundary of a unique compact $K_{s}(c)$ in $S^{2}$. So we define an integer $f(s, c)$ by:

$$
f(s, c)=\left\{\begin{array}{cc}
\chi\left(K_{s}(c) \cap K\right)+1-\chi(K) & \text { if } q \in K_{s}(c) \\
\chi\left(K_{s}(c) \cap K\right) & \text { otherwise }
\end{array}\right.
$$

where $\chi$ is the Euler characteristic.

It is easy to see the following:

Let $s$ be a state, and $x$ be a crossing of $D$ with $s(x)=1$. Denote by $s^{\prime}$ the state $s$ modified at $x\left(s^{\prime}=s e_{x}\right)$. If the surgery operator $\gamma_{x}$ connects two components $c$ and $c^{\prime}$ of $D_{s}$ into a component $c^{\prime \prime}$ of $D_{s^{\prime}}$, we have:

$$
f\left(s^{\prime}, c^{\prime \prime}\right)=f(s, c)+f\left(s, c^{\prime}\right)
$$

If the surgery operator disconnects a component $c$ of $D_{s}$ into two components $c^{\prime}$ and $c^{\prime \prime}$ in $D_{s^{\prime}}$, we have:

$$
f(s, c)+1=f\left(s^{\prime}, c^{\prime}\right)+f\left(s^{\prime}, c^{\prime \prime}\right)
$$

So, following [Kh4], we get an isomorphism between the complexes $k h(D, p, R)$ and $k h\left(D, p, R^{\prime}\right)$. This isomorphism is defined as follows:

$$
u \otimes\left(\otimes_{c} v_{c}\right) \mapsto u \otimes\left(\otimes_{c} \frac{v_{c}}{\omega^{f(s, c)}}\right)
$$

for $u \in \Lambda^{-}\left(X_{s}\right)$ and $v_{c} \in R$. Thus we have:

$$
\begin{aligned}
& k h(D, p, R) \simeq k h\left(D, p, R^{\prime}\right) \simeq R^{\prime} \underset{A}{\otimes} k h^{\prime}(D, p) \\
& \simeq R^{\prime} \underset{\mathbf{Z}[\beta]}{\otimes} \mathbf{Z}[\beta] \underset{A}{\otimes} k h^{\prime}(D, p)=R^{\prime} \underset{\mathbf{Z}[\beta]}{\otimes} k h^{\prime \prime}(D, p)
\end{aligned}
$$

The only problem with this new reduction is the fact that $\beta$ is not necessarily stable under the endomorphisms of $R$.

Another (more serious) problem is the fact that these reductions do not induce any canonical reduction for $k h(D)$.

\subsection{The operators $\widehat{T}_{p}$.}

If a link diagram $D$ is oriented, it is possible to modify the operators $T_{p}$ in the following way:

Suppose $p$ is a regular point in $D$. Let $q$ be a point in a neighborough of $p$ and on the left hand side of $D$ and $n$ be the winding number of $D$ about $q$. For every $a \in R$, define $\widehat{T}_{p}(a)$ as the operator $T_{p}(a)$ if $n$ is even and the operator $T_{p}(\bar{a})$ if $n$ is odd. So $\widehat{T}_{p}$ acts on $k h(D)$. It is easy to see that all these operators commute and that the homotopy class of $\widehat{T}_{p}(a)$ depends only on $a$ and the component of $D$ containing $p$. 


\subsection{Khovanov complexes of oriented diagrams.}

Let $D$ be an oriented link diagram. Since $D$ is oriented, each crossing of $D$ has a sign. Denote by $X_{-}$the set of negative crossings of $D$. So we define:

$$
K H(D)=k h(D) \otimes \Lambda^{+}\left(X_{-}\right)
$$

This is a graded differential $K$-module. If needed this complex will be denoted by $K H(D, R)$.

The main result of this paper is to prove that the correspondance $D \mapsto K H(D)$ comes from a monoidal functor from the category of cobordisms of oriented links in $\mathbf{R}^{3}$ to the homotopy category of $K$-complexes.

If $R=\mathbf{Z}[\alpha] / \alpha^{2}, K H(D)$ is essentially isomorphic to the classical Khovanov complex. But the isomorphism between these two complexes is not canonical. It is canonical only up to sign.

If $p$ is a regular point in $D$, the operators $\widehat{T}_{p}(a)$ induce a structure of $R$-complex on $K H(D)$. This complex will be denoted by $K H(D, p)$ (or $K H(D, p, R))$.

We have also the complex $K H^{\prime}(D, p)$ : In the case: $\widehat{T}_{p}(a)=T_{p}(a)$ the complex $K H^{\prime}(D, p)$ is the complex $k h^{\prime}(D, p) \otimes \Lambda^{+}\left(X_{-}\right)$. In the case: $\widehat{T}_{p}(a)=T_{p}(\bar{a}), K H^{\prime}(D, p)$ is the complex $k h^{\prime}\left(D^{\prime}, p\right) \otimes \Lambda^{+}\left(X_{-}\right)$where the $D^{\prime}$ is the diagram $D$ with the opposite orientation. In any case $K H^{\prime}(D, p)$ is a complex in $\mathscr{M}_{* *}(A)$ and $K H(D, p)$ is isomorphic to $R \otimes_{A} K H^{\prime}(D, p)$.

As before we have also the complex $K H^{\prime \prime}(D, p)=\mathbf{Z}[\beta] \otimes_{A} K H^{\prime}(D, p)$ in $\mathscr{M}_{* *}(\mathbf{Z}[\beta])$.

2.11 Proposition: Let $D$ be an oriented link diagram. Consider a circle $C$ contained in a half plane disjoint from $D$ and oriented clockwise. Denote by $\left(D^{\circ}, p\right)$ the union $D \cup C$ pointed by a point $p$ in $C$. Then we have canonical isomorphisms of $R$-complexes:

$$
R \underset{K}{\otimes} K H(D) \simeq R \underset{A}{\otimes} K H^{\prime}\left(D^{\circ}, p\right) \simeq R \underset{\mathbf{Z}[\beta]}{\otimes} K H^{\prime \prime}\left(D^{\circ}, p\right)
$$

Proof: The $R$-complex $R \otimes K H(D)$ is obviously isomorphic to $K H\left(D^{\circ}, p, R\right)$. The result follows.

\subsection{The modules $E(D)$ and $E(D, p)$}

Consider an oriented link diagram $D$. Denote by $C$ the set of components of $D$, by $X$ the set of crossings of $D$ and by $e: X \rightarrow\{ \pm\}$ the map sending each crossing to its sign. For each map $\sigma: C \rightarrow\{ \pm\}$ and each sign $e$ denote by $D_{\sigma}^{e}$ the subdiagram of $D$ where $\sigma$ is equal to $e$. Denote also by $D(\sigma)$ the diagram $D$ where the orientation on $D_{\sigma}^{-}$is changed and by $Y(\sigma) \subset X$ the set of crossings between $D_{\sigma}^{+}$and $D_{\sigma}^{-}$.

For any ring $B$ denote by $E(D, B)$ the following graded $B$-module:

$$
E(D, B)=\underset{\sigma: C \rightarrow\{ \pm\}}{\oplus} \Lambda^{-e}(Y(\sigma)) \otimes B v(\sigma)
$$


where the $v(\sigma)$ 's are formal vectors in one to one correspondance with the $\sigma$ 's.

Let $p$ be a regular point in $D$. Let $e_{0}$ be the sign with is equal to + if and only if the operators $T_{p}$ and $\widehat{T}_{p}$ are the same on $K H(D)$. The set $C$ is pointed by the component $c_{0}$ containing $p$. Denote by $\widehat{C}$ the set of maps $\sigma: C \rightarrow\{ \pm\}$ sending $c_{0}$ to $e_{0}$. So, for any ring $B$ denote by $E(D, p, B)$ the following graded $B$-module:

$$
E(D, p, B)=\bigoplus_{\sigma \in \widehat{C}} \Lambda^{-e}(Y(\sigma)) \otimes B v(\sigma)
$$

If $B$ is a graded ring, $E(D, B)$ and $E(D, p, B)$ is bigraded by the rule:

$$
\partial^{\circ}(u \otimes b v(\sigma))=\left(\partial^{\circ} u, \partial^{\circ} b+b_{\sigma}\right)
$$

where $b_{\sigma}$ the number of components of the oriented resolution of $D(\sigma)$. By equipping $E(D, B)$ and $E(D, p, B)$ with the zero differential, these modules become $B$ complexes or complexes in $\mathscr{M}_{* *}(B)$.

2.13 Lemma: Let $(D, p)$ be a pointed oriented diagram. Then there are caconical morphisms of complexes:

$$
\begin{gathered}
\varphi(R): K H(D, p, R) \longrightarrow E(D, p, R) \\
\varphi^{\prime}: K H^{\prime}(D, p) \longrightarrow E(D, p, A) \\
\varphi^{\prime \prime}: K H^{\prime \prime}(D, p) \longrightarrow E(D, p, \mathbf{Z}[\beta])
\end{gathered}
$$

of degree 0 or $(0,0)$.

Proof: Let $\sigma$ be an element of $\widehat{C}$. The oriented resolution of $D(\sigma)$ (i.e. the only resolution compatible with the orientation) is the diagram $D_{s}$ where $s$ is the state $x \mapsto e(x) \sigma(c) \sigma\left(c^{\prime}\right)$ and $c$ and $c^{\prime}$ the components of $D$ containing $x$.

Let $Z(\sigma)$ be the complement of $Y(\sigma)$ in $X$. For each subset $H$ of $X$ we denote by $H_{+}$(resp. $H_{-}$) the set of positive crossings (resp. negative crossings) in $H$. So we have:

$$
X_{s}=Y(\sigma)_{+} \cup Z(\sigma)_{-} \quad X_{-}=Y(\sigma)_{-} \cup Z(\sigma)_{-}
$$

For any $u \in R$ and any sign $e$ define the element $u^{(e)}$ by:

$$
u^{(+)}=u \quad u^{(-)}=\bar{u}
$$

The set $C_{s}$ of components of $D_{s}$ has $q=b_{\sigma}$ elements. Take a numbering of $C_{s}$ : $C_{s}=\left(c_{0}(s), c_{1}(s), \ldots, c_{q-1}(s)\right)$, such that $c_{0}(s)$ contains the point $p$. For each $i<q$, denote by $d_{i}$ the winding number of $D_{s}$ about a point to the left of $c_{i}(s)$ and by $a_{i}$ the sign $(-1)^{d_{i}}$.

We have a morphism $f_{\sigma}$ from $K H(D)$ to $\Lambda^{-e}(Y(\sigma)) \otimes R$ :

This morphism is trivial on $\Lambda^{-}\left(X_{s^{\prime}}\right) \otimes \Phi\left(D_{s^{\prime}}\right) \otimes \Lambda^{+}\left(X_{-}\right)$for $s^{\prime} \neq s$. For $s^{\prime}=s$, we have:

$\Lambda^{-}\left(X_{s}\right) \otimes \Phi\left(D_{s}\right) \otimes \Lambda^{+}\left(X_{-}\right) \simeq \Lambda^{-}\left(Y(\sigma)_{+}\right) \otimes \Lambda^{-}\left(Z(\sigma)_{-}\right) \otimes R^{\otimes q} \otimes \Lambda^{+}\left(Y(\sigma)_{-}\right) \otimes \Lambda^{+}\left(Z(\sigma)_{-}\right)$ 


$$
\simeq \Lambda^{-e}(Y(\sigma)) \otimes R^{\otimes q}
$$

and $\Lambda^{-}\left(X_{s}\right) \otimes \Phi\left(D_{s}\right) \otimes \Lambda^{+}\left(X_{-}\right)$is canonically isomorphic to $\Lambda^{-e}(Y(\sigma)) \otimes R^{\otimes q}$. So the map $f_{\sigma}$ is on $\Lambda^{-}\left(X_{s}\right) \otimes \Phi\left(D_{s}\right) \otimes \Lambda^{+}\left(X_{-}\right)$the following:

$$
\Lambda^{-}\left(X_{s}\right) \otimes \Phi\left(D_{s}\right) \otimes \Lambda^{+}\left(X_{-}\right) \stackrel{\sim}{\longrightarrow} \Lambda^{-e}(Y(\sigma)) \otimes R^{\otimes q} \stackrel{1 \otimes g}{\longrightarrow} \Lambda^{-e}(Y(\sigma)) \otimes R
$$

where $g$ is the map:

$$
b_{0} \otimes b_{1} \otimes \ldots \otimes b_{q-1} \mapsto \prod_{i} b_{i}^{\left(a_{i}\right)}
$$

More precisely, $f_{\sigma}$ is the map:

$$
u_{1} \otimes u_{2} \otimes u_{3} \otimes u_{4} \otimes u_{5} \mapsto \quad(-1)^{\left|u_{2}\right|\left|u_{4}\right|}<u_{2}, u_{5}>u_{1} \wedge u_{4} \otimes g^{\prime}\left(u_{3}\right)
$$

for every $\left(u_{1}, u_{2}, u_{3}, u_{4}, u_{5}\right) \in \Lambda^{-}\left(Y(\sigma)_{+}\right) \times \Lambda^{-}\left(Z(\sigma)_{-}\right) \times \Phi\left(D_{s}\right) \times \Lambda^{+}\left(Y(\sigma)_{-}\right) \times$ $\Lambda^{+}\left(Z(\sigma)_{-}\right)$, where $|u|$ is the degree of $u, g^{\prime}$ is the map:

$$
\Phi\left(D_{s}\right) \stackrel{\sim}{\longrightarrow} R^{\otimes q} \stackrel{g}{\longrightarrow} R
$$

and $<$ ?, ? $>$ is the isomorphism $\Lambda^{-}\left(Z(\sigma)_{-}\right) \otimes \Lambda^{+}\left(Z(\sigma)_{-}\right) \stackrel{\sim}{\longrightarrow} \mathbf{Z}$ defined by:

$$
<x_{1} \wedge x_{2} \wedge \ldots \wedge x_{n}, x_{1} \wedge x_{2} \wedge \ldots \wedge x_{n}>=(-1)^{n(n-1) / 2}
$$

It is easy to see that this map is a morphism of degree 0 from $K H(D)$ to $\Lambda^{-e}(Y(\sigma)) \otimes R$ inducing a morphism $\varphi_{\sigma}(R)$ from $K H(D, p, R)$ to $\Lambda^{-e}(Y(\sigma)) \otimes R v(\sigma)$.

On the level of complexes $K H^{\prime}$, we get a morphism $\varphi_{\sigma}$ from $K H^{\prime}(D, p)$ to $\Lambda^{-e}(Y(\sigma)) \otimes A v(\sigma)$. This morphism sends $e(s, \lambda)$ to 0 if there exists an $i$ with $a_{i}=e_{0}$ and $\lambda_{i}=-$. If it is not the case, the morphism sends $e(s, \lambda)$ to $\theta^{-c}(-\delta)^{d} v\left(D^{\prime}\right)$, where $d$ is the number of $i$ such that: $\lambda_{i}=-$ and $c$ the number of $i$ such that: $a_{i}=-e_{0}$. Because of the degree of $v(\sigma)$, the bidegree of $\varphi_{\sigma}$ is $(0,0)$.

By taking the sum of all these morphisms we get the desired morphisms $\varphi(R), \varphi^{\prime}$ and then $\varphi^{\prime \prime}$.

2.14 Remark: Suppose $\delta$ is invertible in $R$. Then the map $\varphi(R)$ has a section. By using the notations above this section is a morphism of complexes defined by:

$\Lambda^{-e}(Y(\sigma)) \otimes R v(\sigma) \stackrel{\sim}{\longrightarrow} \Lambda^{-}\left(X_{s}\right) \otimes R v(\sigma) \otimes \Lambda^{+}\left(X_{-}\right) \stackrel{1 \otimes g \otimes 1}{\longrightarrow} \Lambda^{-}\left(X_{s}\right) \otimes \Phi\left(D_{s}\right) \otimes \Lambda^{+}\left(X_{-}\right)$

where $g$ is the map:

$$
u v(\sigma) \mapsto u_{0} \prod_{0 \leq i<j<q}\left(\delta_{0}^{-1}\left(\omega_{i}^{\left(a_{i}\right)} \alpha_{j}^{\left(a_{j}\right)}-\left(\omega_{i} \bar{\alpha}_{i}\right)^{\left(a_{i}\right)}\right)\right)
$$

and $u_{i}$ is, for every $u \in R$, the element $1^{\otimes i} \otimes u \otimes 1^{\otimes(q-i-1)}$.

2.15 Theorem: Let $(D, p)$ be an pointed oriented link diagram. Suppose that the underlying graph of $D$ is connected. Then the morphisms:

$$
\varphi(R): K H(D, p, R) \longrightarrow E(D, p, R)
$$




$$
\begin{gathered}
\varphi^{\prime}: K H^{\prime}(D, p) \longrightarrow E(D, p, A) \\
\varphi^{\prime \prime}: K H^{\prime \prime}(D, p) \longrightarrow E(D, p, \mathbf{Z}[\beta])
\end{gathered}
$$

are surjective and the action of $\beta$ is homotopically nilpotent on their kernels.

Proof: Because the underlying graph of $D$ is connected, the map sending $\sigma \in \widehat{C}$ to the state $s: x \mapsto e(x) \sigma(c) \sigma\left(c^{\prime}\right)$ is injective. Therefore all maps $\varphi$ are surjective, in particular $\varphi^{\prime \prime}$.

The last thing to do is to prove that the action of $\beta$ on the kernel $U$ of $\varphi^{\prime \prime}$ is homotopically nilpotent. But that is equivalent to the fact that $\mathbf{Z}\left[\beta^{ \pm}\right] \otimes \mathbf{Z}[\beta] U$ is acyclic. Let $B$ be the ring $Z\left[\beta^{ \pm}\right]$and $R_{1}$ be the algebra $B \times B$. Using the diagonal map $B \rightarrow B \times B, R_{1}$ is a Frobenius $B$-algebra of rank 2 with generator $(1,0)$. In this algebra, the involution is: $(u, v) \mapsto(v, u)$, the twisting element is 1 and the counit is: $(u, v) \mapsto u-v$.

It is clear that $B \otimes U$ is acyclic if and only if $R_{1} \otimes U$ is acyclic. Therefore it is enough to prove that $\varphi\left(R_{1}\right)$ induces an isomorphism in homology from $K H\left(D, p, R_{1}\right)$ to $E\left(D, p, R_{1}\right)$. But in $R_{1}$ we have two orthogonal idempotents:

$$
\pi_{+}=(1,0) \quad \pi_{-}=(0,1)
$$

So we can use the Karoubi completion method of Bar-Natan and Morrison [BM] to prove that $\varphi\left(R_{1}\right)$ is a homology equivalence.

2.16 Remark: If the underlying graph of $D$ is not connected the result is still true but the kernels have to be replaced by the homotopy kernels. In particular the morphisms $\varphi$ are homotopy equivalences if $\delta$ is invertible in $R$.

\section{Elementary moves.}

Consider an elementary move (i.e. a Reidemeister move or a surgery move) $f$ : $D \rightarrow D^{\prime}$ transforming a link diagram $D$ into a link diagram $D^{\prime}$. If the correspondance $D \mapsto k h(D)$ comes from a functor on the category of cobordisms of links, the move $f$ would have to induce a morphism $f_{*}$ from $k h(D)$ to $k h\left(D^{\prime}\right)$. So the first thing to do is to associate to every elementary move $f: D \rightarrow D^{\prime}$ a morphism from $k h(D)$ to $k h\left(D^{\prime}\right)$.

3.1 Proposition: There is a correspondance associating to every elementary move $f: D \rightarrow D^{\prime}$ a morphism $f^{0}: k h(D) \rightarrow k h\left(D^{\prime}\right)$ such that:

if $f$ is a Reidemeister move with inverse move $g, f^{0}$ is a homotopy equivalence and $g^{0}$ is a homotopy inverse of $f$.

Proof: In order to construct the correspondance $f \mapsto f^{0}$, we have to consider all types of elementary moves.

Surgery moves: There is three kinds of surgery moves: surgery moves of index 0,1 or 2. A surgery move $f: D \rightarrow D^{\prime}$ of index 0 transforms $D$ by adding a circle bounding 
a disk in the plane which is disjoint from $D$ and the inverse move $g: D^{\prime} \rightarrow D$ is a surgery move of index 2 .

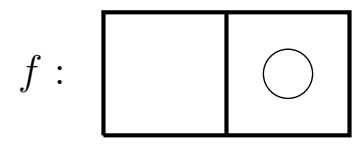

Let $D$ be a link diagram. Consider a path $\gamma$ embedded in the plane which doesn't meet any crossing point of $D$ and intersects $D$ in its boundary. Such a path is called a surgery path of $D$.

A surgery move $f: D \rightarrow D^{\prime}$ of index 1 is a modification $D \mapsto D^{\prime}$ by surgery along some surgery path $\gamma$ of $D$.

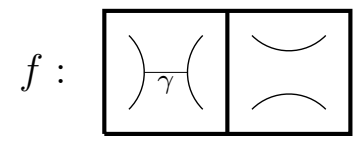

Suppose $f: D \rightarrow D^{\prime}$ is a surgery move of index 0 and $g: D^{\prime} \rightarrow D$ its inverse move. Then we have: $k h\left(D^{\prime}\right)=k h(D) \otimes R$ and the map $f^{0}$ and $g^{0}$ are defined by:

$$
\forall(u, a) \in k h(D) \times R, \quad f^{0}(u)=u \otimes 1, \quad g^{0}(u \otimes a)=u \varepsilon(a)
$$

Suppose $f: D \rightarrow D^{\prime}$ is a surgery move of index 1 . This surgery move is defined by a surgery path $\gamma$. For every state $s$ the surgery induces, via the functor $\Phi$, a map $\gamma: \Phi\left(D_{s}\right) \rightarrow \Phi\left(D_{s}^{\prime}\right)$ and these maps induce a map $f^{0}$ from $k h(D)$ to $k h\left(D^{\prime}\right)$. It is easy to see that $f^{0}$ is a morphism of complexes.

Reidemeister moves of type I: There is four kinds of Reidemeister moves of type I depending of two signs. The first sign is + (or 1 ) if the move creates a new crossing and $-($ or -1$)$ if it removes one crossing. The second one is the sign of this crossing (for any orientation of the diagram). We say that $f$ is a Reidemeister move of type $\mathrm{I}_{e^{\prime}}^{e}$ if $f$ is a Reidemeister move where $e$ is the first sign and $e^{\prime}$ the second one. It is clear that the inverse move of a Reidemeister move of type $\mathrm{I}_{e^{\prime}}^{e}$ is a Reidemeister move of type $\mathrm{I}_{e^{\prime}}^{-e}$.

Consider a Reidemeister move $f: D \rightarrow D^{\prime}$ of type $\mathrm{I}_{e}^{+}$and denote by $g$ its inverse move (of type $\mathrm{I}_{e}^{-}$). Let $x$ be the created crossing and $U$ be the complex $k h(D)$. Let $p$ be a point in $D$ which is near $x$. So we have:

$$
k h\left(D^{\prime}\right)= \begin{cases}U \otimes R \oplus x \otimes U & \text { if } e=+ \\ U \oplus x \otimes U \otimes R & \text { if } e=-\end{cases}
$$

Suppose $e=+$. Then the move is the following:

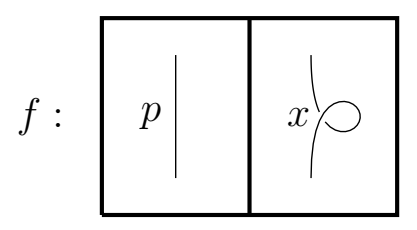

and we set:

$\forall(u, a) \in U \times R, \quad f^{0}(u)=u \otimes \omega \alpha-T_{p}(\alpha) u \otimes \omega, \quad g^{0}(x \otimes u)=0, \quad g^{0}(u \otimes a)=u \varepsilon(a)$ 
Suppose $e=-$. Then the move is the following:

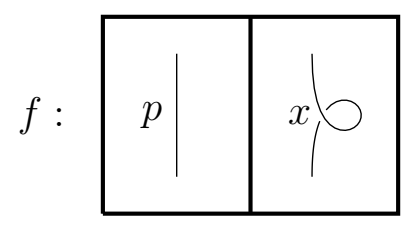

and we set:

$$
\forall(u, a) \in U \times R, \quad f^{0}(u)=x \otimes u \otimes 1 \quad g^{0}(u)=0, \quad g^{0}(x \otimes u \otimes a)=T_{p}(\bar{a}) u
$$

It is not difficult to see that $f^{0}$ and $g^{0}$ are morphisms of complexes and that $g^{0}$ is a left inverse of $f^{0}$. Moreover the cokernel of $f^{0}$ is the mapping cone of an isomorphism. So $f^{0}$ is a homotopy equivalence and $g^{0}$ is a homotopy inverse of $f^{0}$.

Reidemeister moves of type II: There is two kinds of Reidemeister moves of type II: Reidemeister moves of type $\mathrm{II}^{+}$that create two new crossings and their inverses of type $\mathrm{II}^{-}$.

Consider a Reidemeister move $f: D \rightarrow D^{\prime}$ of type $\mathrm{II}^{+}$and its inverse move $g: D^{\prime} \rightarrow D$. Let $D^{\prime \prime}$ be the diagram obtained from $D$ by a surgery along a path joining the two branches of $D$ modified by $f$.

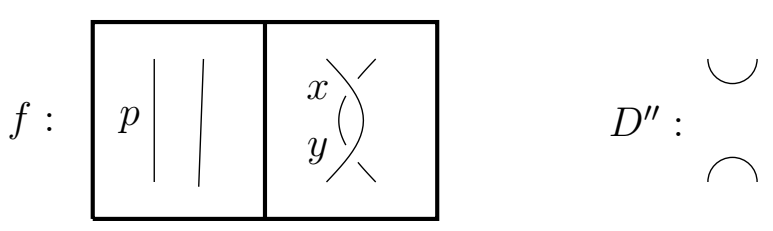

Denote by $U$ and $V$ the complexes $k h(D)$ and $k h\left(D^{\prime \prime}\right)$. Then we have:

$$
k h\left(D^{\prime}\right)=V \oplus x \otimes U \oplus y \otimes V \otimes R \oplus x \wedge y \otimes V
$$

where $x$ and $y$ are the created crossings in $D^{\prime}$. Surgery moves from $D$ to $D^{\prime \prime}$ and from $D^{\prime \prime}$ to $D$ induce morphisms $\gamma: U \rightarrow V$ and $\gamma: V \rightarrow U$. The morphisms $f^{0}$ and $g^{0}$ are defined by:

$$
\begin{gathered}
\forall u \in U, \quad f^{0}(u)=x \otimes u+y \otimes \gamma\left(T\left(\omega^{-1}\right) u\right) \otimes \omega, \quad g^{0}(x \otimes u)=u \\
\forall(v, a) \in V \times R, \quad g^{0}(v)=g^{0}(x \wedge y \otimes v)=0, \quad g^{0}(y \otimes v \otimes a)=-\varepsilon(a) \gamma(v)
\end{gathered}
$$

where $T$ is the operator $T_{p}$ for some $p$ in one of the two branches of $D$.

We can see that $g^{0}$ doesn't depend on $p$ and $f^{0}$ and $g^{0}$ are morphisms of complexes. Moreover $g^{0}$ is a left inverse of $f^{0}$ and the cokernel of $f^{0}$ is the mapping cone of an isomorphism. So $f^{0}$ is a homotopy equivalence and $g^{0}$ is a homotopy inverse of $f^{0}$.

Reidemeister moves of type III: There is two kinds of Reidemeister moves of type III depending on a sign. If $f: D \rightarrow D^{\prime}$ is a Reidemeister move of type III, three crossings of $D$ are modified. These crossings are the vertices of a triangle $\Theta$ which is oriented by the orientation of the plane. We number the branches starting with the 
top branch and ending with the bottom one. So we get a numbering of the edges of the triangle. We say that the move is of type $\mathrm{III}_{+}$if this numbering is compatible with the orientation of $\Theta$ and III if it is not the case.

Let's denote by $x$ the intersection of the top branch and the bottom branch. The other vertices of $\Theta$ are denoted by $y$ and $z$ in such a way that the numbering $(x, y, z)$ is compatible with the orientation of the triangle. We proceed similarly for the diagram $D^{\prime}$ and the move is the following:

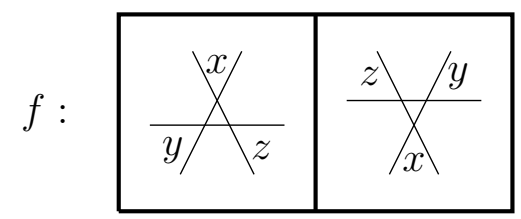

By resolution of $D$ near the triangle we get five new diagrams:

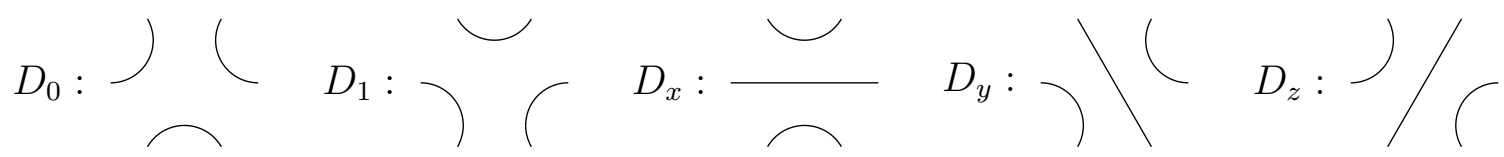

and, for $D^{\prime}$, we get: $D_{0}^{\prime}=D_{1}, D_{1}^{\prime}=D_{0}, D_{x}^{\prime}=D_{x}, D_{y}^{\prime}=D_{y}$ and $D_{z}^{\prime}=D_{z}$.

With these new diagrams we get five new complexes: $U=k h\left(D_{0}\right), V=k h\left(D_{1}\right)$, $A=k h\left(D_{x}\right), B=k h\left(D_{y}\right), C=k h\left(D_{z}\right)$.

Consider six points $p_{i}, i \in \mathbf{Z} / 6$, sitted as follows in $D$ :

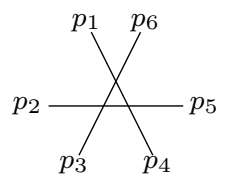

All these points are in a circle $\Gamma$ which bounds a disk $\Delta$. Since the move modifies the diagram $D$ only in $\Delta$, the points $p_{i}$ are also in diagrams $D^{\prime}, D_{0}, D_{1}, D_{x}, D_{y}$ and $D_{z}$.

The arc of $\Gamma$ with endpoints $p_{i}$ and $p_{i-1}$ will be denoted by $\gamma_{i}$. This arc induces a surgery operator still denoted by $\gamma_{i}$. We'll denote also by $T_{i}$ the operator $T_{p_{i}}$. All these operators are well defined on the complexes $U, V, A, B$ and $C$.

Suppose $f$ is a move of type $\mathrm{III}_{+}$. Then the move is the following:

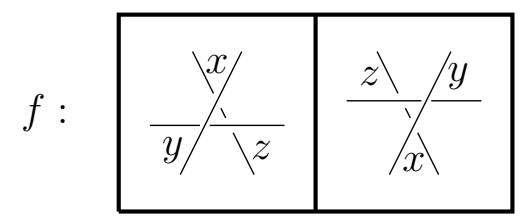

And we have:

$k h(D)=V \oplus x \otimes V \otimes R \oplus y \otimes C \oplus z \otimes B \oplus y \wedge z \otimes U \oplus x \wedge z \otimes V \oplus x \wedge y \otimes V \oplus x \wedge y \wedge z \otimes A$ $k h\left(D^{\prime}\right)=U \oplus x \otimes U \otimes R \oplus y \otimes C \oplus z \otimes B \oplus y \wedge z \otimes V \oplus x \wedge z \otimes U \oplus x \wedge y \otimes U \oplus x \wedge y \wedge z \otimes A$ 
In $k h(D)$ and $k h\left(D^{\prime}\right)$ the differential $d$ is on the form $d=d_{1}+d_{2}$, where $d_{1}$ corresponds to the differentials of the complexes $U, V, A, B, C$. The map $d_{2}$ is defined on $k h(D)$ by the following (for any $r \in R, u \in U, v \in V, a \in A, b \in B, c \in C$ ):

$$
\begin{gathered}
d_{2}(v)=x \otimes \gamma_{1}(v)+y \otimes \gamma_{2}(v)+z \otimes \gamma_{6}(v) \\
d_{2}(x \otimes v \otimes r)=-x \wedge y \otimes T_{3}(r) v-x \wedge z \otimes T_{5}(r) v \\
d_{2}(y \otimes c)=x \wedge y \otimes \gamma_{1}(c)-y \wedge z \otimes \gamma_{4}(c) \\
d_{2}(z \otimes b)=x \wedge z \otimes \gamma_{1}(b)+y \wedge z \otimes \gamma_{4}(b) \\
d_{2}(y \wedge z \otimes u)=x \wedge y \wedge z \otimes \gamma_{1}(u) \\
d_{2}(x \wedge z \otimes v)=-x \wedge y \wedge z \otimes \gamma_{4}(v) \\
d_{2}(x \wedge y \otimes v)=x \wedge y \wedge z \otimes \gamma_{4}(v) \\
d_{2}(x \wedge y \wedge z \otimes a)=0
\end{gathered}
$$

and, by exchanging $U$ and $V$ and replacing $\gamma_{i}$ and $T_{i}$ by $\gamma_{i+3}$ and $T_{i+3}$, we get the differential $d_{2}$ on $k h\left(D^{\prime}\right)$.

So we define the map $f^{0}$ by the following:

$$
\begin{gathered}
f^{0}(v)=0 \\
f^{0}(x \otimes v \otimes r)=\varepsilon(r \omega)\left(x \otimes T_{1}\left(\omega^{-1}\right) \gamma_{2} \gamma_{6}(v) \otimes 1+y \otimes T_{1}\left(\omega^{-1}\right) \gamma_{2}(v)+z \otimes T_{1}\left(\omega^{-1}\right) \gamma_{6}(v)\right) \\
f^{0}(y \otimes c)=-x \otimes \gamma_{4}(c) \otimes 1-y \otimes c \\
f^{0}(z \otimes b)=-z \otimes b \\
f^{0}(x \wedge y \otimes v)=y \wedge z \otimes v \\
f^{0}(x \wedge z \otimes v)=-y \wedge z \otimes v \\
f^{0}(y \wedge z \otimes u)=-x \wedge z \otimes u \\
f^{0}(x \wedge y \wedge z \otimes a)=x \wedge y \wedge z \otimes a
\end{gathered}
$$

We can check that $f^{0}$ is a morphism of complexes of degree 0 . Let $W$ be the $\mathbf{Z}$-module freely generated by $\lambda, d(\lambda), \mu$ and $d(\mu)$. By setting:

$$
\partial^{\circ} \lambda=0 \quad \partial^{\circ} \mu=-1
$$

$W$ becomes an acyclic graded differential Z-module. We have maps $\Psi: W \otimes V \rightarrow$ $k h(D)$ and $\Psi^{\prime}: W \otimes U \rightarrow k h\left(D^{\prime}\right)$ defined by:

$$
\begin{aligned}
\Psi(\lambda \otimes v)=v & \Psi(d(\lambda) \otimes v)=d_{2}(v) \\
\Psi(\mu \otimes v)=x \otimes v \otimes 1 & \Psi(d(\mu) \otimes v)=d_{2}(x \otimes v \otimes 1) \\
\Psi^{\prime}(\lambda \otimes u)=u & \Psi^{\prime}(d(\lambda) \otimes u)=d_{2}(u) \\
\Psi^{\prime}(\mu \otimes u)=x \otimes u \otimes 1 & \Psi^{\prime}(d(\mu) \otimes u)=d_{2}(x \otimes u \otimes 1)
\end{aligned}
$$


It is easy to see that $\Psi$ ans $\Psi^{\prime}$ are injective morphisms of complexes of degree 0 . Since $W$ is acyclic, the images of $\Psi$ and $\Psi^{\prime}$ are acyclic too. Moreover the image of $\Psi$ is killed by $f^{0}$ and the image of $\Psi^{\prime}$ is therefore killed by $g^{0}$, where $g$ is the inverse move of $f$. Modulo these images we have:

$$
\begin{aligned}
& k h(D) \equiv y \otimes C \oplus z \otimes B \oplus y \wedge z \otimes U \oplus x \wedge z \otimes V \oplus x \wedge y \wedge z \otimes A \\
& k h\left(D^{\prime}\right) \equiv y \otimes C \oplus z \otimes B \oplus y \wedge z \otimes V \oplus x \wedge z \otimes U \oplus x \wedge y \wedge z \otimes A
\end{aligned}
$$

and maps $f^{0}$ and $g^{0}$ are on these quotients:

$$
f^{0}:\left\{\begin{array}{rl}
y \otimes c & \mapsto-y \otimes c \\
z \otimes b & \mapsto-z \otimes b \\
y \wedge z \otimes u & \mapsto-x \wedge z \otimes u \\
x \wedge z \otimes v & \mapsto-y \wedge z \otimes v \\
x \wedge y \wedge z \otimes a & \mapsto x \wedge y \wedge z \otimes a
\end{array} \quad g^{0}: \quad\left\{\begin{aligned}
y \otimes c & \mapsto-y \otimes c \\
z \otimes b & \mapsto-z \otimes b \\
y \wedge z \otimes v & \mapsto-x \wedge z \otimes v \\
x \wedge z \otimes u & \mapsto-y \wedge z \otimes u \\
x \wedge y \wedge z \otimes a & \mapsto x \wedge y \wedge z \otimes a
\end{aligned}\right.\right.
$$

Therefore $f^{0}$ and $g^{0}$ are homotopy equivalences and $g^{0}$ is a homotopy inverse of $f^{0}$.

Suppose now the move is of type III_. The move is the following:

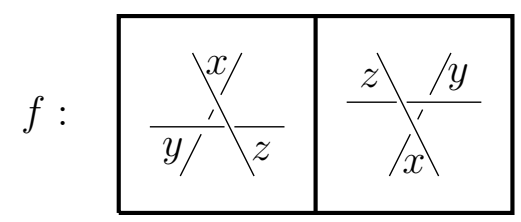

And we have:

$k h(D)=A \oplus x \otimes U \oplus y \otimes V \oplus z \otimes V \oplus y \wedge z \otimes V \otimes R \oplus x \wedge z \otimes C \oplus x \wedge y \otimes B \oplus x \wedge y \wedge z \otimes V$ $k h\left(D^{\prime}\right)=A \oplus x \otimes V \oplus y \otimes U \oplus z \otimes U \oplus y \wedge z \otimes U \otimes R \oplus x \wedge z \otimes C \oplus x \wedge y \otimes B \oplus x \wedge y \wedge z \otimes U$ As before the differentials of $k h(D)$ and $k h\left(D^{\prime}\right)$ are on the form $d=d_{1}+d_{2}$, where $d_{1}$ corresponds to the differentials of the complexes $U, V, A, B, C$. The map $d_{2}$ is defined on $k h(D)$ by the following:

$$
\begin{gathered}
d_{2}(a)=x \otimes \gamma_{2}(a)+y \otimes \gamma_{5}(a)+z \otimes \gamma_{5}(a) \\
d_{2}(x \otimes u)=-x \wedge y \otimes \gamma_{3}(u)-x \wedge z \otimes \gamma_{5}(u) \\
d_{2}(y \otimes v)=x \wedge y \otimes \gamma_{6}(v)-y \wedge z \otimes \gamma_{5}(v) \\
d_{2}(z \otimes v)=x \wedge z \otimes \gamma_{2}(v)+y \wedge z \otimes \gamma_{3}(v) \\
d_{2}(y \wedge z \otimes v \otimes r)=x \wedge y \wedge z \otimes T_{1}(r) v \\
d_{2}(x \wedge y \otimes b)=x \wedge y \wedge z \otimes \gamma_{1}(b) \\
d_{2}(x \wedge z \otimes c)=-x \wedge y \wedge z \otimes \gamma_{1}(c) \\
d_{2}(x \wedge y \wedge z \otimes v)=0
\end{gathered}
$$


and, as before, we get the differential $d_{2}$ on $k h\left(D^{\prime}\right)$ by exchanging $U$ and $V$ and replacing $\gamma_{i}$ and $T_{i}$ by $\gamma_{i+3}$ and $T_{i+3}$. So we define the map $f^{0}$ by the following:

$$
\begin{gathered}
f^{0}(a)=a \\
f^{0}(x \otimes u)=(y+z) \otimes u \\
f^{0}(y \otimes v)=x \otimes v \\
f^{0}(z \otimes v)=0 \\
f^{0}(y \wedge z \otimes v \otimes r)=\varepsilon(r)\left(x \wedge z \otimes \gamma_{2}(v)+y \wedge z \otimes T_{4}\left(\omega^{-1}\right) \gamma_{4} \gamma_{2}(v) \otimes \omega\right) \\
f^{0}(x \wedge y \otimes b)=-x \wedge y \otimes b+y \wedge z \otimes T_{4}\left(\omega^{-1}\right) \gamma_{4}(b) \otimes \omega \\
f^{0}(x \wedge z \otimes c)=-x \wedge z \otimes c-y \wedge z \otimes T_{4}\left(\omega^{-1}\right) \gamma_{4}(c) \otimes \omega \\
f^{0}(x \wedge y \wedge z \otimes v)=0
\end{gathered}
$$

We can check that $f^{0}$ is a morphism of complexes of degree 0 . We have maps $\Psi$ : $W \otimes V \rightarrow k h(D)$ and $\Psi^{\prime}: W \otimes U \rightarrow k h\left(D^{\prime}\right):$

$$
\begin{aligned}
\Psi(\lambda \otimes v)=z \otimes v & \Psi(d(\lambda) \otimes v)=d_{2}(z \otimes v) \\
\Psi(\mu \otimes v)=y \wedge z \otimes v \otimes \omega & \Psi(d(\mu) \otimes v)=d_{2}(y \wedge z \otimes v \otimes \omega) \\
\Psi^{\prime}(\lambda \otimes u)=z \otimes u & \Psi^{\prime}(d(\lambda) \otimes u)=d_{2}(z \otimes u) \\
\Psi^{\prime}(\mu \otimes u)=y \wedge z \otimes u \otimes \omega & \Psi^{\prime}(d(\mu) \otimes u)=d_{2}(y \wedge z \otimes u \otimes \omega)
\end{aligned}
$$

These two maps are injective morphisms of complexes of degree -1 and their images are acyclic subcomplexes killed by $f^{0}$ and $g^{0}$. Modulo these images we have:

$$
\begin{aligned}
& k h(D) \equiv A \oplus x \otimes U \oplus y \otimes V \oplus x \wedge y \otimes B \oplus x \wedge z \otimes C \\
& k h\left(D^{\prime}\right) \equiv A \oplus x \otimes V \oplus y \otimes U \oplus x \wedge y \otimes B \oplus x \wedge z \otimes C
\end{aligned}
$$

and maps $f^{0}$ and $g^{0}$ are:

$$
f^{0}:\left\{\begin{array}{rl}
a & \mapsto a \\
x \otimes u & \mapsto y \otimes u \\
y \otimes v & \mapsto x \otimes v \\
x \wedge y \otimes b & \mapsto-x \wedge y \otimes b \\
x \wedge z \otimes c & \mapsto-x \wedge z \otimes c
\end{array} \quad g^{0}: \quad\left\{\begin{array}{c}
a \mapsto a \\
x \otimes v \mapsto y \otimes v \\
y \otimes u \mapsto x \otimes u \\
x \wedge y \otimes b \mapsto-x \wedge y \otimes b \\
x \wedge z \otimes c \mapsto-x \wedge z \otimes c
\end{array}\right.\right.
$$

Therefore $f^{0}$ and $g^{0}$ are homotopy equivalences and $g^{0}$ is a homotopy inverse of $f^{0}$.

3.2 Remark: For every elementary move $f$, the map $f^{0}$ is a morphism of degree 0 except if $f$ is a Reidemeister move of type $\mathrm{I}_{-}^{e}$ or $\mathrm{II}^{e}$ (with $e= \pm$ ). In these cases $f^{0}$ is a morphism of degree $-e$.

3.3 Elementary moves for oriented link diagrams. Consider an oriented link diagram $D$. Denote by $w$ the function sending each point $x$ outside of $D$ to the winding number of $D$ about $x$. The sign $(-1)^{w(x)}$ will be called the $D$-sign of $x$. If $x$ 
is a crossing of $D$, the function $w$ takes three values $n-1, n, n+1$ near $x$. The sign $(-1)^{n}$ will also be called the $D$-sign of $x$.

In order to describe the orientation of a curve we'll use the following convention: suppose $a$ is a sign. Then the figure

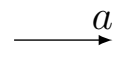

means that the curve is oriented by the arrow if $a=+$ and by the opposite orientation if $a=-$.

Let $f: D \rightarrow D^{\prime}$ be a Reidemeister move of type $\mathrm{I}_{e}^{+}$between oriented diagrams and $g$ be its inverse move. Let $a$ be the winding number of the created loop and $h$ be the $D^{\prime}$-sign of the created crossing. We say that $f$ is a Reidemeister move of type $\mathrm{I}^{+}(e, a, h)$ and that $g$ is a Reidemeister move of type $\mathrm{I}^{-}(e, a, h)$. In this case the diagram $D^{\prime}$ is oriented as follows:

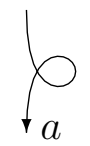

Let $f: D \rightarrow D^{\prime}$ be a Reidemeister move of type $\mathrm{II}^{+}$between oriented diagrams and $g$ be its inverse move. Let $\Gamma$ be the created loop. This loop is oriented by the orientation of the plane. Let $a$ (resp. $b$ ) be the sign which is equal to + if and only if the orientation of the top branch (resp. the bottom branch) agrees with the orientation of $\Gamma$. Let $h$ be the commun $D^{\prime}$-sign of the created crossings. We say that $f$ is a Reidemeister move of type $\mathrm{II}^{+}(a, b, h)$ and that $g$ is a Reidemeister move of type $\mathrm{II}^{-}(a, b, h)$. In this case the diagram $D^{\prime}$ is oriented as follows:

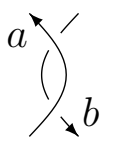

For technical reasons we'll say also that $f$ is a Reidemeister move of type $\operatorname{II}^{e}\left(e^{\prime}, a, b, h\right)$ if $f$ is a Reidemeister move of type $\mathrm{II}^{e}(a, b, h)$ (resp. $\left.\mathrm{II}^{e}(b, a, h)\right)$ if $e^{\prime}=+$ (resp. $\left.e^{\prime}=-\right)$.

Let $f: D \rightarrow D^{\prime}$ be a Reidemeister move of type $\mathrm{III}_{e}$ between oriented diagrams. Let $\Theta$ be the triangle in $D$ modified by $f$. This triangle is oriented by the orientation of the plane. Let $a$ (resp. $b, c$ ) be the sign which is equal to + if and only if the orientation of the top branch (resp. the middle branch, the bottom branch) agrees with the orientation of the triangle. Let $h$ be the $D$-sign of the center of $\Theta$. We say that $f$ is a Reidemeister move of type $\operatorname{III}(e, a, b, c, h)$. The diagram $D$ is oriented as follows:

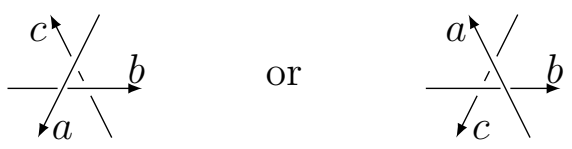

depending if $e=+$ or $e=-$.

It is easy to see that the inverse move of $f$ is a Reidemeister move of type $\operatorname{III}(e,-a,-b,-c,-h)$. 
Let $f: D \rightarrow D^{\prime}$ be a surgery move of index 0 between oriented diagrams and $g$ be its inverse move. Let $a$ be the winding number of the created circle and $h$ be the $D$-sign of a point in this circle. We say that $f$ is a surgery move of type $(0, a, h)$ and that $g$ is a surgery move of type $(2, a, h)$.

Let $f: D \rightarrow D^{\prime}$ be a surgery move of index 1 between oriented diagrams. Let $\gamma$ be a path inducing this surgery. Let $a$ be the sign which is equal to + if and only if $\gamma$ is on the left of $D$ and $h$ be the $D$-sign of the middle point in $\gamma$. We say that $f$ is a surgery move of type $(1, a, h)$. It is easy to see that the inverse move of $f$ is a surgery move of type $(1,-a,-h)$.

\subsection{The correspondance $f \mapsto f^{1}$.}

Consider an elementary move $f: D \rightarrow D^{\prime}$ where $D$ and $D^{\prime}$ are oriented link diagrams. This move is called an elementary move of oriented diagrams if $D$ and $D^{\prime}$ have the same orientation outside the modification area.

Here we'll associate to each elementary move $f: D \rightarrow D^{\prime}$ of oriented diagrams a morphism $f^{1}$ from $K H(D)$ to $K H\left(D^{\prime}\right)$.

Denote by $X_{-}$and $X_{-}^{\prime}$ the set of negative crossings of $D$ and $D^{\prime}$.

Suppose the move is a Reidemeister move of type $\mathrm{I}_{-}^{+}$or $\mathrm{II}^{+}$. In this case $X_{-}^{\prime}$ is the union of $X_{-}$and one crossing $x$ and the map $f^{1}$ is defined by:

$$
\forall(u, v) \in k h(D) \times \Lambda^{+}\left(X_{-}\right), \quad f^{1}(u \otimes v)=(-1)^{|u|} f^{0}(u) \otimes x \wedge v
$$

where $|u|$ is the degree of $u$.

Suppose the move is a Reidemeister move of type $\mathrm{I}_{-}^{-}$or $\mathrm{II}^{-}$. In this case $X_{-}$is the union of $X_{-}^{\prime}$ and one crossing $x$ and the map $f^{1}$ is defined by:

$$
\forall(u, v) \in k h(D) \times \Lambda^{+}\left(X_{-}^{\prime}\right), \quad f^{1}(u \otimes x \wedge v)=-(-1)^{|u|} f^{0}(u) \otimes v
$$

In all other cases, $X_{-}$and $X_{-}^{\prime}$ are the same and the map $f^{1}$ is defined by:

$$
\forall(u, v) \in k h(D) \times \Lambda^{+}\left(X_{-}\right), \quad f^{1}(u \otimes v)=f^{0}(u) \otimes v
$$

It is not difficult to see that each map $f^{1}$ is a morphism of complexes of degree 0 . Moreover is $f$ is a Reidemeister move with inverse move $g, g^{1}$ is a homotopy inverse of $f^{1}$.

Notice that each map $f^{0}$ and each map $f^{1}$ are invariant under any endomorphism of $R$.

\subsection{The correspondance $f \mapsto f^{\mathscr{K}}$.}

Consider elements $A(e, a, h), X(a, h), Y(a, h), Z(a, h)$ in $R^{*}$, elements $B(a, b, h)$ in $(R \otimes R)^{*}$ and elements $C(e, a, b, c)$ in $(R \otimes R \otimes R)^{*}$, depending on signs $e, a, b, c, h$. Such a data $\mathscr{K}=(A, B, C, X, Y, Z)$ will be called a Khovanov data. Using this data we'll construct a correspondance associating to each elementary move $f: D \rightarrow D^{\prime}$ a morphism $f^{\mathscr{K}}: K H(D) \rightarrow K H\left(D^{\prime}\right)$. 
Let $f: D \rightarrow D^{\prime}$ be a Reidemeister move of type $\mathrm{I}^{+}(e, a, h)$ and $g$ be its inverse move. Let $p \in D$ be a point in the modified branch of $D$. The morphisms $f^{\mathscr{K}}$ and $g^{\mathscr{K}}$ are defined by:

$$
f^{\mathscr{K}}=f^{1} \circ \widehat{T}_{p}(A(e, a, h)) \quad g^{\mathscr{K}}=\widehat{T}_{p}\left(A(e, a, h)^{-1}\right) \circ g^{1}
$$

Let $f: D \rightarrow D^{\prime}$ be a Reidemeister move of type $\mathrm{II}^{+}(a, b, h)$ and $g$ be its inverse move. Let $p$ be a point in the top branch and $q$ be a point in the bottom branch. The morphisms $f^{\mathscr{K}}$ and $g^{\mathscr{K}}$ are defined by:

$$
f^{\mathscr{K}}=f^{1} \circ \widehat{T}(B(a, b, h)) \quad g^{\mathscr{K}}=\widehat{T}\left(B(a, b, h)^{-1}\right) \circ g^{1}
$$

where $\widehat{T}$ is the map: $u \otimes v \mapsto \widehat{T}_{p}(u) \widehat{T}_{q}(v)$.

Let $f: D \rightarrow D^{\prime}$ be a Reidemeister move of type $\operatorname{III}(e, a, b, c, h)$ and $g$ be its inverse move. The move $g$ is a Reidemeister move of type $\operatorname{III}(e,-a,-b,-c,-h)$. Let $B_{1}$ (resp. $\left.B_{2}, B_{3}\right)$ be the top branch (resp. the middle branch, the bottom branch) near the modified triangle in $D$. For $i=1,2,3$, denote by $p_{i}$ a point in $B_{i}$ and denote by $\widehat{T}$ the map: $u \otimes v \otimes w \mapsto \widehat{T}_{p_{1}}(u) \widehat{T}_{p_{2}}(v) \widehat{T}_{p_{3}}(w)$. Suppose that $D(e, a, b, c, h)$ are elements in $(R \otimes R \otimes R)^{*}$ then we can set: $\widehat{f}=f^{1} \circ \widehat{T}(D(e, a, b, c, h))$. But the condition: $\widehat{f} \circ \widehat{g} \sim$ Id is equivalent to the condition:

$$
D(e, a, b, c, h) D(e,-a,-b,-c,-h)=1
$$

and that's equivalent to the fact that $D(e, a, b, c, h)$ is on the form $D^{\prime}(e, a h, b h, c h)^{h}$. So we define $f^{\mathscr{K}}$ by:

$$
f^{\mathscr{K}}=f^{1} \circ \widehat{T}\left(C(e, a h, b h, c h)^{h}\right)
$$

Let $f: D \rightarrow D^{\prime}$ be a surgery move of type $(0, a, h)$ and $g$ be its inverse move. Let $p$ be a point in the created circle. Then we define $f^{\mathscr{K}}$ and $g^{\mathscr{K}}$ by:

$$
f^{\mathscr{K}}=\widehat{T}_{p}(X(a, h)) \circ f^{1} \quad g^{\mathscr{K}}=g^{1} \circ \widehat{T}_{p}(Z(a, h))
$$

Let $f: D \rightarrow D^{\prime}$ be a surgery move of type $(1, a, h)$. Let $p$ be a point in the boundary of the surgery path. Then $f^{\mathscr{K}}$ is defined by:

$$
f^{\mathscr{K}}=f^{1} \circ \widehat{T}_{p}(Y(a, h))
$$

It is easy to see that, for every elementary move $f, f^{\mathscr{K}}$ is a morphism of degree 0 well defined up to homotopy. Moreover, if $g$ is the inverse move of a Reidemeister move $f, g^{\mathscr{K}}$ is a homotopy inverse of $f^{\mathscr{K}}$.

\section{Movie moves.}

In all this section $\mathscr{K}=(A, B, C, X, Y, Z)$ is a given Khovanov data. For technical reasons we define the elements $B^{e}(a, b, h)$ by:

$$
B(a, b, h)=u \otimes v \quad \Longrightarrow \quad B^{0}(a, b, h)=u v \quad B^{+}(a, b, h)=u \otimes v \quad B^{-}(b, a, h)=v \otimes u
$$


Let $\mathscr{D}$ be the set of link diagrams or the set of oriented link diagrams and $A$ be a correspondance associating to each $D \in \mathscr{D}$ a $K$-complex $A(D)$.

In $\mathscr{D}$ we have elementary moves and every elementary move $f: D \rightarrow D^{\prime}$ has an inverse move: $\bar{f}: D^{\prime} \rightarrow D$.

Consider diagrams $D_{0}, D_{1}, \ldots, D_{n}$ in $\mathscr{D}$ and elementary moves $f_{i}: D_{i-1} \rightarrow D_{i}$. Such a sequence $\varphi=\left(D_{0}, D_{1}, \ldots D_{n}\right)$ (or $\left.\varphi=\left(f_{1}, f_{2}, \ldots f_{n}\right)\right)$ will be called a movie sequence from $D_{0}$ to $D_{n}$. If $D=D_{0}$ and $D^{\prime}=D_{n}$ are the diagrams of two links $L$ and $L^{\prime}$, the movie sequence $\varphi$ induces a cobordism from $L$ to $L^{\prime}$ and this cobordism is oriented in the oriented case. By replacing every elementary moves by its inverse, we get a new movie sequence: $\bar{\varphi}=\left(\bar{f}_{n}, \ldots, \bar{f}_{1}\right)$ from $D^{\prime}$ to $D$ and the cobordism associated to $\bar{\varphi}$ is isotopic to the opposite of the cobordism associated to $\varphi$.

Suppose all the elementary moves of a movie sequence $\varphi$ are Reidemeister moves. Then the movie sequence induces an isotopy from $L$ to $L^{\prime}$. Suppose also $D$ is equal to $D^{\prime}$ and the isotopy from $L$ to $L^{\prime}=L$ is isotopic to the identity. In this case the movie sequence $\varphi$ will be called a movie move of type I.

Consider $\varphi=\left(D_{0}, D_{1}, \ldots D_{p}\right)$ and $\psi=\left(D_{0}^{\prime}, D_{1}^{\prime}, \ldots, D_{q}^{\prime}\right)$ two movie sequences from $D_{0}=D_{0}^{\prime}$ to $D_{p}=D_{q}^{\prime}$. The diagrams $D_{0}$ and $D_{p}$ are the diagrams of two links $L$ and $L^{\prime}$ and the two movie sequences induce two cobordisms from $L$ to $L^{\prime}$. Suppose these two cobordisms are isotopic. Then the pair $(\varphi, \psi)$ will be called a movie move of type II.

Consider a correspondance $f \mapsto \widehat{f}$ associating to every elementary move $f: D \rightarrow$ $D^{\prime}$ in $\mathscr{D}$ a morphism $\widehat{f}$ from $A(D)$ to $A\left(D^{\prime}\right)$ and suppose that this correspondance has the following property: for every Reidemeister move $f$ with inverse move $g, \widehat{g}$ is a homotopy inverse of $\widehat{f}$.

Let $\varphi=\left(f_{1}, f_{2}, \ldots, f_{n}\right)$ be a movie sequence from $D$ to $D^{\prime}$. Then we have a map $\widehat{\varphi}=\widehat{f}_{n} \circ \ldots \circ \widehat{f}_{1}$ from $A(D)$ to $A\left(D^{\prime}\right)$.

Suppose this correspondance is coming from a functor from the category of cobordisms of links to the homotopy category of $K$-complexes. In this case, for each movie move $\varphi$ of type I, the morphism $\widehat{\varphi}$ is homotopic to the identity and for each movie move $(\varphi, \psi)$ of type II, the two morphisms $\widehat{\varphi}$ and $\widehat{\psi}$ are homotopic.

Let $\varphi$ be a movie move of type I. Then the morphism $\widehat{\bar{\varphi}}$ is a homotopy inverse of $\widehat{\varphi}$ and the condition " $\widehat{\varphi}$ is homotopic to the identity" implies the same condition for the morphism $\widehat{\bar{\varphi}}$.

But if $(\varphi, \psi)$ is a movie move of type II, we get two conditions: $\widehat{\varphi}$ and $\widehat{\psi}$ are homotopic and $\widehat{\bar{\varphi}}$ and $\widehat{\bar{\psi}}$ are also homotopic.

So a movie move of type I induces one condition on the correspondance and a movie move of type II induces two conditions.

We'll apply this construction in three cases:

- $\mathscr{D}$ is the set of link diagrams, $A(D)$ is the Khovanov complex $k h(D)$ and the correspondance is: $f \mapsto f^{0}$.

- $\mathscr{D}$ is the set of oriented link diagrams, $A(D)$ is the Khovanov complex $K H(D)$ and the correspondance is: $f \mapsto f^{1}$.

- $\mathscr{D}$ is the set of oriented link diagrams, $A(D)$ is the Khovanov complex $K H(D)$ and the correspondance is: $f \mapsto f^{\mathscr{K}}$. 
4.0 Notation: From now on we'll use the following notation: for every element $u \in R$ and every sign $e$ we set:

$$
u^{(e)}= \begin{cases}u & \text { if } e=+ \\ \bar{u} & \text { if } e=-\end{cases}
$$

4.1 Movie moves of type $\mathbf{M V M}_{0}$. Consider a link diagram $D$ and two elementary moves $f$ and $g$ modifying $D$ on disjoint areas. Let $D_{1}$ be the diagram $D$ modified by $f$ and $D_{2}$ be the diagram $D$ modified by $g$. The move $f$ (resp. $g$ ) induces a well defined move $f^{\prime}$ (resp. $g^{\prime}$ ) on $D_{2}$ (resp. $D_{1}$ ) and we have a commutative diagram:

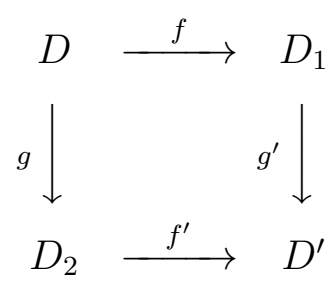

So we get two movie sequences: $\varphi=\left(f, g^{\prime}\right)$ and $\psi=\left(g, f^{\prime}\right)$ and a movie move $(\varphi, \psi)$ of type II. This movie move will be called a movie move of type $\mathrm{MVM}_{0}$.

4.1.a Lemma: Let $(\varphi, \psi)$ be a movie move of type $M V M_{0}$ associated to elementary moves $f$ and $g$. Then we have:

$$
\varphi^{0}=(-1)^{p q} \psi^{0}
$$

where $p$ and $q$ are the degrees of the map $f^{0}$ and $g^{0}$.

In the oriented case we have:

$$
\varphi^{1}=\psi^{1} \quad \varphi^{\mathscr{K}}=\psi^{\mathscr{K}} \quad \bar{\varphi}^{\mathscr{K}}=\bar{\psi}^{\mathscr{K}}
$$

Proof: It is easy to see that we have some commutativity in the graded sense and that implies the first formula and therefore the other ones.

For example, consider the case where $f$ and $g$ are Reidemeister moves of type $\mathrm{I}^{+}(-, a, h)$ and $\mathrm{I}^{+}(-, b, k)$. Let $x$ (resp. $\left.y\right)$ be the crossing created by $f$ (resp. $g$ ) and $p$ (resp. $q$ ) be a regular point in $D$ near $x$ (resp. near $y$ ). Let $X_{-}$be the set of negative crossings of the diagram $D$. So, for every $u \in k h(D)$ and every $v \in \Lambda^{+}\left(X_{-}\right)$, we have:

$$
\begin{gathered}
\varphi^{0}(u)=f^{0}(y \otimes u \otimes 1)=x \wedge y \otimes u \otimes 1 \otimes 1 \\
\psi^{0}(u)=g^{0}(x \otimes u \otimes 1)=y \wedge x \otimes u \otimes 1 \otimes 1 \Longrightarrow \psi^{0}=-\varphi^{0} \\
\varphi^{1}(u \otimes v)=f^{1}\left(g^{\prime 0}(u) \otimes y \wedge v\right)=\varphi^{0}(u) \otimes x \wedge y \wedge v \\
\psi^{1}(u \otimes v)=g^{1}\left(f^{\prime 0}(u) \otimes x \wedge v\right)=\psi^{0}(u) \otimes y \wedge x \wedge v \Longrightarrow \psi^{1}=\varphi^{1}
\end{gathered}
$$




$$
\varphi^{\mathscr{K}}=\varphi^{1} \circ \widehat{T}_{p}(A(-, a, h)) \widehat{T}_{q}(A(-, b, k))=\psi^{1} \circ \widehat{T}_{q}(A(-, b, k)) \widehat{T}_{p}(A(-, a, h))=\psi^{\mathscr{K}}
$$

The last formula $\bar{\varphi}^{\mathscr{K}}=\bar{\psi}^{\mathscr{K}}$ is just the formula $\varphi^{\mathscr{K}}=\psi^{\mathscr{K}}$ applied for Reidemeister moves $\bar{g}$ and $\bar{f}$.

4.2 Movie moves of type $\mathbf{M V M}_{1}$. Consider a link diagram $D$ and a regular point $p$ in $D$. Let $e$ be a sign. We may apply a Reidemeister move of type $\mathrm{I}_{e}^{+}$on $D$ near $p$, then a new Reidemeister move of type $\mathrm{I}_{-e}^{+}$in the created loop. By applying a Reidemeister move of type $\mathrm{II}^{-}$we may remove the two created crossings and recover the diagram $D$. So we have a movie move of type I. This move will be called a movie move of type $\mathrm{MVM}_{1}(e)$ near $p$.

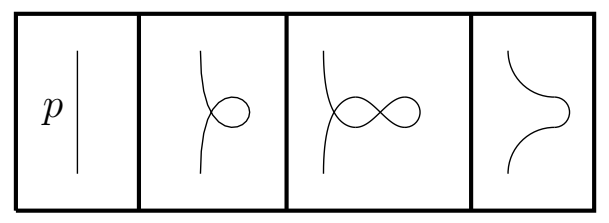

4.2.a Lemma: Let $\varphi$ be a movie move of type $\operatorname{MVM}_{1}(e)$ near a point $p \in D$. Then we have:

$$
\varphi^{0}=-e T_{p}\left(\frac{\omega}{\omega^{(e)}}\right)
$$

In the oriented case, suppose the first move is a Reidemeister move of type $I^{+}(e, a, h)$. Then we have:

$$
\begin{gathered}
\varphi^{1}=-e T_{p}\left(\frac{\omega}{\omega^{(e)}}\right)=-e \widehat{T}_{p}\left(\frac{\omega^{(a h)}}{\omega^{(e a h)}}\right) \\
\varphi^{\mathscr{K}}=-e \widehat{T}_{p}\left(\frac{\omega^{(a h)}}{\omega^{(e a h)}} \frac{A(e, a, h) A(-e,-a, h)}{B^{0}(a, a, h)}\right)
\end{gathered}
$$

Proof: A straightforward computation gives the following:

$$
\begin{gathered}
e=+\quad \Longrightarrow \quad \varphi^{0}=-\mathrm{Id} \\
e=-\Longrightarrow \varphi^{0}=T_{p}\left(\frac{\omega}{\bar{\omega}}\right)
\end{gathered}
$$

So we get the first formula. The second formula: $\varphi^{1}=-e T_{p}\left(\omega / \omega^{(e)}\right)$ is easy to deduce.

Since the first move is a Reidemeister move of type $\mathrm{I}^{+}(e, a, h)$, the orientation of $D$ is given by the following:

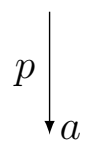

So the second move is a Reidemeister move of type $\mathrm{I}^{+}(-e,-a, h)$ and the last one is a Reidemeister move of type $\mathrm{II}^{-}(a, a, h)$. The result follows. 
4.3 Movie moves of type $\mathbf{M V M}_{2}$. Let $D$ be a link diagram and $x$ be a crossing of $D$. We may modify $D$ by a Reidemeister move of type $\mathrm{II}^{+}$which creates two new crossings $y$ and $z$ and then remove $x$ and $y$ by a Reidemeister move of type $\mathrm{II}^{-}$.

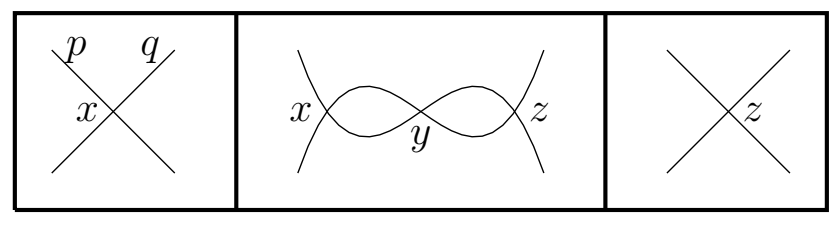

So we get a movie move. We say that this move is a movie move of type $\operatorname{MVM}_{2}(e)$ near $(p, q)$ where $e=+$ (resp. $e=-)$ if the branch containing $p$ is over (resp. under) the branch containing $q$.

4.3.a Lemma: Let e be a sign and $\varphi$ be a movie move of type $M_{V M}(e)$ near $(p, q)$. Then we have:

$$
\varphi^{0}=e T_{p}(\omega) T_{q}\left(\omega^{-1}\right)
$$

In the oriented case, suppose the first move is a Reidemeister move of type $I I^{+}(e, a, b, h)$. Then we have:

$$
\begin{gathered}
\varphi^{1}=-a b T_{p}(\omega) T_{q}\left(\omega^{-1}\right)=-a b \widehat{T}\left(\omega^{(-b h)} \otimes \frac{1}{\omega^{(a h)}}\right) \\
\varphi^{\mathscr{K}}=-a b \widehat{T}\left(\left(\omega^{(-b h)} \otimes \frac{1}{\omega^{(a h)}}\right) \frac{B^{e}(a, b, h)}{B^{e}(-a,-b, h)}\right)
\end{gathered}
$$

where $\widehat{T}$ is the map $u \otimes v \mapsto \widehat{T}_{p}(u) \widehat{T}_{q}(v)$.

Proof: A straightforward computation gives the first formula. Let $f$ and $g$ be the Reidemeister moves in $\varphi$ and $X_{-}$be the set of negative crossings of $D$.

So it is easy to see that the orientation of the second diagram is given by:

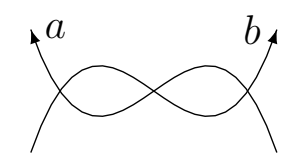

and the two moves are Reidemeister moves of type $\mathrm{II}^{+}(e, a, b, h)$ and $\mathrm{II}^{-}(e,-a,-b, h)$. Moreover the signs of the crossings $x, y, z$ are: $-e a b, e a b,-e a b$.

If $e a b=+, x$ is in $X_{-}$and we have, for every $u \in k h(D)$ and every $x \wedge v \in \Lambda^{+}\left(X_{-}\right)$:

$$
\begin{aligned}
\varphi^{1}(u \otimes x \wedge v) & =(-1)^{|u|} g^{1}\left(f^{0}(u) \otimes z \wedge x \wedge v\right)=-(-1)^{|u|} g^{1}\left(f^{0}(u) \otimes x \wedge z \wedge v\right) \\
= & (-1)^{\left|f^{0}(u)\right|}(-1)^{|u|} g^{0}(u) \otimes z \wedge v=-\varphi^{0}(u) \otimes z \wedge v
\end{aligned}
$$

and that implies:

$$
\varphi^{1}=-a b T_{p}(\omega) T_{q}\left(\omega^{-1}\right)
$$

If $e a b=-, x$ is not in $X_{-}$and we have, for every $u \in k h(D)$ and every $v \in$ $\Lambda^{+}\left(X_{-}\right)$:

$$
\varphi^{1}(u \otimes v)=(-1)^{|u|} g^{1}\left(f^{0}(u) \otimes y \wedge v\right)=-(-1)^{\left|f^{0}(u)\right|}(-1)^{|u|} g^{0}(u) \otimes v=\varphi^{0}(u) \otimes v
$$


and that implies again:

$$
\varphi^{1}=-a b T_{p}(\omega) T_{q}\left(\omega^{-1}\right)
$$

On the other hand, the $D$-sign of a point in the plane between $p$ and $q$ is $a b h$. So we get:

$$
\varphi^{1}=-a b \widehat{T}_{p}\left(\omega^{(-b h)}\right) \widehat{T}_{q}\left(\frac{1}{\omega^{(a h)}}\right)=-a b \widehat{T}\left(\omega^{(-b h)} \otimes \frac{1}{\omega^{(a h)}}\right)
$$

and the last formula follows easily.

4.4 Movie moves of type $\mathrm{MVM}_{3}$. Let $D$ be a link diagram and $p$ and $q$ be two regular points in $D$. Suppose that these two points may be joined by a path outside of $D$. Then we have a movie move described by the following figure:

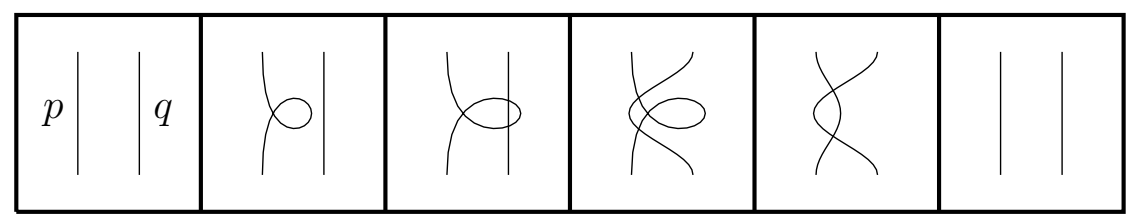

This movie move depends on two sign $e$ and $e^{\prime}$. The sign $e$ is the sign of the first Reidemeister move of type I and $e^{\prime}$ is + if and only if the loop goes over the branch containing $q$. We say that this move is a movie move of type $\operatorname{MVM}_{3}\left(e, e^{\prime}\right)$ near $(p, q)$.

4.4.a Lemma: Let $e$ and $e^{\prime}$ be two signs. Let $p$ and $q$ be two points in a link diagram $D$ and $\varphi$ be a movie move of type $M_{V M}\left(e, e^{\prime}\right)$ near $(p, q)$. Then we have:

$$
\varphi^{0}=T_{p}\left(\frac{\omega}{\omega^{(e)}}\right)
$$

In the oriented case, let $a, b, h$ be the signs such that the first move of $\varphi$ is a Reidemeister move of type $I^{+}(e, a, h)$ and $a$ is equal to $b$ if and only if the two branches are oriented in the same way. Then we have:

$$
\begin{gathered}
\varphi^{1}=e T_{p}\left(\frac{\omega}{\omega^{(e)}}\right)=e \widehat{T}_{p}\left(\frac{\omega^{(a h)}}{\omega^{(e a h)}}\right) \\
\varphi^{\mathscr{K}}=e \widehat{T}\left(\left(\frac{\omega^{(a h)}}{\omega^{(e a h)}} \otimes 1\right)\left(\frac{A(e, a, h)}{A(e, a,-h)} \otimes 1\right) \frac{B^{e^{\prime}}(a, b,-a b h)}{B^{e^{\prime}}(-a, b, a b h)} C\right)
\end{gathered}
$$

where $\widehat{T}$ is the map $u \otimes v \mapsto \widehat{T}_{p}(u) \widehat{T}_{q}(v)$ and $C$ is defined by:

$$
\begin{aligned}
& e^{\prime}=+, \quad C(e,-a h,-a h, b h)=u \otimes v \otimes w \quad \Longrightarrow \quad C=(u v)^{-h} \otimes w^{-h} \\
& e^{\prime}=-, \quad C(e, b h,-a h,-a h)=u \otimes v \otimes w, \quad \Longrightarrow \quad C=(w v)^{-h} \otimes u^{-h}
\end{aligned}
$$

Proof: A straightforward computation gives the first formula.

The diagram $D$ is oriented as follows:

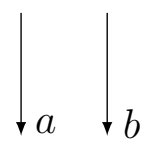


Denote by $\left(f_{1}, f_{2}, f_{3}, f_{4}, f_{5}\right)$ the sequence $\varphi$. Then the types of $f_{1}, f_{2}, f_{3}, f_{4}, f_{5}$ are:

$$
\mathrm{I}^{+}(e, a, h), \mathrm{II}^{+}(a, b,-a b h), \operatorname{III}(e, a, a,-b,-h), \mathrm{I}^{-}(e, a,-h), \mathrm{II}^{-}(-a, b, a b h)
$$

if $e^{\prime}=+$ and:

$$
\mathrm{I}^{+}(e, a, h), \mathrm{II}^{+}(b, a,-a b h), \operatorname{III}(e,-b, a, a,-h), \mathrm{I}^{-}(e, a,-h), \mathrm{II}^{-}(b,-a, a b h)
$$

if $e^{\prime}=-$.

In the case: $e=+$, only $f_{2}$ and $f_{5}$ modify the set of negative crossings. Then, during the move $\varphi$, only one negative crossing appears and then disappears. So we get:

$$
\varphi^{1}=T_{p}\left(\frac{\omega}{\omega^{(e)}}\right)
$$

In the case: $e=-, f_{1}$ creates a negative crossing $x, f_{2}$ creates a negative crossing $y$, $f_{4}$ removes $x$ and $f_{5}$ removes $y$. This operation produces a sign and we have:

$$
\varphi^{1}=-T_{p}\left(\frac{\omega}{\omega^{(e)}}\right)
$$

So we get the second formula. The other formulae are easy to check.

4.5 Movie moves of type $\mathrm{MVM}_{4}$. Consider a link diagrams and two consecutive crossings $x$ and $y$. Then we have a movie move described by the following figure:

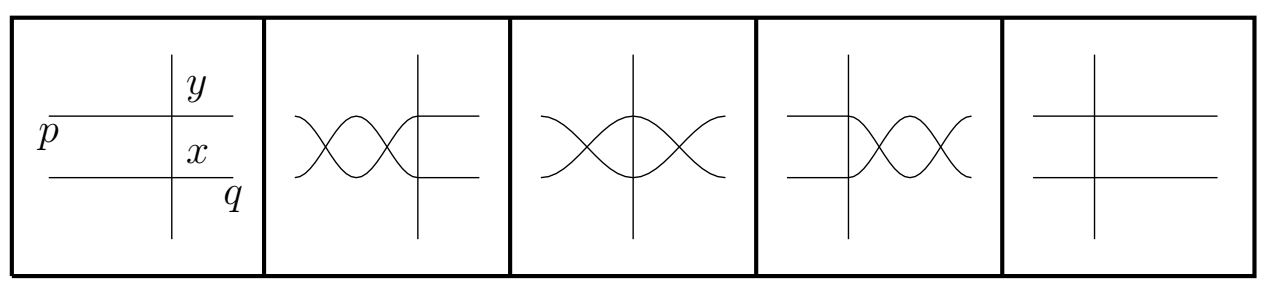

Up to replacing this movie move by its inverse we may as well suppose that the horizontal branch containing $y$ goes over the other horizontal branch after the first Reidemeister move. So this move depends only on an element $e \in\{0,+,-\}$ where $e=+$ (resp. $e=0, e=-$ ) if the vertical branch is over (resp. between, under) the two other branches. We say that this move is a movie move of type $\mathrm{MVM}_{4}(e)$ near $(p, q)$.

4.5.a Lemma: Let $p$ and $q$ be two points in a link diagram $D$. Let $\varphi$ be a movie move of type $M_{V M}(e)$ near $(p, q)$. Then the morphism $\varphi^{0}$ is homotopic to the operator:

$$
\left(1-2 e^{2}\right) T_{p}\left(\omega^{-1}\right) T_{q}(\omega)
$$

In the oriented case, suppose that the first move in $\varphi$ is a Reidemeister move of type $\mathrm{II}^{+}(a, b, h)$ and that $c=+($ resp. $c=-)$ if the vertical branch is oriented downward (resp. upward). Then we have:

$$
\varphi^{1} \sim\left(1-2 e^{2}\right) T_{p}\left(\omega^{-1}\right) T_{q}(\omega)=\left(1-2 e^{2}\right) \widehat{T}_{p}\left(\frac{1}{\omega^{(b h)}}\right) \widehat{T}_{q}\left(\omega^{(-a h)}\right)
$$




$$
\varphi^{\mathscr{K}} \sim\left(1-2 e^{2}\right) \widehat{T}\left(\left(\frac{1}{\omega^{(b h)}} \otimes \omega^{(-a h)} \otimes 1\right)\left(\frac{B(a, b, h)}{B(a, b,-h)} \otimes 1\right) C^{-a b h}\right)
$$

where $\widehat{T}$ is the map $u \otimes v \otimes w \mapsto \widehat{T}_{p}(u) \widehat{T}_{q}(v) \widehat{T}_{r}(w)$ (for any $r$ in the vertical branch) and $C$ is defined by:

$e=-, \quad C(+, b h, a h, a b c h) C(-,-b h,-a h, a b c h)=u \otimes v \otimes w \quad \Longrightarrow \quad C=u \otimes v \otimes w$

$e=0, \quad C(-, b h, a b c h, a h) C(+,-b h, a b c h,-a h)=u \otimes v \otimes w \quad \Longrightarrow \quad C=u \otimes w \otimes v$

$e=+, C(+, a b c h, b h, a h) C(-, a b c h,-b h,-a h)=u \otimes v \otimes w \quad \Longrightarrow \quad C=w \otimes u \otimes v$

Proof: The union of the branches is a graph with six points in its boundary. Take a counterclockwise numbering of these points begining with the point $p$. So we have: $p_{1}=p, p_{4}=q$. The operator $T_{p_{i}}$ will be denoted by $T_{i}$.

There are four complexes $U, V, W$ and $H$ such that:

$$
k h(D)=1 \otimes U \oplus x \otimes V \oplus y \otimes W \oplus x \wedge y \otimes H
$$

Using small paths near $x$ or $y$, we get surgery maps $\gamma_{x}$ and $\gamma_{y}$. These maps are morphisms of complexes. The morphism $\gamma_{x}$ is a map from $U$ to $V$, from $V$ to $U$, from $W$ to $H$ and from $H$ to $W$. The morphism $\gamma_{y}$ is a map from $U$ to $W$, from $W$ to $U$, from $V$ to $H$ and from $H$ to $V$. A straightforward computation shows the following:

If $\varphi$ is a movie move of type $\operatorname{MVM}_{4}(-)$, we have (for $u \in U, v \in V, w \in W$, $h \in H)$ :

$$
\begin{gathered}
\varphi^{0}(1 \otimes u)=-1 \otimes u \\
\varphi^{0}(x \otimes v)=-x \otimes T_{1}(\bar{\omega}) T_{3}\left(\omega^{-1}\right)(v)+\varepsilon\left(\omega^{2}\right) y \otimes T_{1}\left(\omega^{-2}\right) \gamma_{x} \gamma_{y}(v) \\
\varphi^{0}(y \otimes w)=y \otimes\left(-1+T_{1}\left(\omega^{-1}\right) T_{3}(\bar{\omega})-T_{1}\left(\omega^{-1}\right) T_{3}(\omega)\right)(w)-\varepsilon\left(\omega^{2}\right) x \otimes T_{1}\left(\omega^{-2}\right) \gamma_{x} \gamma_{y}(w) \\
\varphi^{0}(x \wedge y \otimes h)=-x \wedge y \otimes h
\end{gathered}
$$

If $\varphi$ is a movie move of type $\operatorname{MVM}_{4}(0)$, we have:

$$
\begin{gathered}
\varphi^{0}(1 \otimes u)=1 \otimes T_{3}(\omega) T_{5}\left(\bar{\omega}^{-1}\right)(u) \\
\varphi^{0}(x \otimes v)=x \otimes T_{1}(\omega) T_{5}\left(\bar{\omega}^{-1}\right)(v)-\varepsilon(1) y \otimes \gamma_{x} \gamma_{y}(v) \\
\varphi^{0}(y \otimes w)=y \otimes w \\
\varphi^{0}(x \wedge y \otimes h)=x \wedge y \otimes T_{3}\left(\omega^{-1}\right) T_{5}(\omega)(h)
\end{gathered}
$$

If $\varphi$ is a movie move of type $\mathrm{MVM}_{4}(+)$, we have:

$$
\begin{gathered}
\varphi^{0}(1 \otimes u)=-1 \otimes T_{1}(\bar{\omega}) T_{3}\left(\omega^{-1}\right)(u) \\
\varphi^{0}(x \otimes v)=-x \otimes T_{1}\left(\omega^{-1}\right) T_{5}(\omega)(v) \\
\varphi^{0}(y \otimes w)=-y \otimes T_{1}(\bar{\omega}) T_{3}\left(\omega^{-1}\right)(w)+\varepsilon\left(\omega^{2}\right) x \otimes T_{1}\left(\omega^{-2}\right) \gamma_{x} \gamma_{y}(w) \\
\varphi^{0}(x \wedge y \otimes h)=-x \wedge y \otimes h
\end{gathered}
$$


Consider the maps $k_{x}$ and $k_{y}$ from $k h(D)$ to $k h(D)$ that vanish on $1 \otimes U \oplus x \otimes$ $V \oplus y \oplus W$ and satisfy the following:

$$
k_{x}(x \wedge y \otimes h)=x \otimes \gamma_{y}(h) \quad k_{y}(x \wedge y \otimes h)=y \otimes \gamma_{x}(h)
$$

for every $h \in H$. These maps induce the following homotopies:

$$
h_{x}=d\left(k_{x}\right)=d \circ k_{x}+k_{x} \circ d \quad h_{y}=d\left(h_{y}\right)=d \circ k_{y}+k_{y} \circ d
$$

and we have:

$$
\begin{aligned}
h_{x}(1 \otimes u)=0 & h_{y}(1 \otimes u)=0 \\
h_{x}(x \otimes v)=-x \otimes \gamma_{y}^{2}(v) & h_{y}(x \otimes v)=-y \otimes \gamma_{x} \gamma_{y}(v) \\
h_{x}(y \otimes w)=x \otimes \gamma_{x} \gamma_{y}(w) & h_{y}(y \otimes w)=y \otimes \gamma_{x}^{2}(w) \\
h_{x}(x \wedge y \otimes h)=-x \wedge y \otimes \gamma_{y}^{2}(h) & h_{y}(x \wedge y \otimes h)=x \wedge y \otimes \gamma_{x}^{2}(h)
\end{aligned}
$$

Moreover one checks that $\gamma_{x}^{2}$ (resp. $\left.\gamma_{y}^{2}\right)$ is on $W$ and $H$ (resp. on $V$ and $H$ ) the map $T_{2}(\omega) T_{4}(\alpha)-T_{2}(\omega \bar{\alpha})$ (resp. the map $T_{1}(\omega) T_{5}(\alpha)-T_{1}(\omega \bar{\alpha})$ ). So we get:

$$
\varphi^{0}=\varepsilon(1) T_{1}(\bar{\omega}) T_{3}\left(\omega^{-1}\right) h_{x}-\varepsilon\left(\omega^{2}\right) T_{1}\left(\omega^{-2}\right) h_{y}-T_{2}\left(\omega^{-1}\right) T_{5}(\omega)
$$

in the first case,

$$
\varphi^{0}=\varepsilon(1) h_{y}+T_{4}(\omega) T_{5}\left(\bar{\omega}^{-1}\right)
$$

in the second case and:

$$
\varphi^{0}=\varepsilon\left(\omega^{2}\right) T_{1}\left(\omega^{-2}\right) h_{x}-T_{1}(\bar{\omega}) T_{2}\left(\omega^{-1}\right)
$$

in the last case. If we denote by $\sim$ the homotopy relation, we get:

$$
\varphi^{0} \sim-T_{2}\left(\omega^{-1}\right) T_{5}(\omega) \sim-T_{4}\left(\bar{\omega}^{-1}\right) T_{1}(\bar{\omega})=-T_{1}\left(\omega^{-1}\right) T_{4}(\omega)
$$

in the first case,

$$
\varphi^{0} \sim T_{4}(\omega) T_{5}\left(\bar{\omega}^{-1}\right) \sim T_{1}\left(\omega^{-1}\right) T_{4}(\omega)
$$

in the second case and:

$$
\varphi^{0} \sim-T_{1}(\bar{\omega}) T_{2}\left(\omega^{-1}\right) \sim-T_{1}(\bar{\omega}) T_{4}\left(\bar{\omega}^{-1}\right)=-T_{1}\left(\omega^{-1}\right) T_{4}(\omega)
$$

in the last case. So we get the first formula.

The movie move creates one negative crossing and then remove it. Therefore we get the same formula for $\varphi^{1}$.

The second diagram $D^{\prime}$ is oriented as follows:

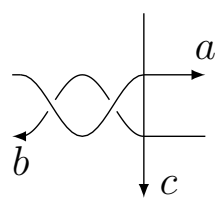


Denote by $\left(f_{1}, f_{2}, f_{3}, f_{4}\right)$ the sequence $\varphi$. Then the types of $f_{1}, f_{2}, f_{3}, f_{4}$ are:

$$
\mathrm{II}^{+}(a, b, h), \operatorname{III}(+,-a,-b,-c,-a b h), \operatorname{III}(-, a, b,-c,-a b h), \mathrm{II}^{-}(a, b, h)
$$

if $e=-$,

$$
\mathrm{II}^{+}(a, b, h), \operatorname{III}(-,-a,-c,-b,-a b h), \operatorname{III}(+, a,-c, b,-a b h), \mathrm{II}^{-}(a, b, h)
$$

if $e=0$ and

$$
\mathrm{II}^{+}(a, b, h), \operatorname{III}(+,-c,-a,-b,-a b h), \operatorname{III}(-,-c, a, b,-a b h), \mathrm{II}^{-}(a, b, h)
$$

if $e=+$. The result follows.

4.6 Movie moves of type $\mathbf{M V M}_{5}$. Consider a link in $\mathbf{R}^{3}$ that projects on the plane by an immersion and suppose that every crossing is simple except one and the image of the link looks like the following near the singular crossing:

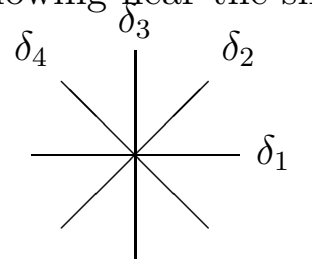

If we move the link a little bit, we get a diagram like:

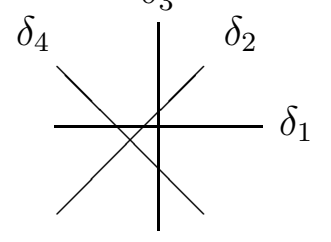

For $\{i, j\} \subset\{1,2,3,4\}$, denote by $x_{i j}$ the intersection of $\delta_{i}$ and $\delta_{j}$. One checks the following fact: if, during an small isotopy, $x_{24}-x_{13}$ makes one counterclockwise turn around $0, x_{14}-x_{23}$ and $x_{34}-x_{12}$ make one clockwise turn around 0 . Therefore any small isotopy such that $x_{i j}-x_{k l}$ makes one turn around 0 is equivalent to an isotopy where $\delta_{3}$ and $\delta_{4}$ are fixed and $\delta_{1}$ and $\delta_{2}$ are moving by translations. During this isotopy, we get diagrams $D_{z}$ where $z=x_{12}$ and $z$ makes one counterclockwise turn around $x=x_{34}$. If $z$ is not in one of the initial $\delta_{i}$ 's, $D_{z}$ is a link diagram. So we get a movie move which is the composite of eight Reidemeister moves of type III.

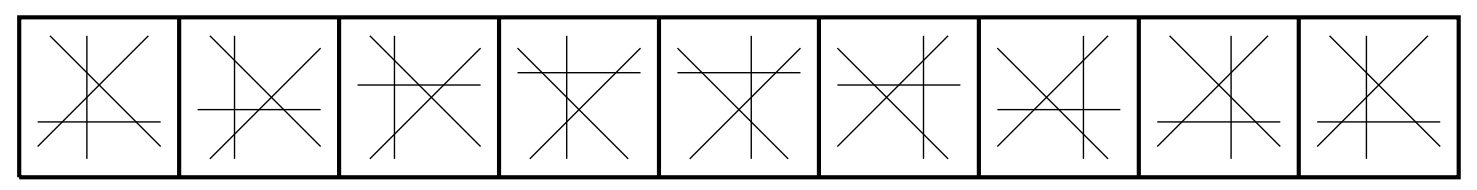

This move depends essentially on the heights $c_{1}, c_{2}, c_{3}, c_{4}$ of the lines $\delta_{1}, \delta_{2}, \delta_{3}, \delta_{4}$. We say that this move is a movie move of type $\operatorname{MVM}_{5}\left(c_{1}, c_{2}, c_{3}, c_{4}\right)$.

4.6.a Lemma: Let $\varphi$ be a movie move of type $M V M_{5}$. Then $\varphi^{0}$ is homotopic to the identity. 
In the oriented case, $\varphi^{1}$ is also homotopic to the identity.

Proof: Consider a movie move $\varphi$ of type $\operatorname{MVM}_{5}\left(c_{1}, c_{2}, c_{3}, c_{4}\right)$. The morphism $\varphi^{0}$ acts on $k h(D)$ for some link diagram $D$. The modification area of $\varphi$ involves six crossings and, to describe $\varphi^{0}$, we have to decompose $k h(D)$ into a direct sum of 64 complexes. So an explicit description of $\varphi^{0}$ is almost impossible, at least by hand. But it is possible to compute $\varphi^{0}$ using a program on a computer and we check that $\varphi^{0}$ is an idempotent (i.e. $\varphi^{0} \circ \varphi^{0}=\varphi^{0}$ ) when the sequence $\left(c_{i}\right)$ is increasing or decreasing. This property is may be related to the following fact which is easy to check: all the Reidemeister moves of $\varphi$ are of type III $_{+}$if $\left(c_{i}\right)$ is increasing and of type III - if $\left(c_{i}\right)$ is decreasing.

Since $\varphi^{0}$ is a homotopy equivalence it has a homotopy inverse $\psi$. So when $\left(c_{1}, c_{2}, c_{3}, c_{4}\right)$ is increasing or decreasing, we have:

$$
\varphi^{0} \sim \psi \circ \varphi^{0} \circ \varphi^{0}=\psi \circ \varphi^{0} \sim \operatorname{Id}
$$

and $\varphi^{0}$ is homotopic to the identity.

In the other cases, we have to proceed differently. Let $S$ be the set of sequences $\left(c_{1}, c_{2}, c_{3}, c_{4}\right)$ of distinct reals such that the lemma is true for every movie move of type $\operatorname{MVM}_{5}\left(c_{1}, c_{2}, c_{3}, c_{4}\right)$. So we have:

$$
c_{1}>c_{2}>c_{3}>c_{4} \quad \Longrightarrow \quad\left(c_{1}, c_{2}, c_{3}, c_{4}\right) \in S
$$

On the other hand, it is easy to see that a movie move of type $\operatorname{MVM}_{5}\left(c_{1}, c_{2}, c_{3}, c_{4}\right)$ is conjugate to a movie move of type $\mathrm{MVM}_{5}\left(c_{2}, c_{3}, c_{4}, c_{1}\right)$. So we have:

$$
\left(c_{1}, c_{2}, c_{3}, c_{4}\right) \in S \Longleftrightarrow\left(c_{2}, c_{3}, c_{4}, c_{1}\right) \in S
$$

Let $c_{1}, c_{2}, c_{3}, c_{4}$ be distinct reals and $\varphi$ be a movie move of type $\operatorname{MVM}_{5}\left(c_{1}, c_{2}, c_{3}, c_{4}\right)$ acting on a diagram $D$. It is possible to modify $D$ by a Reidemeister move of type II in such a way to get the following configuration in $D$ :

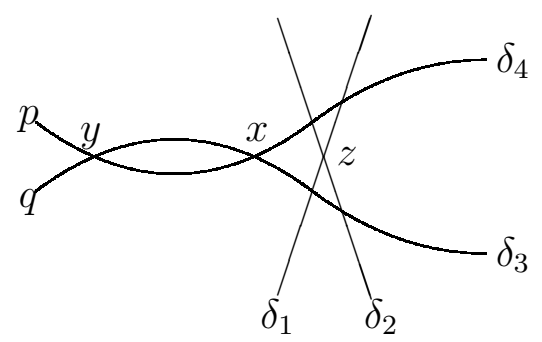

In this figure $\delta_{i}$ have constant height $c_{i}$ and, during the isotopy, $\delta_{3}$ and $\delta_{4}$ are fixed and $\delta_{1}$ and $\delta_{2}$ are moving by translation. So the isotopy of the figure is determined by the isotopy of $z$.

If $z$ makes one counterclockwise turn around $x$, we get a movie move of type $\operatorname{MVM}_{5}\left(c_{1}, c_{2}, c_{3}, c_{4}\right)$. If $z$ makes one counterclockwise turn around $y$, we get a movie move of type $\mathrm{MVM}_{5}\left(c_{1}, c_{2}, c_{4}, c_{3}\right)$. 
Let $\Delta$ be the loop containing $x$ and $y$. Suppose that $\left(c_{1}, c_{2}, c_{4}, c_{3}\right)$ is in $S$. Then the move $\varphi$ of type $\operatorname{MVM}_{5}\left(c_{1}, c_{2}, c_{3}, c_{4}\right)$ is equivalent to the move $\varphi^{\prime}$ obtained by moving $z$ around $\Delta$. So we have:

$$
\varphi^{\prime 0}=D \circ C \circ B \circ A
$$

where $A$ is obtained by moving $\delta_{1}$ through $\Delta, B$ by moving $\delta_{2}$ through $\Delta, C$ by moving back $\delta_{1}$ trough $\Delta$ and $D$ by moving back $\delta_{2}$ through $\Delta$. Denote by $f$ the Reidemeister move of type II which creates crossings $x$ and $y$ and by $g$ its inverse move. So the morphism $\varphi^{0}$ is homotopic to:

$$
f^{0} \circ g^{0} \circ D \circ f^{0} \circ g^{0} \circ C \circ f^{0} \circ g^{0} \circ B \circ f^{0} \circ g^{0} \circ A \circ f^{0} \circ g^{0}
$$

But each morphism $g^{0} \circ X \circ f^{0}$ (with $X=A, B, C, D$ ) is obtained by a type III Reidemeister move and a movie move of type $\mathrm{MVM}_{4}$ : Let $\psi\left(\right.$ resp. $\left.\psi^{\prime}\right)$ be the Reidemeister move obtained by pushing up $z$ through $\delta_{4}$ (resp. pushing down $z$ through $\left.\delta_{3}\right)$. If $a, b, c$ are distinct reals, we set $\mu(a, b, c)=1$ is $a$ is between $b$ and $c$ and $\mu(a, b, c)=-1$ otherwise. So we have:

$$
\begin{gathered}
g^{0} \circ A \circ f^{0} \sim \mu\left(c_{1}, c_{3}, c_{4}\right) T\left(\omega^{-1} \otimes \bar{\omega}\right) \psi \\
g^{0} \circ B \circ f^{0} \sim \mu\left(c_{2}, c_{3}, c_{4}\right) \psi^{-1} T\left(\bar{\omega}^{-1} \otimes \omega\right) \\
g^{0} \circ C \circ f^{0} \sim \mu\left(c_{1}, c_{3}, c_{4}\right) T\left(\bar{\omega} \otimes \omega^{-1}\right) \psi^{\prime} \\
g^{0} \circ D \circ f^{0} \sim \mu\left(c_{2}, c_{3}, c_{4}\right) \psi^{\prime-1} T\left(\omega \otimes \bar{\omega}^{-1}\right)
\end{gathered}
$$

where $T$ is the map $u \otimes v \mapsto T_{p}(u) T_{q}(v)$. Therefore $\varphi^{0}$ is homotopic to the identity and $\left(c_{1}, c_{2}, c_{3}, c_{4}\right)$ belongs to $S$. So we have:

$$
\left(c_{1}, c_{2}, c_{3}, c_{4}\right) \in S \Longleftrightarrow\left(c_{1}, c_{2}, c_{4}, c_{3}\right) \in S
$$

and $S$ contains all sequences $\left(c_{i}\right)$. So the first part of the lemma is proven and the second one follows easily.

Remark: The general formula for $\varphi^{\mathscr{K}}$ is too complicated to be written down if $\varphi$ is a movie move of type $\mathrm{MVM}_{5}$.

4.8 Movie moves of type $\mathrm{MVM}_{6}$. Let $D$ be a link diagram. We can modify $D$ by adding a small circle near a branch of $D$ and then connect the circle to $D$ near a point $p$ in the branch. So we get a movie sequence $\varphi=(f, g)$ where $f$ is a surgery move of index 0 and $g$ a surgery move of index 1 . The second sequence is $\psi=\mathrm{Id}$. The move $(\varphi, \psi)$ is called a movie move of type $\mathrm{MVM}_{6}$ near $p$.

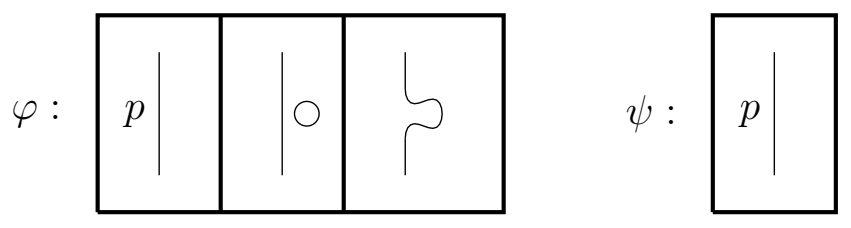


An easy computation gives the following:

4.8.a Lemma: Let $(\varphi, \psi)$ be a movie move of type $M V M_{6}$. Then we have:

$$
\varphi^{0}=\psi^{0} \quad(\bar{\varphi})^{0}=(\bar{\psi})^{0}
$$

In the oriented case, suppose that the first move in $\varphi$ is a surgery move of type $(0, a, h)$. then we have:

$$
\begin{aligned}
\varphi^{1}=\psi^{1} & (\bar{\varphi})^{1}=(\bar{\psi})^{1} \\
\varphi^{\mathscr{K}}=\widehat{T}_{p}(X(a, h) Y(-a, h)) \psi^{\mathscr{K}} & (\bar{\varphi})^{\mathscr{K}}=(\bar{\psi})^{\mathscr{K}} \widehat{T}_{p}(Z(a, h) Y(a,-h))
\end{aligned}
$$

4.9 Movie moves of type $\mathbf{M V M}_{7}$. Let $D$ be a link diagram and $e$ be a sign. We have a movie move $(\varphi, \psi)$ described as follows:
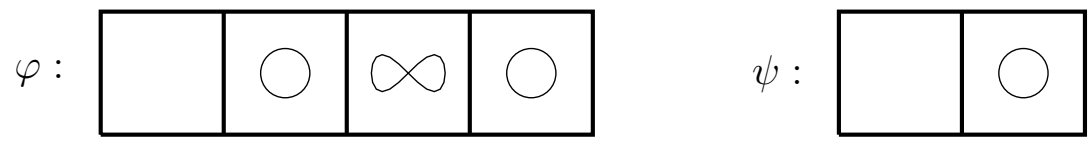

We add a small circle to $D$ by a surgery of index 0 . Then we apply a Reidemeister move of type $\mathrm{I}_{e}^{+}$which creates a loop in the right part of the circle and we remove the other loop in the circle by a Reidemeister move of type $\mathrm{I}_{e}^{-}$. So we get the movie sequence $\varphi$. The movie sequence $\psi$ is a surgery move of index 0 . The move $(\varphi, \psi)$ is called a movie move of type $\mathrm{MVM}_{7}(e)$.

An easy computation gives the following:

4.9.a Lemma: Let $e$ be a sign and $(\varphi, \psi)$ be a movie move of type $\operatorname{MVM}_{7}(e)$. Let $p$ be a point in the created circle. Then we have:

$$
\varphi^{0}=-e T_{p}\left(\frac{\omega^{(e)}}{\bar{\omega}}\right) \psi^{0} \quad(\bar{\varphi})^{0}=e(\bar{\psi})^{0} T_{p}\left(\frac{\omega}{\omega^{(e)}}\right)
$$

In the oriented case, suppose that the first move in $\varphi$ is a surgery move of type $(0, a, h)$. Then we have:

$$
\begin{array}{ccc}
\varphi^{1}=-e \widehat{T}_{p}\left(\frac{\omega^{(-e a h)}}{\omega^{(a h)}}\right) \psi^{1} & (\bar{\varphi})^{1}=e(\bar{\psi})^{1} \widehat{T}_{p}\left(\frac{\omega^{(e a h)}}{\omega^{(a h)}}\right) \\
\varphi^{\mathscr{K}}=\widehat{T}_{p}\left(X(a, h) \frac{A(e,-a, h)}{A(e, a, h)}\right) \varphi^{1} & \psi^{\mathscr{K}}=\widehat{T}_{p}(X(-a, h)) \psi^{1} \\
(\bar{\varphi})^{\mathscr{K}}=(\bar{\varphi})^{1} \widehat{T}_{p}\left(\frac{A(e, a, h)}{A(e,-a, h)} Z(a, h)\right) & (\bar{\psi})^{\mathscr{K}}=(\bar{\psi})^{1} \widehat{T}_{p}(Z(-a, h))
\end{array}
$$

4.10 Movie moves of type $\mathbf{M V M}_{8}$. Let $D$ be a link diagram and $e$ be a sign. We have a movie move $(\varphi, \psi)$ described as follows:
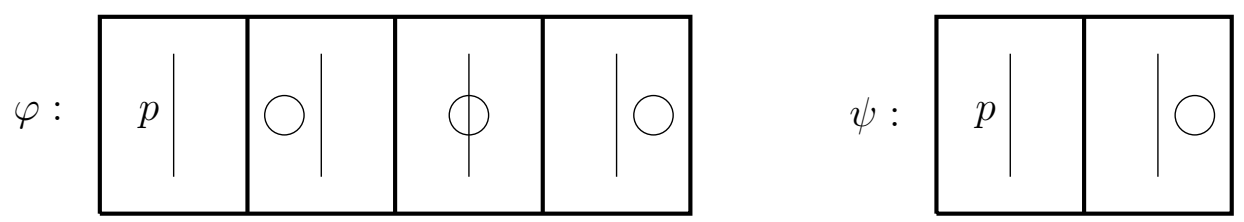
The move $\varphi$ is obtained by creating a small circle of the left hand side of a branch of $D$ with a surgery move of index 0 and then moving this circle through the branch with two Reidemeister moves of type II. The move $\psi$ is a surgery move of index 0 creating the circle on the right hand side of the branch. This move depends on a sign $e$ where $e=+$ if and only if the circle goes over the branch. Let $p$ be a point in the branch. We say that $(\varphi, \psi)$ is a movie move of type $\operatorname{MVM}_{8}(e)$ near $p$.

4.10.a Lemma: Let $D$ be a link diagram and $e$ be a sign. Let $(\varphi, \psi)$ be a movie move of type $\operatorname{MVM}_{8}(e)$ near a point $q \in D$. Let $p$ be a point in the created circle. Then we have:

$$
\varphi^{0}=T_{p}\left(\frac{\omega}{\bar{\omega}}\right) \psi^{0} \quad(\bar{\varphi})^{0}=-(\bar{\psi})^{0}
$$

In the oriented case, suppose that the first move of $\varphi$ is a surgery move of type $(0, a, h)$. Let $b$ be the sign such that $b=+$ if and only if the vertical branch is oriented downward. Then we have:

$$
\begin{array}{cc}
\varphi^{1}=\widehat{T}\left(\frac{\omega^{(a h)}}{\omega^{(-a h)}} \otimes 1\right) \psi^{1} & (\bar{\varphi})^{1}=-(\bar{\psi})^{1} \\
\varphi^{\mathscr{K}}=\widehat{T}\left((X(a, h) \otimes 1) \frac{B^{e}(a, b,-a b h)}{B^{e}(a,-b,-a b h)}\right) \varphi^{1} & \psi^{\mathscr{K}}=\widehat{T}(X(a,-h) \otimes 1) \psi^{1} \\
(\bar{\varphi})^{\mathscr{K}}=(\bar{\varphi})^{1} \widehat{T}\left(\frac{B^{e}(a,-b,-a b h)}{B^{e}(a, b,-a b h)}(Z(a, h) \otimes 1)\right) & (\bar{\psi})^{\mathscr{K}}=\widehat{T}(Z(a,-h) \otimes 1)(\bar{\psi})^{1}
\end{array}
$$

where $\widehat{T}$ is the map $u \otimes v \mapsto \widehat{T}_{p}(u) \widehat{T}_{q}(v)$.

Proof: A straightforward computation gives the desired expressions of $\varphi^{0}$ and $(\bar{\varphi})^{0}$ and therefore the expressions of $\varphi^{1}$ and $(\bar{\varphi})^{1}$.

The orientation of the diagram modified by the first move is described as follows:

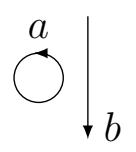

The types of the moves in $\varphi$ are:

$$
(0, a, h), \quad \mathrm{II}^{+}(e, a, b,-a b h), \quad \mathrm{II}^{-}(e, a,-b,-a b h)
$$

and the types of the moves in $\bar{\varphi}$ are:

$$
\mathrm{II}^{+}(e, a,-b,-a b h), \quad \mathrm{II}^{-}(e, a, b,-a b h), \quad(2, a, h)
$$

The result follows.

4.11 Movie moves of type $\mathbf{M V M}_{9}$. Let $D$ be a link diagram and $e$ be a sign. We have a movie move $(\varphi, \psi)$ described as follows:
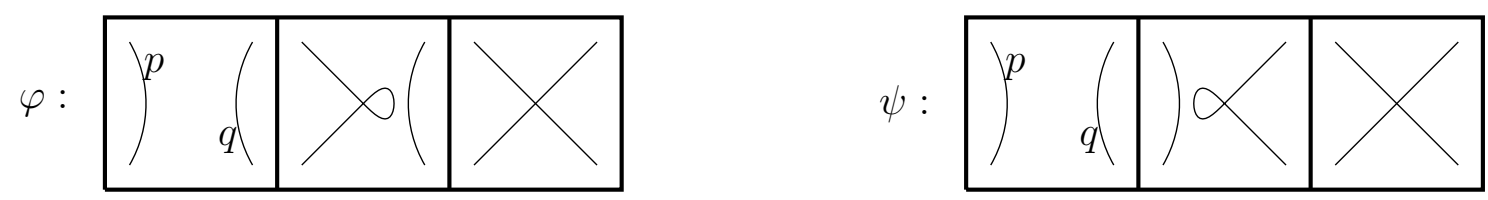
Both moves $\varphi$ and $\psi$ are obtained by a Reidemeister move of type $\mathrm{I}_{e}^{+}$followed by a surgery move of index 1 . We say that $(\varphi, \psi)$ is a movie move of type $\operatorname{MVM}_{9}(e)$ near $(p, q)$.

4.11.a Lemma: Let $D$ be a link diagram and e be a sign. Let $(\varphi, \psi)$ be a movie move of type $\operatorname{MVM}_{9}(e)$ near $(p, q)$. Then we have:

$$
\varphi^{0} T_{p}\left(\frac{\omega^{(e)}}{\bar{\omega}}\right) \sim-e \psi^{0} \quad T_{p}\left(\frac{\omega}{\omega^{(e)}}\right)(\bar{\varphi})^{0} \sim e(\bar{\psi})^{0}
$$

In the oriented case, suppose the first move of $\varphi$ is a Reidemeister move of type $I^{+}(e, a, h)$. Then we have:

$$
\begin{array}{cc}
\varphi^{1} \widehat{T}_{p}\left(\frac{\omega^{(e a h)}}{\omega^{(-a h)}}\right) \sim-e \psi^{1} & \widehat{T}_{p}\left(\frac{\omega^{(a h)}}{\omega^{(e a h)}}\right)(\bar{\varphi})^{1} \sim e(\bar{\psi})^{1} \\
\varphi^{\mathscr{K}} \sim \widehat{T}_{p}(A(e, a, h) Y(-a, h)) \varphi^{1} & \psi^{\mathscr{K}} \sim \widehat{T}_{p}(A(e,-a, h) Y(a, h)) \psi^{1} \\
(\bar{\varphi})^{\mathscr{K}} \sim(\bar{\varphi})^{1} \widehat{T}_{p}\left(\frac{Y(a,-h)}{A(e, a, h)}\right) & (\bar{\psi})^{\mathscr{K}} \sim(\bar{\psi})^{1} \widehat{T}_{p}\left(\frac{Y(-a,-h)}{A(e,-a, h)}\right)
\end{array}
$$

Proof: Set: $\varepsilon=(1+e) / 2$. It is easy to check the following:

$$
\begin{gathered}
\varphi^{0} T_{p}\left(\omega^{\varepsilon}\right)=-e \psi^{0} T_{q}\left(\omega^{\varepsilon}\right) \\
T_{p}\left(\omega^{1-\varepsilon}(\bar{\varphi})^{0}=e T_{q}\left(\omega^{1-\varepsilon}(\bar{\psi})^{0}\right.\right.
\end{gathered}
$$

So we get:

$$
\begin{gathered}
-e \psi^{0}=\varphi^{0} T_{p}\left(\omega^{\varepsilon}\right) T_{q}\left(\omega^{-\varepsilon}\right)=T_{q}\left(\omega^{-\varepsilon}\right) \varphi^{0} T_{p}\left(\omega^{\varepsilon}\right) \sim T_{p}\left(\bar{\omega}^{-\varepsilon}\right) \varphi^{0} T_{p}\left(\omega^{\varepsilon}\right) \\
=\varphi^{0} T_{p}\left(\bar{\omega}^{-\varepsilon} \omega^{\varepsilon}\right)=\varphi^{0} T_{p}\left(\frac{\omega^{(e)}}{\bar{\omega}}\right) \\
e(\bar{\psi})^{0}=T_{q}\left(\omega^{\varepsilon-1}\right) T_{p}\left(\omega^{1-\varepsilon}\right)(\bar{\varphi})^{0}=T_{p}\left(\omega^{1-\varepsilon}\right)(\bar{\varphi})^{0} T_{q}\left(\omega^{\varepsilon-1}\right) \sim T_{p}\left(\omega^{1-\varepsilon}\right)(\bar{\varphi})^{0} T_{p}\left(\bar{\omega}^{\varepsilon-1}\right) \\
=T_{p}\left(\bar{\omega}^{\varepsilon-1} \omega^{1-\varepsilon}\right)(\bar{\varphi})^{0}=T_{p}\left(\frac{\omega}{\bar{\omega}^{(e)}}\right)(\bar{\varphi})^{0}
\end{gathered}
$$

and we get the first formulae and therefore the following:

$$
\varphi^{1} T_{p}\left(\frac{\omega^{(e)}}{\bar{\omega}}\right) \sim-e \psi^{1} \quad T_{p}\left(\frac{\omega}{\omega^{(e)}}\right)(\bar{\varphi})^{1} \sim e(\bar{\psi})^{1}
$$

Since the first move of $\varphi$ is a Reidemeister move of type $\mathrm{I}^{+}(e, a, h)$, the orientation of $D$ is as follows:

$$
\int_{a}^{p} q \downarrow a
$$


and the types of the moves in $\varphi$ (resp. $\psi$ ) are $\mathrm{I}^{+}(e, a, h)$ and $(1,-a, h)$ (resp. $\mathrm{I}^{+}(e,-a, h)$ and $\left.(1, a, h)\right)$. For $\bar{\varphi}($ resp. $\bar{\psi})$, the types are $(1, a,-h)$ and $\mathrm{I}^{-}(e, a, h)$ (resp. $(1,-a,-h)$ and $\left.\mathrm{I}^{-}(e,-a, h)\right)$. The result follows.

4.12 Movie moves of type $\mathbf{M V M}_{10}$. Consider a movie move $(\varphi, \psi)$ acting on a link diagram $D$ as follows:
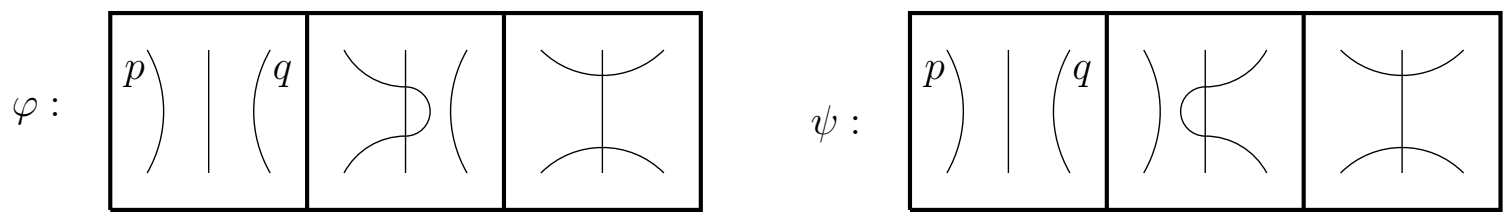

Both moves $\varphi$ and $\psi$ are obtained by a Reidemeister move of type $\mathrm{II}^{+}$followed by a surgery move of index 1 . Let $e$ be the sign such that $e=+$ (resp. $e=-$ ) if the middle branch is under (resp. over) the other ones in the modified diagram. We say that $(\varphi, \psi)$ is a movie move of type $\operatorname{MVM}_{10}(e)$ near $(p, q)$.

4.12.a Lemma: Let $(\varphi, \psi)$ be a movie move of type $\operatorname{MVM}_{10}(e)$ near $(p, q)$. Then we have:

$$
\varphi^{0} T_{p}\left(\frac{\omega}{\bar{\omega}}\right) \sim \psi^{0} \quad(\bar{\varphi})^{0}=-(\bar{\psi})^{0}
$$

In the oriented case, suppose that the first move of $\varphi$ is a Reidemeister move of type $\mathrm{II}^{+}(e, a, b, h)$. Then we have:

$$
\begin{array}{cc}
\varphi^{1} \widehat{T}\left(\frac{\omega^{(b h)}}{\omega^{(-b h)}} \otimes 1\right) \sim \psi^{1} & (\bar{\varphi})^{1}=-(\bar{\psi})^{1} \\
\varphi^{\mathscr{K}} \sim \varphi^{1} \widehat{T}\left(B^{e}(a, b, h)(Y(-a, a b h) \otimes 1)\right) & \psi^{\mathscr{K}} \sim \psi^{1} \widehat{T}\left(B^{e}(a,-b, h)(Y(-a,-a b h) \otimes 1)\right) \\
(\bar{\varphi})^{\mathscr{K}} \sim \widehat{T}\left(\frac{Y(a,-a b h) \otimes 1}{B^{e}(a, b, h)}\right)(\bar{\varphi})^{1} & (\bar{\psi})^{\mathscr{K}} \sim \widehat{T}\left(\frac{Y(a, a b h) \otimes 1}{B^{e}(a,-b, h)}\right)(\bar{\psi})^{1}
\end{array}
$$

where $r$ is a point in the middle branch and $\widehat{T}$ the map $u \otimes v \mapsto \widehat{T}_{p}(u) \widehat{T}_{r}(v)$.

Proof: It is easy to check the following:

$$
\psi^{0}=\varphi^{0} T_{p}(\omega) T_{q}\left(\omega^{-1}\right) \quad(\bar{\varphi})^{0}=-(\bar{\psi})^{0}
$$

and that implies:

$$
\psi^{0}=T_{p}(\omega) T_{q}\left(\omega^{-1}\right) \varphi^{0} \sim T_{p}(\omega) T_{p}\left(\bar{\omega}^{-1}\right) \varphi^{0}=\varphi^{0} T_{p}\left(\omega \bar{\omega}^{-1}\right)
$$

By assumption the diagram $D$ is oriented as follows:

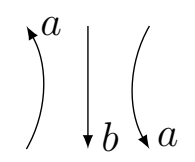

Therefore the types of the moves in $\varphi$ (resp. $\psi$ ) are: 


$$
\mathrm{II}^{+}(e, a, b, h) \text { and }(1, b, a b h)\left(\operatorname{resp} . \mathrm{II}^{+}(e, a,-b, a b h) \text { and }(1,-a,-a b h)\right) .
$$

For the move $\bar{\varphi}$ (resp. $\bar{\psi})$, the types are:

$(1,-b,-a b h)$ and $\mathrm{II}^{-}(e, a, b, h)$ (resp. $(1, a, a b h)$ and $\left.\mathrm{II}^{-}(e, a,-b, a b h)\right)$. The results follows.

4.13 Remark: The conditions induced by all these movie moves are essentially the same as the conditions induced by all the movie moves of Carter and Saito [CS].

Denote by $C(i)$ the condition induced on the correspondance $f \mapsto f^{\mathscr{K}}$ by all the movie moves of type $\mathrm{MVM}_{i}$ and by $C^{\prime}(j)$ the condition induced on it by all the movie moves of type $\mathrm{MM}_{j}$ (by using the Bar Natan classification of these moves [BN2]).

The conditions $C_{j}^{\prime}$ for $j=1,2,3,4,5$ are automatically satisfied here because, for every Reidemeister move $f$ with inverse move $g, g^{\mathscr{K}}$ is a homotopy inverse of $f^{\mathscr{K}}$.

The movie moves of type $\mathrm{MVM}_{1}, \mathrm{MVM}_{2}, \mathrm{MVM}_{4}, \mathrm{MVM}_{5}, \mathrm{MVM}_{6}, \mathrm{MVM}_{9}, \mathrm{MVM}_{10}$ are essentially the movie moves of type $\mathrm{MM}_{7}, \mathrm{MM}_{9}, \mathrm{MM}_{6}, \mathrm{MM}_{10}, \mathrm{MM}_{11}, \mathrm{MM}_{13}$ and $\mathrm{MM}_{15}$. The movie moves of type $\mathrm{MVM}_{7}$ and $\mathrm{MVM}_{8}$ are more or less equivalent to the movie moves of type $\mathrm{MM}_{12}$ and $\mathrm{MM}_{14}$, that is the conditions $C(7)$ and $C(8)$ are exactly the conditions $C^{\prime}(12)$ and $C^{\prime}(14)$. Finally the condition $C(3)$ is equivalent, modulo the conditions $C(0)$ and $C(4)$, to the condition $C^{\prime}(8)$.

\section{Functoriality.}

\subsection{The category of cobordisms of oriented links.}

Denote by $\pi$ the projection map $(x, y, z) \mapsto(x, y)$ from $\mathbf{R}^{3}$ onto $\mathbf{R}^{2}$ and by $\mathscr{E}$ the space of oriented links in $\mathbf{R}^{3}$. We say that a link $L \in \mathscr{E}$ is generic if $\pi$ sends $L$ by an immersion onto a link diagram.

The space $\mathscr{E}$ is a Fréchet manifold and the space $Z \subset \mathscr{E}$ of non generic links is a closed stratified subspace of $\mathscr{E}$.

Let $L_{0}$ and $L_{1}$ be two oriented links. A cobordism $C$ from $L_{0}$ to $L_{1}$ is an oriented compact surface $C$ contained in $\mathbf{R}^{3} \times[0,1]$ and meeting transversally $\mathbf{R}^{3} \times\{0,1\}$ in $\partial C=L_{1} \times\{1\} \cup\left(-L_{0}\right) \times\{0\}$. Two cobordisms $C$ and $C^{\prime}$ from $L_{0}$ to $L_{1}$ are called isotopic if there exist an isotopy $f_{t}: \mathbf{R}^{3} \times[0,1] \rightarrow \mathbf{R}^{3} \times[0,1]$, with $0 \leq t \leq 1$, such that:

$$
\begin{gathered}
f_{0}=\operatorname{Id} \quad f_{1}(C)=C^{\prime} \\
\forall t \in[0,1], \quad f_{t} \text { is the identity on } \mathbf{R}^{3} \times\{0,1\}
\end{gathered}
$$

Let $L_{0}$ and $L_{1}$ be two generic oriented links and $D_{0}$ and $D_{1}$ be the corresponding link diagrams. Let $f: D_{0} \rightarrow D_{1}$ be an elementary move. This move induces a cobordism $C$ from $L_{0}$ to $L_{1}$ which is well defined up to isotopy. Such cobordisms are called elementary cobordisms.

Let $L_{0}$ and $L_{1}$ be two generic oriented links and $C$ be a cobordism from $L_{0}$ to $L_{1}$. We say that $C$ is generic if there is finitely many elements $t_{i} \in(0,1)$ such that: $C \cap \mathbf{R}^{3} \times\{t\}$ is generic for all $t \in[0,1]$ except the $t_{i}$ 's and the cobordism $C \cap \mathbf{R}^{3} \times\left[t_{i}-\varepsilon, t_{i}+\varepsilon\right]$ is elementary for each $i$ and $\varepsilon$ small enough. 
By transversality we see that every $\operatorname{link} L \in \mathscr{E}$ is isotopic to a generic link by a small isotopy. Moreover every cobordism between two generic links in $\mathscr{E}$ is isotopic to a generic cobordism by a small isotopy.

Denote by $\mathscr{L}$ the category of cobordisms of oriented links in $\mathbf{R}^{3}$. The objects of $\mathscr{L}$ is the links in $\mathscr{E}$ and the morphisms are the isotopy classes of cobordisms. Every morphism in $\mathscr{L}$ from a generic link $L_{0}$ to a generic link $L_{1}$ is a composite of elementary morphisms and can be described by a movie sequence.

5.2 Definition: Let $\mathscr{K}$ be a Khovanov data. This data is said to be functorializable if there exists a monoidal functor $\Psi$ from the category of cobordisms of oriented links $\mathscr{L}$ to the homotopy category of $K$-complexes satisfying the following:

- if $D$ is the diagram of a generic oriented link $L$, we have: $\Psi(L)=K H(D)$

- if $C$ is an elementary cobordism from $L_{0}$ to $L_{1}$ associated to an elementary move $f: D_{0} \rightarrow D_{1}, \Psi(C)$ is homotopic to the morphism $f^{\mathscr{K}}$.

If these conditions are satisfied the functor $\Psi$ will be called a Khovanov functor associated to $\mathscr{K}$.

5.3 Lemma: Let $\mathscr{K}$ be a Khovanov data. Suppose $\mathscr{K}$ is functorializable. Then all the Khovanov functors associated to $\mathscr{K}$ are isomorphic.

Proof: Let $\mathscr{L}_{0}$ be the full subcategory of $\mathscr{L}$ generated by generic links. It is clear that the inclusion $\mathscr{L}_{0} \subset \mathscr{L}$ is a equivalence of categories. The result follows.

5.4 Lemma: Let $\mathscr{K}$ be a Khovanov data. Then $\mathscr{K}$ is functorializable if and only if all conditions $C(i)$, for $i=0, \ldots, 10$ are satisfied for the correspondance $f \mapsto f^{\mathscr{K}}$.

Proof: For every movie move $(\varphi, \psi)$, the condition $\varphi^{\mathscr{K}} \sim \psi^{\mathscr{K}}$ will be denoted by $\mathscr{K}(\varphi, \psi)$. It is clear that, if the correspondance $f \mapsto f^{\mathscr{K}}$ comes from a functor, every movie move induces a trivial condition. So all conditions $C(i)$ are satisfied.

Conversely, suppose all conditions $C(i)$, for $i=0, \ldots, 10$ are satisfied. The only thing to do is to define the functor $\Psi$ on the category of cobordisms of generic oriented links $\mathscr{L}_{0}$. This functor is well defined on the objects.

Let $L_{0}$ and $L_{1}$ be two generic oriented links and $C_{0}$ be a cobordism from $L_{0}$ to $L_{1}$. This cobordism represents a morphism $f$ from $L_{0}$ to $L_{1}$. We have to define $\Psi(f)$. Since $C_{0}$ is isotopic to a generic cobordism, $\Psi(f)$ is homotopic to a morphism $\varphi^{\mathscr{K}}$, where $\varphi$ is some movie sequence. So we don't have any choice for $\Psi(f)$. The only thing to do is to prove that $\varphi^{\mathscr{K}}$ depends only on the isotopy class of $C_{0}$.

Let $M=\mathscr{E}\left(C_{0}\right)$ be the space of cobordisms isotopic to $C_{0}$ and $Z_{0}=Z\left(C_{0}\right)$ be the space of cobordisms in $M$ which are not generic. The space $M$ is a connected Fréchet manifold and $Z_{0}$ is a closed stratified subspace of $M$ of codimension 1. Every $C \in M$ which is not in $Z_{0}$ is determined by a movie sequence $\varphi=\widehat{C}$, and $\Psi(C)=\varphi^{\mathscr{K}}$ is well defined. The last thing to do is to prove the following: if $C$ and $C^{\prime}$ are in $M \backslash Z_{0}$, $\Psi(C)$ and $\Psi\left(C^{\prime}\right)$ are homotopic.

Let $C$ be a cobordism in $M$. For every $t \in[0,1]$ denote by $C_{t}$ the intersection $C \cap \mathbf{R}^{3} \times\{t\}$. We say that $t$ is $C$-regular if $C$ is transverse to $\mathbf{R}^{3} \times\{t\}$ and $C_{t}$ is a generic link in $\mathbf{R}^{3} \times\{t\} \simeq \mathbf{R}^{3}$. We say that $t$ is $C$-singular if $t$ is not $C$-regular. 
If $C \in M$ is generic, there is only finitely many $C$-singular elements in $[0,1]$, and every $C$-singular parameter $t$ correspond to a Reidemeister move if $C$ is transverse to $\mathbf{R}^{3} \times\{t\}$ and a surgery move if it is not the case.

Since $M$ is connected, there is a path $\gamma$ in $M$ joining $C$ and $C^{\prime}$. Up to modify $\gamma$ we may as well suppose that $\gamma$ is transverse to $Z_{0}$ and we have to prove that $\Psi(\gamma(s))$ doesn't change when $\gamma(s)$ goes through $Z_{0}$. Let $s_{0}$ be a parameter such that $\gamma\left(s_{0}\right)$ is in $Z_{0}$. Since $\gamma$ is transverse to $Z_{0}, \gamma\left(s_{0}\right)$ is in a stratum $S \subset Z_{0}$ of codimension 1. For $\varepsilon$ small enough, $\gamma\left(s_{0}-\varepsilon\right)$ and $\gamma\left(s_{0}+\varepsilon\right)$ correspond to movie sequences $\varphi$ and $\psi$ and we get a movie move $(\varphi, \psi)$. So every codimension 1 stratum of $Z_{0}$ induces a movie move $(\varphi, \psi)$. The only condition to check is the conditions $\mathscr{K}(\varphi, \psi)$ for all these movie moves.

Let $S$ be a codimension 1 stratum in $Z_{0}$. An element in $S$ is a cobordism $C$ which is not generic but for only one reason. So there is a unique $t_{0} \in(0,1)$ such that $C \cap\left(\mathbf{R}^{3} \times\left[t_{0}-\varepsilon, t_{0}+\varepsilon\right]\right)$ is not generic. Let $(\varphi, \psi)$ be the movie move associated to $C$. The cobordism $C \cap\left(\mathbf{R}^{3} \times\left[t_{0}-\varepsilon, t_{0}+\varepsilon\right]\right)$ induces a movie move $\left(\varphi_{0}, \psi_{0}\right)$ and there are two movies sequences $\alpha$ and $\beta$ such that: $\varphi=\alpha \circ \varphi_{0} \circ \beta$ and $\psi=\alpha \circ \psi_{0} \circ \beta$. So the condition $\mathscr{K}\left(\varphi_{0}, \psi_{0}\right)$ implies the condition $\mathscr{K}(\varphi, \psi)$ and it is enough to consider the case where $t_{0}$ is the only $C$-singular element in $[0,1]$ and $C_{t}$ is generic for every $t \neq t_{0}$.

Suppose $C$ is transverse to $\mathbf{R}^{3} \times\left\{t_{0}\right\}$. Then the problem reduces to an isotopy problem and then to an isotopy corresponding to a loop around a codimension 2 stratum of $Z \subset \mathscr{E}$. These loops correspond to all movie moves of type $\mathrm{MVM}_{i}$ for $0 \leq i \leq 5$ involving only Reidemeister moves.

Suppose $C$ is not transverse to $\mathbf{R}^{3} \times\left\{t_{0}\right\}$. Then the function $C \subset \mathbf{R}^{3} \times[0,1] \rightarrow$ $[0,1]$ has critical points $u_{i}$ is $\mathbf{R}^{3} \times\left\{t_{0}\right\}$. If one of these points is degenerated, the problem reduces to a movie move of type $\mathrm{MVM}_{6}$. If there is at least 2 critical points, the problem reduces to a movie move of type $\mathrm{MVM}_{0}$ involving two surgery moves. In the other cases, we have only one critical point $u$ and this point is non degenerated with index $d$. But we have an extra condition because $C$ belongs to $Z_{0}$. There is two possibilities for this condition: $u$ is a critical point for $\pi: C \rightarrow \mathbf{R}^{2}$ or $\pi(u)$ is a multiple point in $\pi\left(C_{t_{0}}\right)$. In the first case, we get a movie move of type $\mathrm{MVM}_{7}$ if $d=0$ or $d=2$ and a movie move of type $\mathrm{MVM}_{9}$ if $d=1$. In the second case, we get a movie move of type $\mathrm{MVM}_{0}$ involving one Reidemeister move and one surgery move or a movie move of type $\mathrm{MVM}_{8}$ if $d=0$ or $d=2$ and a movie move of type $\mathrm{MVM}_{10}$ if $d=1$.

Thus, if all conditions $C(i)$ are satisfied, the functor $\Psi$ is well defined.

For simplicity we'll identify the sign + with 1 and the sign - with -1 and we define a map $<? \mid$ ? $>$ from $\{ \pm\}^{2}$ to $\{ \pm\}$, a map $\mu$ from $\{ \pm\}$ to $R^{*}$ and a map $\delta$ from $\{ \pm\}^{2}$ to $R^{*}$ by:

$$
\begin{gathered}
<(-1)^{p} \mid(-1)^{q}>=(-1)^{p q} \\
\mu(e)=\omega^{(1+e) / 2}= \begin{cases}\omega & \text { if } e=+ \\
1 & \text { if } e=-\end{cases} \\
\delta(a, b)=\left\{\begin{array}{cc}
\omega \bar{\omega} & \text { if } a=b=+ \\
1 & \text { otherwise }
\end{array}\right.
\end{gathered}
$$


The map $<$ ? $\mid$ ? $>$ is symmetric and satisfy the following property:

$$
\forall a, b, c \in\{ \pm\}, \quad<a|b c>=<a| b><a \mid c>
$$

If $H(h)$ is an element of $R^{*}$ depending on a $\operatorname{sign} h$, we set:

$$
\widetilde{H}=\frac{H(+)}{H(-)}
$$

5.5 Theorem: Let $\mathscr{K}=(A, B, C, X, Y, Z)$ be a Khovanov data. Then $\mathscr{K}$ is functorializable if and only if there exist elements $\sigma(h)$ in $K^{*}, E_{e}(h), F(e)$ in $R^{*}, W(h)$ in $(R \otimes R)^{*}$ depending on signs $e$ and $h$ such that the following holds, for every signs $e, h, a, b, c$ :

$$
\begin{gathered}
\sigma(+) \sigma(-)=\omega \bar{\omega} \\
E_{+}(h) E_{-}(h)=\omega F(+) F(-) U(h) V(h) \\
A(e, a, h)=<e|a><e| h><a \mid h>\left(\frac{\sigma(h)}{\omega^{(a)}}\right)^{(1-e h) / 2} \frac{E_{e}(h)}{F(a)} \\
B(a, b, h)=\frac{<a \mid b>}{\delta(a h, b h)} \sigma(h)^{(1+a b) / 2} U(h) \mu(b h) \otimes V(h) \mu(a h) \\
X(a, h)=<a \mid-h>\left(\frac{\sigma(h)}{\omega^{(a)}}\right)^{(1+h) / 2} F(-a) \\
Y(a, h)=X(-a, h)^{-1} h(a, h)=X(-a,-h) \\
C(e, a, b, c)=-a c\left(1 \otimes\left(\widetilde{E}_{-e}\right)^{-1} H^{(a+b)(b+c) / 4} \otimes 1\right) \widehat{U}^{a(b+c) / 2} \widehat{V}^{(a+b) c / 2}
\end{gathered}
$$

with: $W(h)=U(h) \otimes V(h), H=\widetilde{\sigma}(\widetilde{U} \widetilde{V})^{2}, \widehat{U}=\widetilde{U} \otimes \widetilde{U}^{-1} \otimes 1, \widehat{V}=1 \otimes \widetilde{V}^{-1} \otimes \widetilde{V}$.

Remark: Suppose $\mathscr{K}$ is functorializable. Denote by $\Psi$ the associated functor. The system $(\sigma, E, F, W)$ will be called a parametrization of the Khovanov functor $\Psi$. It is easy to see that such a parametrization is unique.

5.6 Remark: In the classical case, $\omega$ is equal to 1 and the Khovanov data given by:

$$
\begin{gathered}
A(e, a, h)=<e|a><e| h><a|h>\quad B(a, b, h)=<a| b>\quad C(e, a, b, c)=-a c \\
X(a, h)=<a|-h>\quad Y(a, h)=<-a|-h>\quad Z(a, h)=<-a \mid h>
\end{gathered}
$$

is functorializable.

Proof: Because of lemma 4.3.a, the condition $C(2)$ is equivalent to:

$$
\frac{B^{e}(-a,-b, h)}{B^{e}(a, b, h)}=-a b \omega^{(-b h)} \otimes \frac{1}{\omega^{(a h)}}
$$

for every signs $e, a, b, h$. It is easy to see that these conditions are equivalent to:

$$
\frac{B(-a,-b, h)}{B(a, b, h)}=-a b \omega^{(-b h)} \otimes \frac{1}{\omega^{(a h)}}
$$


for every $a, b, h$. Define the elements $B^{\prime}(a, b, h)$ in $(R \otimes R)^{*}$ by:

$$
B(a, b, h)=\frac{<a \mid b>}{\delta(a h, b h)}(\mu(b h) \otimes \mu(a h)) B^{\prime}(a, b, h)
$$

With these new elements, the condition $C(2)$ is equivalent to:

$$
B^{\prime}(-a,-b, h)=B^{\prime}(a, b, h)
$$

and $C(2)$ is equivalent to the fact that $B^{\prime}(a, b, h)$ depends only on $a b$ and $h$ :

$$
B^{\prime}(a, b, h)=B^{\prime \prime}(a b, h)
$$

Because of lemma 4.10.a, the condition $C(8)$ is equivalent to:

$$
\begin{gathered}
\frac{B^{e}(a, b,-a b h)}{B^{e}(a,-b,-a b h)}=\left(\frac{X(a,-h)}{X(a, h)} \frac{\omega^{(-a h)}}{\omega^{(a h)}}\right) \otimes 1 \\
\frac{B^{e}(a, b,-a b h)}{B^{e}(a,-b,-a b h)}=-\left(\frac{Z(a, h)}{Z(a,-h)}\right) \otimes 1
\end{gathered}
$$

These conditions imply the following:

$$
\frac{B(a, b, h)}{B(a,-b, h)} \in R^{*} \otimes 1 \quad \frac{B(a, b, h)}{B(-a, b, h)} \in 1 \otimes R^{*}
$$

and that's equivalent to the fact that

$$
\frac{B^{\prime \prime}(e, h)}{B^{\prime \prime}(-e, h)}
$$

lies in $R^{*} \otimes 1$ and in $1 \otimes R^{*}$ and therefore in $K^{*}(1 \otimes 1)$.

Set:

$$
W(h)=B^{\prime \prime}(-, h) \quad \sigma(h)=B^{\prime \prime}(+, h) W(h)^{-1}
$$

The elements $\sigma(h)$ are in $K^{*}$ and we have:

$$
B(a, b, h)=\frac{<a \mid b>}{\delta(a h, b h)} \sigma(h)^{(1+a b) / 2}(\mu(b h) \otimes \mu(a h)) W(h)
$$

With this expression, we have:

$$
\frac{B^{e}(a, b,-a b h)}{B^{e}(a,-b,-a b h)}=a \sigma(-a b h)^{a b}\left(\omega^{(b h)}\right)^{-a b} \otimes 1
$$

and the condition $C(8)$ is equivalent to:

$$
a \sigma(-a b h)^{a b}\left(\omega^{(b h)}\right)^{-a b}=\frac{X(a,-h)}{X(a, h)} \frac{\omega^{(-a h)}}{\omega^{(a h)}}=-\frac{Z(a, h)}{Z(a,-h)}
$$


So the left hand side term is independant of $b$ and we get: $\sigma(+) \sigma(-)=\omega \bar{\omega}$. Using that, the condition $C(8)$ is equivalent to:

$$
\frac{X(a, h)}{X(a,-h)}=a \frac{\sigma(h)}{\omega^{(a h)}} \quad \frac{Z(a, h)}{Z(a,-h)}=-a \frac{\sigma(-h)}{\omega^{(a h)}}
$$

Set: $F(a)=X(-a,-)$. Then we have:

$$
X(a, h)=<a \mid-h>\left(\frac{\sigma(h)}{\omega^{(a)}}\right)^{(1+h) / 2} F(-a)
$$

The condition $C(6)$ is equivalent to:

$$
X(a, h) Y(-a, h)=1 \quad Z(a, h) Y(a,-h)=1
$$

and $B, X, Y, Z$ can be describe in term of $\sigma, W, F$. Using these descriptions one can check that all conditions $C(i)$, for $i=0,2,6,8,10$, are satisfied.

Define the elements $A^{\prime}(e, a, h)$ by:

$$
A(e, a, h)=<e|a><e a| h>\left(\frac{\sigma(h)}{\omega^{(a)}}\right)^{(1-e h) / 2} \frac{A^{\prime}(e, a, h)}{F(a)}
$$

Using these new elements, the condition $C(7)$ becomes: $A^{\prime}(e, a, h)=A^{\prime}(e,-a, h)$ and $A^{\prime}(e, a, h)$ depends only on $(e, h)$. So by setting: $A^{\prime}(e, a, h)=E_{e}(h)$, we have:

$$
A(e, a, h)=<e|a><e a| h>\left(\frac{\sigma(h)}{\omega^{(a)}}\right)^{(1-e h) / 2} \frac{E_{e}(h)}{F(a)}
$$

Using that, all conditions $C(i)$, for $i=0,2,6,7,8,9,10$, are now satisfied. The condition $C(1)$ becomes the relation:

$$
E_{+}(h) E_{-}(h)=\omega F(+) F(-) U(h) V(h)
$$

with: $U(h) \otimes V(h)=W(h)$ and the last thing to do is to compute the elements $C(e, a, b, c)$ and verify the conditions $C(i)$, for $i=3,4,5$.

Because of lemma 4.4.a, the condition $C(3)$ is equivalent to:

$$
\begin{aligned}
& C(e,-a h,-a h, b h)=u \otimes v \otimes w \Longrightarrow u v \otimes w=a b \widetilde{\sigma}^{(1-a b) / 2} \widetilde{E}_{e} \otimes 1(\widetilde{U} \otimes \widetilde{V})^{-a b} \\
& C(e, b h,-a h,-a h)=u \otimes v \otimes w \Longrightarrow w v \otimes u=a b \widetilde{\sigma}^{(1-a b) / 2} \widetilde{E}_{e} \otimes 1(\widetilde{V} \otimes \widetilde{U})^{-a b}
\end{aligned}
$$

In the case: $b=-a$, we get (with: $C(e,-a h,-a h,-a h)=u \otimes v \otimes w)$ :

$$
\begin{aligned}
& u v \otimes w=-\widetilde{\sigma}\left(\widetilde{E}_{e} \otimes 1\right)(\widetilde{U} \otimes \widetilde{V}) \\
& w v \otimes u=-\widetilde{\sigma}\left(\widetilde{E}_{e} \otimes 1\right)(\widetilde{V} \otimes \widetilde{U})
\end{aligned}
$$

and that implies:

$$
C(e,-a h,-a h,-a h)=-\widetilde{U} \otimes \widetilde{\sigma} \widetilde{E}_{e} \otimes \widetilde{V}
$$


and then:

$$
C(e, a, a, a)=-\widetilde{U} \otimes \widetilde{\sigma} \widetilde{E}_{e} \otimes \widetilde{V}
$$

Consider the condition $C(4)$ given by lemma 4.5.a. This condition depends on signs $a, b, c, h$ and on an element $e$ in $\{+,-, 0\}$. We consider this condition in the case: $a=b=1$.

If $e=-$, this condition is the following:

$$
C(+, h, h, c h) C(-,-h,-h, c h)=-\widetilde{\sigma}(\widetilde{U} \otimes \widetilde{V} \otimes 1)
$$

or:

$$
\begin{gathered}
C(c, c h, c h, c h) C(-c,-c h,-c h, c h)=-\widetilde{\sigma}(\widetilde{U} \otimes \widetilde{V} \otimes 1) \\
\Longrightarrow C(-c,-c h,-c h, c h)=1 \otimes \frac{\widetilde{V}}{\widetilde{E}_{c}} \otimes \frac{1}{\widetilde{V}}
\end{gathered}
$$

So we get:

$$
C(e, a, a,-a)=1 \otimes \frac{\widetilde{V}}{\widetilde{E}_{-e}} \otimes \frac{1}{\widetilde{V}}
$$

If $e=0$ the condition is:

$$
C(+, h, c h, h) C(-,-h, c h,-h)=\widetilde{\sigma}(\widetilde{U} \otimes 1 \otimes \widetilde{V})
$$

or:

$$
\begin{gathered}
C(c, c h, c h, c h) C(-c,-c h, c h,-c h)=\widetilde{\sigma}(\widetilde{U} \otimes 1 \otimes \widetilde{V}) \\
\Longrightarrow C(-c,-c h, c h,-c h)=-1 \otimes \frac{1}{\widetilde{E}_{c}} \otimes 1
\end{gathered}
$$

and we have:

$$
C(e, a,-a, a)=-1 \otimes \frac{1}{\widetilde{E}_{-e}} \otimes 1
$$

If $e=+$ the condition is:

$$
C(+, c h, h, h) C(-, c h,-h,-h)=-\widetilde{\sigma}(1 \otimes \widetilde{U} \otimes \widetilde{V})
$$

or:

$$
\begin{gathered}
C(c, c h, c h, c h) C(-c, c h,-c h,-c h)=-\widetilde{\sigma}(1 \otimes \widetilde{U} \otimes \widetilde{V}) \\
\Longrightarrow C(-c, c h,-c h,-c h)=\frac{1}{\widetilde{U}} \otimes \frac{\widetilde{U}}{\widetilde{E}_{c}} \otimes 1
\end{gathered}
$$

and we have:

$$
C(e,-a, a, a)=\frac{1}{\widetilde{U}} \otimes \frac{\widetilde{U}}{\widetilde{E}_{e}} \otimes 1
$$

So we get the general formula:

$$
C(e, a, b, c)=-a c\left(1 \otimes\left(\widetilde{E}_{-e}\right)^{-1} H^{(a+b)(b+c) / 4} \otimes 1\right) \widehat{U}^{a(b+c) / 2} \widehat{V}^{(a+b) c / 2}
$$

with: $H=\widetilde{\sigma}(\widetilde{U} \widetilde{V})^{2}, \widehat{U}=\widetilde{U} \otimes \widetilde{U}^{-1} \otimes 1, \widehat{V}=1 \otimes \widetilde{V}^{-1} \otimes \widetilde{V}$. 
Using this expression, it is easy to check the conditions $C(3)$ and $C(4)$ and the last condition to check is $C(5)$.

Consider a movie move of type $\operatorname{MVM}_{5}\left(c_{1}, c_{2}, c_{3}, c_{4}\right)$, where the $c_{i}$ 's are distinct reals. This move involves four lines $\delta_{1}, \delta_{2}, \delta_{3}, \delta_{4}$ and each $c_{i}$ is the height of $\delta_{i}$. Since we are consider oriented links, each line has to be oriented. Let $a, b, c, d$ be four signs and suppose the singular link diagram is oriented as follows:

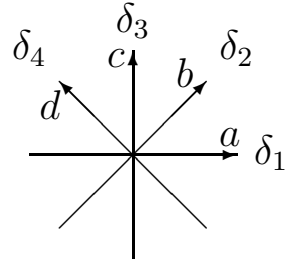

This figure induces a movie move of type $\operatorname{MVM}_{5}\left(c_{1}, c_{2}, c_{3}, c_{4}\right)$ and then an element $M(a, b, c, d, h) \in\left(R^{4}\right)^{*}$ depending on the signs $a, b, c, d$ and the $D$-sign of the center of the triangle corresponding to the first Reidemeister move. So we have to prove that $M(a, b, c, d, h)$ is allways equal to 1 .

First of all, consider the case where the sequence $\left(c_{1}, c_{2}, c_{3}, c_{4}\right)$ is decreasing.

For each $k$ in $\{1,2,3,4\}$ denote by $F_{k}: R^{\otimes 3} \rightarrow R^{\otimes 4}$ the tensorization by 1 at the $k$-th position. For example, $F_{2}$ is the map:

$$
u \otimes v \otimes w \mapsto u \otimes 1 \otimes v \otimes w
$$

We have the following:

$$
\begin{gathered}
M(a, b, c, d, h)=F_{4}\left(C(-, a h,-b h, c h)^{h}\right) F_{3}\left(C(-,-a h, b h,-d h)^{-h}\right) \times \\
F_{2}\left(C(-, a h,-c h, d h)^{h}\right) F_{1}\left(C(-,-b h, c h,-d h)^{-h}\right) \times \\
F_{4}\left(C(-,-a h, b h,-c h)^{h}\right) F_{3}\left(C(-, a h,-b h, d h)^{-h}\right) \times \\
F_{2}\left(C(-,-a h, c h,-d h)^{h}\right) F_{1}\left(C(-, b h,-c h, d h)^{-h}\right)
\end{gathered}
$$

But $C(e, a h, b h, c h)$ doesn't depend on $h$. So we have:

$$
M(a, b, c, d, h)^{h}=M^{2}
$$

with:

$$
M=\frac{F_{4}(C(-, a,-b, c)) F_{2}(C(-, a,-c, d))}{F_{3}(C(-, a,-b, d)) F_{1}(C(-, b,-c, d))}
$$

A straightforward computation gives the following:

$$
\begin{gathered}
M=\left(1 \otimes H^{(a-b)(c-d) / 4} \otimes H^{-(a-b)(c-d) / 4} \otimes 1\right)\left(1 \otimes \widetilde{U}^{-(a-b)(c-d) / 2} \otimes \widetilde{U}^{(a-b)(c-d) / 2} \otimes 1\right) \times \\
\left(1 \otimes \widetilde{V}^{-(a-b)(c-d) / 2} \otimes \widetilde{V}^{(a-b)(c-d) / 2} \otimes 1\right)=1 \otimes X \otimes X^{-1} \otimes 1
\end{gathered}
$$

with:

$$
X=H^{(a-b)(c-d) / 4} \widetilde{U}^{-(a-b)(c-d) / 2} \widetilde{V}^{-(a-b)(c-d) / 2}=\left(H \widetilde{U}^{-2} \widetilde{V}^{-2}\right)^{(a-b)(c-d) / 4}=\widetilde{\sigma}^{(a-b)(c-d) / 4}
$$


Therefore $M$ is equal to 1 and the condition $C(5)$ is allways satisfied when the sequence $\left(c_{i}\right)$ is decreasing. For a general sequence, we use the fact that the condition $C(4)$ is allways satisfied and we check the condition with exactly the same proof as the proof of lemma 4.6.a. So all the conditions $C(i)$ are satisfied and the theorem is proven. Therefore theorem $\mathrm{A}$ is also proven.

Let $(\sigma, E, F, W)$ be a parametrization of a Khovanov functor $\Psi$. Consider the following element in $R^{*}$ :

$$
\pi=-\frac{\omega F(+) F(-)}{\sigma(-)}
$$

This element is called the weight of $\Psi$. By construction, every invertible element in $R$ is the weight of a Khovanov functor. For example the weight of the functor described in remark 5.6 is -1 .

5.7 Proposition: Let $\Psi$ be a Khovanov functor and $\pi$ be its weight. For every integer $p \geq 0$, denote by $\Sigma_{p}$ an unknotted oriented surface of genus $p$ in $\mathbf{R}^{4}$. Then we have:

$$
\begin{aligned}
\Psi\left(\Sigma_{p}\right) & =\varepsilon\left(\delta^{p} \pi^{1-p}\right) \\
\sum_{p \geq 0} x^{p} \Psi\left(\Sigma_{p}\right)=\varepsilon\left(\frac{\pi}{1-x \delta / \pi}\right) & =\frac{\varepsilon(\pi)+x\left(2-\varepsilon(\pi)^{2} u\right)}{1-x \varepsilon(\pi) u+x^{2} u} \in K[[x]]
\end{aligned}
$$

with: $u=-\omega \bar{\omega}(\alpha-\bar{\alpha})^{2} /(\pi \bar{\pi})=\delta \bar{\delta} /(\pi \bar{\pi})$.

5.8 Remark: For $\pi=1$, this formula is exactly the same as the formula in the lemma 1.6. Actually, the right hand side part of this formula is the image of the corresponding part in lemma 1.6 by the morphism sending $\varepsilon(1)$ to $\varepsilon(\pi)$ and $\delta$ to $\delta / \pi$.

Proof: Let $(A, B, C, X, Y, Z)$ be a Khovanov data such that $\Psi$ is the corresponding functor. Let $p \geq 0$ be an integer. An unknotted surface of genus $p$ in $\mathbf{R}^{3} \times[0,1]$ can be describe by the following movie sequence:

$$
\varphi=\left(f, g, g^{\prime}, \ldots, f^{\prime}\right)
$$

where $\left(g, g^{\prime}\right)$ is repeated $p$ times. In this sequence, $f, g, g^{\prime}$ and $f^{\prime}$ are surgery moves of type $(0,-,+),(1,-,-),(1,+,+)$ and $(2,-,+)$. So we get:

$$
\Psi\left(\Sigma_{p}\right)=\varepsilon\left(X(-,+) Z(-,+)(Y(-,-) Y(+,+) \omega(\alpha-\bar{\alpha}))^{p}\right)
$$

But it is easy to check the following:

$$
\begin{gathered}
X(-,+) Z(-,+)=X(-,+) X(+,-)=\pi \\
Y(-,-) Y(+,+)=(X(+,-) X(-,+))^{-1}=\frac{1}{\pi}
\end{gathered}
$$

So we have:

$$
\Psi\left(\Sigma_{p}\right)=\varepsilon\left(\delta^{p} \pi^{1-p}\right)
$$




$$
\sum_{p \geq 0} x^{p} \Psi\left(\Sigma_{p}\right)=\varepsilon\left(\frac{\pi}{1-x \delta / \pi}\right)=\frac{\varepsilon(\pi-x \bar{\delta} \pi / \bar{\pi})}{(1-x \delta / \pi)(1-x \bar{\delta} / \bar{\pi})}=\frac{\varepsilon(\pi-x \bar{\delta} \pi / \bar{\pi})}{1-x \varepsilon(\pi) \sigma \bar{\sigma}+x^{2} \sigma \bar{\sigma}}
$$

with: $\sigma=\delta / \pi$. So we get the desired formula.

5.9 Theorem: Two Khovanov functors with the same weight are isomorphic.

Proof: Consider two Khovanov functors $\Psi$ and $\Psi^{\prime}$ with the same weight $\pi$. They are described by two Khovanov data $(A, B, C, X, Y, Z)$ and $\left(A^{\prime}, B^{\prime}, C^{\prime}, X^{\prime}, Y^{\prime}, Z^{\prime}\right)$. Since these two Khovanov data are functorializable there exist elements $\sigma(h)$ in $K^{*}$, $E_{e}(h), F(e)$ in $R^{*}$ and $W(h)$ in $(R \otimes R)^{*}$ such that:

$$
\begin{gathered}
\sigma(+) \sigma(-)=1 \\
E_{+}(h) E_{-}(h)=F(+) F(-) U(h) V(h) \\
A^{\prime}(e, a, h)=A(e, a, h) \sigma(h)^{(1-e h) / 2} \frac{E_{e}(h)}{F(a)} \\
B^{\prime}(a, b, h)=B(a, b, h) \sigma(h)^{(1+a b) / 2} W(h) \\
X^{\prime}(a, h)=X(a, h) \sigma(h)^{(1+h) / 2} F(-a) \\
C^{\prime}(e, a, b, c)=C(e, a, b, c)\left(1 \otimes \widetilde{E}_{-e}^{-1} H^{(a+b)(b+c) / 4} \otimes 1\right) \widehat{U}^{a(b+c) / 2} \widehat{V}^{(a+b) c / 2}
\end{gathered}
$$

with: $W(h)=U(h) \otimes V(h), H=\widetilde{\sigma}(\widetilde{U} \widetilde{V})^{2}, \widehat{U}=\widetilde{U} \otimes \widetilde{U}^{-1} \otimes 1, \widehat{V}=1 \otimes \widetilde{V}^{-1} \otimes \widetilde{V}$.

Because of the first relation, there exists an element $\sigma \in K^{*}$ with: $\sigma(h)=\sigma^{h}$. So we have:

$$
\begin{gathered}
A^{\prime}(e, a, h)=A(e, a, h) \sigma^{(h-e) / 2} \frac{E_{e}(h)}{F(a)} \\
B^{\prime}(a, b, h)=B(a, b, h) \sigma^{h(1+a b) / 2} W(h) \\
X^{\prime}(a, h)=X(a, h) \sigma^{(1+h) / 2} F(-a) \\
H=(\sigma \widetilde{U} \widetilde{V})^{2}
\end{gathered}
$$

Moreover, since $\Psi$ and $\Psi^{\prime}$ have the same weight, we have also:

$$
F(+) F(-) \sigma=1
$$

So, by setting: $F=F(+)$, we have:

$$
F(e)=F^{e} \sigma^{(e-1) / 2}
$$

Consider elements $x_{\varepsilon}(e, h)$ in $R^{*}$ depending on signs $\varepsilon, e$ and $h$.

Let $D$ be an oriented link diagram and $\widehat{D}$ be the oriented resolution of $D$. Denote by $d(D)$ the winding number of $D$. For each component $\widehat{C}$ of $\widehat{D}$ denote by $g^{\prime}(\widehat{C})$ the $(\widehat{D} \backslash \widehat{C})$-sign of a point in $\widehat{C}$. Denote also by $g(D)$ the sum of all $g^{\prime}(\widehat{C})$. It is clear that $d(D)$ and $g(D)$ are both congruent to the number of components of $\widehat{D} \bmod 2$ and $d(D)-g(D)$ is even. 
For each signs $e$ and $h$, denote by $X(e, h)$ the set of crossings of $D$ with sign $e$ and $D$-sign $h$. If $C$ is a component of $D$, denote by $N_{+}(e, h, C)\left(\right.$ resp. $\left.N_{-}(e, h, C)\right)$ the number of crossing $x$ in $X(e, h)$ such that the over branch (resp. the under branch) containing $x$ is in $C$.

Set:

$$
\left.G(D)=\sigma^{(d(D)-g(D)) / 2}{\underset{C}{\otimes}}_{\left(F^{d(C)}\right.} \prod_{\varepsilon, e, h} x_{\varepsilon}(e, h)^{N_{\varepsilon}(e, h, C)}\right)
$$

Let $D_{0}$ be the set of components of $D$. The element $G(D)$ belongs to $R^{\otimes D_{0}}$ and induces, via the maps $\widehat{T}$, an automorphism $A(D): K H(D) \rightarrow K H(D)$ well defined up to homotopy.

By conjugation with these automorphisms, the functor $\Psi^{\prime}$ is transformed into a new fonctor $\Psi^{\prime \prime}$. So, for each morphism $f: D \rightarrow D^{\prime}$, we have a diagram which is commutative up to homotopy:

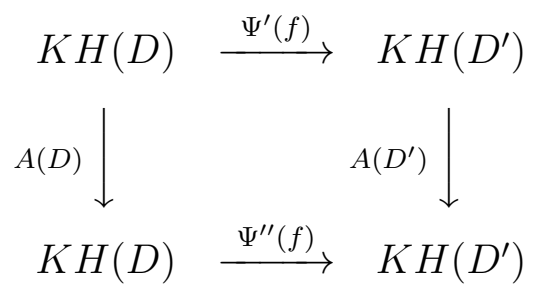

This new functor is clearly isomorphic to $\Psi^{\prime}$. Let's choose the elements $x_{*}(*, *)$ such that:

$$
\begin{gathered}
x_{+}(+, h) x_{+}(-, h)=\frac{1}{U(h)} \quad x_{-}(+, h) x_{-}(-, h)=\frac{1}{V(h)} \\
x_{+}(e, h) x_{-}(e, h)=\frac{\sigma^{(e-1) / 2}}{E_{e}(h)}
\end{gathered}
$$

It is easy to see that this choice is possible. In this case, a straightforward computation shows that $\Psi$ and $\Psi^{\prime \prime}$ agree on every elementary move and therefore on the category $\mathscr{L}_{0}$. Thus $\Psi^{\prime}$ is isomorphic to $\Psi$ and theorem 5.9 (and theorem B) is proven.

5.10 Remark: Consider two isomorphic Khovanov functors of weight $\pi$ and $\pi^{\prime}$. Because of Proposition 5.7, we have: $\varepsilon\left(\pi^{\prime}\right)=\varepsilon(\pi)$. In the case $R=R_{0}$, that implies: $\pi^{\prime}=\pi$ or $\pi^{\prime}=-\theta \bar{\pi}$. So we may ask the question:

Two Khovanov functors of weight $\pi$ and $-\theta \bar{\pi}$ are they isomorphic?

5.11 Khovanov functors and Frobenius endomorphisms. If $f$ is an endomorphism of the Frobenius algebra $R$, it acts on the complexes $K H(D)$ and transforms a Khovanov functor $\Psi$ to a Khovanov functor $\Psi^{\prime}$. Actually, if $(\sigma, E, F, W)$ and $\left(\sigma^{\prime}, E^{\prime}, F^{\prime}, W^{\prime}\right)$ are parametrizations of $\Psi$ and $\Psi^{\prime}$, we have:

$$
\begin{array}{cc}
f(\sigma(h))=\lambda \sigma^{\prime}(h) & f\left(E_{e}(h)\right)=E_{e}^{\prime}(h) \\
f(F(h))=F^{\prime}(h) & f(W(h))=\frac{1}{\lambda} W^{\prime}(h)
\end{array}
$$


where $\lambda$ is the element in $K^{*}$ such that: $f(\omega)=\lambda \omega$.

Therefore, if $\pi$ is the weight of $\Psi$, the weight of $\Psi^{\prime}$ is $f(\pi)$. Another consequence is the fact that there is no Khovanov functor invariant under the endomorphisms of $R$ (at least if $R$ is the universal Frobenius algebra $R_{0}$ ).

5.12 Extensions of Khovanov functors. In section 1.7, a category of mixed cobordisms $\mathscr{C}^{\prime}$ was introduced. This category is a monoidal category containing the category of cobordisms of closed oriented curves $\mathscr{C}$. Moreover the functor associated to $R$ extends to this category. Actually it is possible to define in the same way a category of mixed cobordisms of oriented links $\mathscr{L}^{\prime}$ containing the category $\mathscr{L}$. So we may ask the following:

5.13 Question: Is it possible to extend a Khovanov functor to a monoidal functor from the category $\mathscr{L}^{\prime}$ of mixed cobordisms of oriented links to the homotopy category of $K$-complexes?

If such an extension $\Psi$ exists, the morphism $\Psi(f)$ associated to a mixed cobordism $f$ would be a chain map with a non necessarily zero degree. Notice that, if $L$ is an oriented link and $L^{\prime}$ is the same link but where the orientation of a sublink $L_{1}$ of $L$ is changed, there is a homotopy equivalence from $K H(L)$ to $K H\left(L^{\prime}\right)$ of degree $2 \lambda$, where $\lambda$ is the linking number between $L_{1}$ and $L \backslash L_{1}$.

Actually there is another category $\mathscr{C}^{\prime \prime}$ between $\mathscr{C}$ and $\mathscr{C}^{\prime}$ : the category of decorated cobordisms, where a decorated cobordism is a mixed cobordism on the form $(C, \emptyset, u)$ or equivalently a pair $(C, u)$ where $C$ is a cobordism decorated by a map $u: \pi_{0}(C) \rightarrow R$. Similarly there is a category $\mathscr{L}^{\prime \prime}$ with: $\mathscr{L} \subset \mathscr{L}^{\prime \prime} \subset \mathscr{L}^{\prime}$. Using the operators $\widehat{T}$ it is easy to extend every Khovanov functor to the category $\mathscr{L}^{\prime \prime}$, but the extension to $\mathscr{L}^{\prime}$ is much more problematic.

\section{Invariant of knotted surfaces.}

This section is devoted to the proof of theorem C.

Consider a closed oriented surface $S$ contained in $\mathbf{R}^{4}$. Up to isotopy, we may as well suppose that $S$ is contained in $\mathbf{R}^{3} \times(0,1)$. So this surface is a cobordism in $\mathscr{L}$ from the empty link to itself. Therefore, any Khovanov functor $\Psi$ sends this surface to a morphism from $K$ to $K$ which is the multiplication by an element $\Psi(S) \in K$. Because of proposition 5.7, we have: $\Psi(S)=\varepsilon\left(\delta^{p} \pi^{1-p}\right)$ if $S$ is an unknotted connected surface of genus $p$ and $\pi$ is the weight of $\Psi$. That proves theorem $\mathrm{C}$ if $S$ is unknotted.

In the classical case, the functor $\Psi$ was well defined up to sign and Tanaka [Ta] and Rasmussen [Ra2] proved that $\Psi(S)$ is \pm 2 if $S$ is the torus and 0 if $S$ is any other connected surface. Notice that $\varepsilon\left(\delta^{p}\right)$ in the classical case is equal to 2 if $p=1$ and to 0 otherwise.

Let's say that $(R, \pi)$ is special if the following conditions hold:

- $\delta$ is invertible in $R$

- $\pi$ is a square in $R$

- $R$ has a twisting element which is the square of a element $x \in R$ with: $\bar{x}=x^{-1}$. 
6.1 Lemma: Suppose theorem $C$ is true if $(R, \pi)$ is special. Then the theorem is true in any case.

Proof: Consider a Frobenius algebra $R$ and a Khovanov functor $\Psi$ with weight $\pi \in R^{*}$. Let $R_{1}$ be the following ring:

$$
R_{1}=\mathbf{Z}\left[\alpha, \bar{\alpha}, a, b, c, d,(a+b \alpha)^{-1},(a+b \bar{\alpha})^{-1},(c+d \alpha)^{-1},(c+d \bar{\alpha})^{-1}\right]
$$

This ring is a localization of a polynomial ring with 6 variables. It is equipped with an involution keeping $a, b, c, d$ fixed and exchanging $\alpha$ and $\bar{\alpha}$. Set:

$$
\omega_{1}=a+b \alpha \quad \pi_{1}=c+d \alpha
$$

Then $R_{1}$ is a Frobenius algebra with generator $\alpha$ and twisting element $\omega_{1}$. It is easy to see that there is a unique morphism of Frobenius algebras $f$ from $R_{1}$ to $R$ sending $\alpha, \omega_{1}, \pi_{1}$ to $\alpha, \omega, \pi$ respectively.

Consider the following ring:

$R_{2}=\mathbf{Z}[i]\left[p, q, p_{1}, q_{1}, p_{2}, q_{2}, p^{-1},\left(1+q^{2}\right)^{-1}, 1 / 2, q_{1}^{-1},\left(p_{2}^{2}+q_{2}^{2}\right)^{-1}\right] \subset \mathbf{C}\left(p, q, p_{1}, q_{1}, p_{2}, q_{2}\right)$

The complex conjugation induces an involution on $R_{2}$ and $R_{2}$ is a Frobenius algebra with generator $\left(p_{1}+i q_{1}\right) / p$ and twisting element $p(1+i q)^{2}(1-i q)^{-2}$. It is easy to see that there is a unique morphism $g$ of Frobenius algebras from $R_{1}$ to $R_{2}$ such that:

$$
\begin{gathered}
g(\alpha)=\frac{1}{p}\left(p_{1}+i q_{1}\right) \quad g\left(\omega_{1}\right)=p\left(\frac{1+i q}{1-i q}\right)^{2} \\
g\left(\pi_{1}\right)=\pi_{2}=\left(p_{2}+i q_{2}\right)^{2}
\end{gathered}
$$

It is clear that the fraction field of $R_{2}$ is, via $g$, an algebraic extension of the fraction field of $R_{1}$. Then $g$ is injective.

On the other hand, $R_{2}$ has $i q_{1}$ as generator and the corresponding twisting element is: $\omega_{2}=x^{2}$ with:

$$
x=\frac{1+i q}{1-i q}
$$

and $\left(R_{2}, \pi_{2}\right)$ is special.

Consider a Frobenius functor $\Psi_{1}$ associated with the Frobenius algeba $R_{1}$ with weight $\pi_{1}$. The morphisms $f$ and $g$ send $\Psi_{1}$ to functors $\Psi^{\prime}$ and $\Psi_{2}$ with weight $\pi$ and $\pi_{2}$.

If the theorem is true for special pairs, it is true for $\Psi_{2}$. Since $g: R_{1} \rightarrow R_{2}$ is injective, the theorem is also true for $\Psi_{1}$ and then for $\Psi^{\prime}$. But $\Psi^{\prime}$ is isomorphic to any Khovanov functor with weight $\pi$. Therefore the theorem is true for $\Psi$.

Using this lemma, we may as well suppose that $\Psi$ is a Khovanov functor of weight $\pi$ and that $(R, \pi)$ is special. So $\beta=\alpha-\bar{\alpha}$ is invertible and there exist $x$ and $y$ in $R^{*}$ such that:

$$
\omega=x^{2} \quad x \bar{x}=1 \quad \pi=y^{2}
$$


and we may also suppose that $\Psi$ is parametrized by $(\sigma, E, F, W)$ with:

$$
\sigma(h)=1 \quad E_{e}(h)=\text { ehyx }^{-1} \quad F(a)=-a y x^{-1} \quad W(h)=x^{-1} \otimes x^{-1}
$$

From now on, we will suppose that all these properties are satisfied. The Khovanov data $(A, B, C, X, Y, Z)$ associated to $\Psi$ will be denoted by $\mathscr{K}$.

Consider an oriented link diagram $D$. Let $D^{\circ}$ be the union of $D$ and a trivial circle oriented clockwise and contained in half a plan disjoint from $D$. Let $p$ be a point in this circle. The complex $K H\left(D^{\circ}, p, R\right)$ is naturally isomorphic to $R \otimes_{K} K H(D, R)$ and the graded module $E\left(D^{\circ}, p, R\right)$ is naturally isomorphic to $E(D, R)$. Denote by $\varphi_{D}$ the composite map:

$$
R \otimes_{K} K H(D, R) \stackrel{\sim}{\longrightarrow} K H\left(D^{\circ}, p, R\right) \stackrel{\varphi(R)}{\longrightarrow} E\left(D^{\circ}, p, R\right) \stackrel{\sim}{\longrightarrow} E(D, R)
$$

Because of theorem 2.15, this map is a homotopy equivalence and we have an explicit homotopy inverse of it (see remarks 2.14 and 2.16).

Then for every elementary move $f: D \rightarrow D^{\prime}$ (compatible with the orientations) the morphism $f^{\mathscr{K}}: K H(D) \rightarrow K H\left(D^{\prime}\right)$ induces a well defined $R$-linear map $\widehat{f}$ : $E(D, R) \rightarrow E\left(D^{\prime}, R\right)$. Since $\mathscr{K}$ is functorializable the correspondance $f \mapsto \widehat{f}$ extends to composite of elementary moves (i.e. to movie sequences).

Consider an oriented curve $\Gamma$ in the plane and a component $C$ of $\Gamma$. We set:

$$
\lambda(\Gamma, C)=\omega^{a(1+h) / 2}<a \mid h>
$$

where $a$ is the winding number of $C$ and $h$ is the $(\Gamma \backslash C)$-sign of any point in $C$.

If $D$ is an oriented link diagram, we set:

$$
g(D)=\beta^{(m+q-n) / 2} \prod_{C} \lambda(\widetilde{D}, C)
$$

where the product holds for every component $C$ of the oriented resolution $\widetilde{D}$ of $D$, $q$ being the algebraic number of crossings of $D$ and $m$ (resp. $n$ ) the number of components of $\widetilde{D}$ (resp. $D)$.

Consider an elementary move $f: D \rightarrow D^{\prime}$ between oriented diagrams. Denote by $\pi_{0}(D)$ the set of components of $D$ and by $\widehat{D}$ the set of maps from $\pi_{0}(D)$ to $\{ \pm\}$. Such a map is called a $D$-state. For each state $\sigma \in \widehat{D}$, denote by $Y(\sigma)$ the set of crossings of $D$ between two components $c_{1}$ and $c_{2}$ with $\sigma\left(c_{1}\right) \neq \sigma\left(c_{2}\right)$, by $\widetilde{D}(\sigma)$ the oriented resolution of $D(\sigma)$ and by $d(\sigma)$ the number of components of $\widetilde{D}(\sigma)$. By using the same notations with the diagram $D^{\prime}$, we have sets $\pi_{0}\left(D^{\prime}\right)$ and $\widehat{D}^{\prime}$ and, for each $\sigma \in \widehat{D}^{\prime}$, a set $Y^{\prime}(\sigma)$, a diagram $\widetilde{D}^{\prime}(\sigma)$ and an integer $d^{\prime}(\sigma)$.

This elementary move corresponds to a cobordism $C$ between links associated to $D$ and $D^{\prime}$. So we have two maps from $\widehat{C}$ to $\widehat{D}$ and $\widehat{D}^{\prime}$, where $\widehat{C}$ is the set of $C$-states that is the set of maps from $\pi_{0}(C)$ to $\{ \pm\}$. Let $\sigma$ (resp. $\sigma^{\prime}$ ) be a $D$-state (resp. a $D^{\prime}$-state). We say that $\sigma$ and $\sigma^{\prime}$ are compatible (or: $\sigma \sim \sigma^{\prime}$ ) if they are coming from a state of $C$. A straightforward computation shows the following: 
6.2 Lemma: Let $f: D \rightarrow D^{\prime}$ and $\sigma$ (resp. $\sigma^{\prime}$ ) be a $D$-state (resp. a $D^{\prime}$-state). Suppose that $\sigma$ and $\sigma^{\prime}$ aren't compatible. Then the morphism:

$$
\Lambda^{-e}(Y(\sigma)) \otimes R v(\sigma) \rightarrow E(D, R) \stackrel{\widehat{f}}{\longrightarrow} E\left(D^{\prime}, R\right) \rightarrow \Lambda^{-e}\left(Y^{\prime}\left(\sigma^{\prime}\right)\right) \otimes R v\left(\sigma^{\prime}\right)
$$

is trivial.

An immediate consequence of this lemma is the following:

6.3 Lemma: Let $f: D \rightarrow D^{\prime}$ be a movie sequence represented by a cobordism $C$. Let $\sigma$ and $\sigma^{\prime}$ be a $D$-state and a $D^{\prime}$-state. Suppose the composite map:

$$
\Lambda^{-e}(Y(\sigma)) \otimes R v(\sigma) \rightarrow E(D, R) \stackrel{\widehat{f}}{\longrightarrow} E\left(D^{\prime}, R\right) \rightarrow \Lambda^{-e}\left(Y^{\prime}\left(\sigma^{\prime}\right)\right) \otimes R v\left(\sigma^{\prime}\right)
$$

is not trivial. Then the two states $\sigma$ and $\sigma^{\prime}$ are coming from a $C$-state.

Let $f: D \rightarrow D^{\prime}$ be a movie sequence represented by a cobordism $C$ and $\tau$ be a $C$-state. This state restricts to a $D$-state $\sigma$ and a $D^{\prime}$-state $\sigma^{\prime}$. Define the map $\widehat{f}(\tau)$ by:

$$
\widehat{f}(\tau)=p r^{\prime} \circ \widehat{f} \circ p r
$$

where $p r$ (resp. $p r^{\prime}$ ) is the projection $E(D, R) \rightarrow \Lambda^{-e}(Y(\sigma)) \otimes R v(\sigma) \subset E(D, R)$ (resp. $\left.E\left(D^{\prime}, R\right) \rightarrow \Lambda^{-e}\left(Y^{\prime}\left(\sigma^{\prime}\right)\right) \otimes R v\left(\sigma^{\prime}\right) \subset E\left(D^{\prime}, R\right)\right)$. The map $\widehat{f}(\tau)$ vanishes on $\Lambda^{-e}\left(Y\left(\sigma_{1}\right)\right) \otimes R v\left(\sigma_{1}\right)$ for $\sigma_{1} \neq \sigma$ and is the composite:

$$
\Lambda^{-e}(Y(\sigma)) \otimes R v(\sigma) \rightarrow E(D, R) \stackrel{\widehat{f}}{\longrightarrow} E\left(D^{\prime}, R\right) \rightarrow \Lambda^{-e}\left(Y^{\prime}\left(\sigma^{\prime}\right)\right) \otimes R v\left(\sigma^{\prime}\right) \subset E\left(D^{\prime}, R\right)
$$

on $\Lambda^{-e}(Y(\sigma)) \otimes R v(\sigma)$.

We have clearly the following:

$$
\widehat{f}=\sum_{\tau} \widehat{f}(\tau)
$$

If $f$ corresponds to a closed surface $S, \widehat{f}(\tau)$ is the multiplication by an element $\widehat{S}(\tau) \in R$. Clearly $\Psi(S)$ is the sum of all $\widehat{S}(\tau)$.

Consider now an elementary move $f: D \rightarrow D^{\prime}$ corresponding to a cobordism $C$. Consider a $C$-state $\tau$. This state restricts to a $D$-state $\sigma$ and a $D^{\prime}$-state $\sigma^{\prime}$. A straightforward computation, case by case, gives the following:

6.4 Lemma: Suppose $f$ is a Reidemeister move of type $I^{+}(e, a, h)$. Denote by $c$ the component of $D$ which is modified by $f$. Then the morphism $\widehat{f}(\tau)$ is the map:

$$
u \otimes v(\sigma) \mapsto u \otimes w v\left(\sigma^{\prime}\right)
$$

with:

$$
w=\left(\frac{g\left(D^{\prime}\right)}{g(D)}\right)^{(\sigma(c))}=\frac{g\left(D^{\prime}\left(\sigma^{\prime}\right)\right)}{g(D(\sigma))}<\sigma(c) \mid-e h>
$$


6.5 Lemma: Suppose $f$ is a Reidemeister move of type $I^{+}(a, b, h)$. Let $x_{+}$and $x_{-}$ be the created crossings with sign + and - . Denote by $c_{+}$(resp. $\left.c_{-}\right)$the component of $D$ containing the over (resp. under) branch of the move. Then the morphism $\widehat{f}(\tau)$ is the map:

$$
u \otimes v(\sigma) \mapsto u \otimes w v\left(\sigma^{\prime}\right)
$$

with:

$$
w=\left(\frac{g\left(D^{\prime}\right)}{g(D)}\right)^{\left(\sigma\left(c_{+}\right)\right)}=\frac{g\left(D^{\prime}\left(\sigma^{\prime}\right)\right)}{g(D(\sigma))}<\sigma\left(c_{+}\right) \mid-a b>
$$

if $\sigma\left(c_{+}\right)=\sigma\left(c_{-}\right)$and the map:

$$
u \otimes v(\sigma) \mapsto x_{+} \wedge x_{-} \wedge u \otimes w v\left(\sigma^{\prime}\right)
$$

with:

$w=\frac{g\left(D^{\prime}\left(\sigma^{\prime}\right)\right)}{g(D(\sigma))}<\sigma\left(c_{+}\right)\left|b><\sigma\left(c_{-}\right)\right| a><\sigma\left(c_{+}\right) \mid \sigma\left(c_{-}\right)>x^{-h\left(a \sigma\left(c_{+}\right)+b \sigma\left(c_{-}\right)\right)\left(1-<\sigma\left(c_{+}\right) \sigma\left(c_{-}\right) \mid a b>\right)}$

otherwise.

6.6 Lemma: Suppose $f$ is a Reidemeister move of type $I I I(e, a, b, c, h)$. Let $c_{1}$ (resp. $c_{2}, c_{3}$ ) be the over branch (resp. the middle branch, the under branch) of the move. Then the morphism $\widehat{f}(\tau)$ is the map:

$$
u \otimes v(\sigma) \mapsto u \otimes w v\left(\sigma^{\prime}\right)
$$

with:

$$
w=\left(\frac{g\left(D^{\prime}\right)}{g(D)}\right)^{\left(\sigma\left(c_{1}\right)\right)}=\frac{g\left(D^{\prime}\left(\sigma^{\prime}\right)\right)}{g(D(\sigma))}
$$

if $\sigma\left(c_{1}\right)=\sigma\left(c_{2}\right)=\sigma\left(c_{3}\right)$, and:

$$
w=\frac{g\left(D^{\prime}\left(\sigma^{\prime}\right)\right)}{g(D(\sigma))} \sigma\left(c_{1}\right) \sigma\left(c_{3}\right)
$$

in the general case.

6.7 Lemma: Suppose $f$ is a surgery move of type $(0, a, h)$. Let $c$ be the created circle. Then the morphism $\widehat{f}(\tau)$ is the map:

$$
u \otimes v(\sigma) \mapsto u \otimes w v\left(\sigma^{\prime}\right)
$$

with:

$$
w=\left(\frac{g\left(D^{\prime}\right)}{g(D)} y x^{-1}\right)^{\left(\sigma^{\prime}(c)\right)}=\frac{g\left(D^{\prime}\left(\sigma^{\prime}\right)\right)}{g(D(\sigma))}\left(y x^{-1}\right)^{\left(\sigma^{\prime}(c)\right)}
$$

6.8 Lemma: Suppose $f$ is a surgery move of type $(1, a, h)$. Let $c$ be the component of the cobordism containing the modified branches. Let: $e=1$ (resp. $e=0$ ) if the 
surgery increases (resp. decreases) the number of components of the link. Then the morphism $\widehat{f}(\tau)$ is the map:

$$
u \otimes v(\sigma) \mapsto u \otimes w v\left(\sigma^{\prime}\right)
$$

with:

$$
w=\left(\frac{g\left(D^{\prime}\right)}{g(D)} \beta^{e} x y^{-1}\right)^{(\tau(c))}=\frac{g\left(D^{\prime}\left(\sigma^{\prime}\right)\right)}{g(D(\sigma))} \beta^{e}\left(x y^{-1}\right)^{(\tau(c))}<\tau(c) \mid h>
$$

6.9 Lemma: Suppose $f$ is a surgery move of type $(2, a, h)$. Let $c$ be the component removed by the surgery. Then the morphism $\widehat{f}(\tau)$ is the map:

$$
u \otimes v(\sigma) \mapsto u \otimes w v\left(\sigma^{\prime}\right)
$$

with:

$$
w=\left(\frac{g\left(D^{\prime}\right)}{g(D)} \beta^{-1} y x^{-1}\right)^{(\sigma(c))}=\frac{g\left(D^{\prime}\left(\sigma^{\prime}\right)\right)}{g(D(\sigma))} \beta^{-1}\left(x y^{-1}\right)^{(\sigma(c))}<\sigma(c) \mid-h>
$$

An easy consequence of these lemmas is the following:

6.10 Lemma: Let $f: D \rightarrow D^{\prime}$ be a movie sequence corresponding to a cobordism $C$. Let $\tau$ be a constant $C$-state sending each component of $C$ to a sign $e$. Let $n_{0}$ (resp. $n_{2}$ ) be the number of surgery moves in $f$ of index 0 (resp. 2). Let $n_{1}^{+}$(resp. $n_{1}^{-}$) be the number of surgery moves in $f$ of index 1 which increases (resp. decreases) the number of components of the link. Then the morphism $\widehat{f}(\tau)$ is the map:

$$
u \otimes v(\sigma) \mapsto u \otimes w^{(e)} v\left(\sigma^{\prime}\right)
$$

with:

$$
w=\frac{g\left(D^{\prime}\right)}{g(D)} \beta^{n_{1}^{+}-n_{2}}\left(y x^{-1}\right)^{n_{0}+n_{2}-n_{1}^{+}-n_{1}^{-}}
$$

6.11 Corollary: Let $S$ be a surface in $\mathbf{R}^{4}$. Let $p_{i}$ be the genus of the $i$-th component of $S$. Let $\tau$ be a constant $S$-state sending each component of $S$ to a sign $e$. Then we have:

$$
\widehat{S}(\tau)=\left(\prod_{i}\left(\frac{\delta}{\pi}\right)^{p_{i}-1}\right)^{(e)}
$$

Proof: Let $k$ be the number of components of $S$ and $p$ be the sum of the $p_{i}$ 's. Numbers $n_{0}, n_{1}^{+}, n_{1}^{-}, n_{2}$ are related with a handle decomposition of $S$ and there exist two integers $a, b \geq 0$ such that:

$$
n_{0}=k+a \quad n_{1}^{+}=p+b \quad n_{1}^{-}=p+a \quad n_{2}=k+b
$$


So we have:

$$
\beta^{n_{1}^{+}-n_{2}}\left(y x^{-1}\right)^{n_{0}+n_{2}-n_{1}^{+}-n_{1}^{-}}=\beta^{q}\left(x y^{-1}\right)^{2 q}=\left(\beta x^{2} y^{-2}\right)^{q}=(\delta / \pi)^{q}
$$

with $q=p-k=\sum_{i}\left(p_{i}-1\right)$. The result follows.

Let $f: D \rightarrow D^{\prime}$ be a movie sequence corresponding to a cobordism $C$ and $\tau$ be a $C$-state. Define the $\operatorname{sign} s(f, \tau)$ by the following:

Suppose $f$ is a Reidemeister mode of type III. Denote by $c_{1}$ (resp. $c_{2}, c_{3}$ ) the component of $C$ containing the top branch (resp. the middle branch, the bottom banch) of the move. In this case we set: $s(f, \tau)=-1$ if $\tau\left(c_{1}\right)=\tau\left(c_{3}\right)=-\tau\left(c_{2}\right)$ and $s(f, \tau)=1$ otherwise.

If $f$ is another elementary move we set: $s(f, \tau)=1$.

If $f$ is a movie sequence: $f=\left(f_{1}, f_{2}, \ldots, f_{p}\right)$, we set: $s(f, \tau)=\prod_{i} s\left(f_{i}, \tau\right)$.

If $S$ is a closed oriented surface in $\mathbf{R}^{4}, S$ corresponds to a movie sequence $f$ and we set: $s(S, \tau)=s(f, \tau)$.

6.12 Lemma: Let $S$ be a surface in $\mathbf{R}^{4}$ and $\tau$ be a $S$-state. Let $S_{i}$ be the $i$-th component of $S, p_{i}$ be the genus of $S_{i}$ and $e_{i}$ be the sign $\tau\left(S_{i}\right)$. Then we have:

$$
\widehat{S}(\tau)=s(S, \tau) \prod_{i}\left(\left(\frac{\delta}{\pi}\right)^{p_{i}-1}\right)^{\left(e_{i}\right)}
$$

Proof: Denote by $S_{+}$(resp. $S_{-}$) the submanifold of $S$ where $\tau$ is equal to + (resp. -). By moving down $S_{-}$along the vertical axis in $\mathbf{R}^{3}$, we get a new surface $S^{\prime}$ which is isotopic to $S_{+} \coprod S_{-}$. The $S$-state $\tau$ induces a $S^{\prime}$-state $\tau^{\prime}$. Because of the corollary we have:

$$
\widehat{S}^{\prime}\left(\tau^{\prime}\right)=\widehat{S}_{+}(+) \widehat{S}_{-}(-)=\prod_{i}\left(\left(\frac{\delta}{\pi}\right)^{p_{i}-1}\right)^{\left(e_{i}\right)}
$$

Suppose the morphism $f$ corresponding to $S$ is a movie sequence: $f=\left(f_{1}, \ldots, f_{k}\right)$ where $f_{i}$ is an elementary move from a diagram $D_{i-1}$ to a diagram $D_{i}$. Then the morphism corresponding to $S^{\prime}$ is a movie sequence: $f^{\prime}=\left(f_{1}^{\prime}, f_{2}^{\prime}, \ldots, f_{k}^{\prime}\right)$ where $f_{i}^{\prime}$ is an elementary move from a diagram $D_{i-1}^{\prime}$ to a diagram $D_{i}^{\prime}$. For every $i=1,2, \ldots, k$ we have:

$$
\begin{aligned}
\widehat{f}_{i}(\tau) & =\frac{g\left(D_{i}\left(\sigma_{i}\right)\right)}{g\left(D_{i-1}\left(\sigma_{i-1}\right)\right)} \varphi_{i}(\tau) \\
\widehat{f}_{i}^{\prime}\left(\tau^{\prime}\right) & =\frac{g\left(D_{i}^{\prime}\left(\sigma_{i}^{\prime}\right)\right)}{g\left(D_{i-1}^{\prime}\left(\sigma_{i-1}^{\prime}\right)\right)} \varphi_{i}^{\prime}\left(\tau^{\prime}\right)
\end{aligned}
$$

Using lemmas 6.4 to 6.9 , we check that $\varphi_{i}(\tau)$ and $\varphi_{i}^{\prime}\left(\tau^{\prime}\right)$ are allways the same except for type III Reidemeister moves. In these cases, we have:

$$
\varphi_{i}^{\prime}\left(\tau^{\prime}\right)=s\left(f_{i}, \tau\right) \varphi_{i}(\tau)
$$

Thus we have:

$$
\widehat{S}(\tau)=\prod_{i} \varphi_{i}(\tau)=\prod_{i} s\left(f_{i}, \tau\right) \varphi_{i}^{\prime}\left(\tau^{\prime}\right)=s(f, \tau) \prod_{i} \varphi_{i}^{\prime}\left(\tau^{\prime}\right)=s(S, \tau) \widehat{S}^{\prime}\left(\tau^{\prime}\right)
$$


and the result follows.

6.13 Lemma: Let $S$ be a closed oriented surface in $\mathbf{R}^{4}$ and $\tau$ be a $S$-state. Then we have:

$$
s(S, \tau)=1
$$

Proof: Because of lemma $6.12, s(S, \tau)$ is invariant under isotopy. But $s(S, \tau)$ is also invariant under surgery moves. Therefore $s(S, \tau)$ depends only on the cobordism class of $(S, \tau)$ in the group $\Omega$ of cobordisms of bicolored surfaces in $\mathbf{R}^{4}$. The PontryaginThom construction implies:

$$
\Omega=\pi_{4}\left(M S O_{2} \vee M S O_{2}\right)=\pi_{4}\left(M U_{1} \vee M U_{1}\right)=\pi_{4}\left(B U_{1} \vee B U_{1}\right)
$$

Denote by $E$ the space of paths in $B U_{1}$ ending at the base point. The homotopy fiber $F$ of the inclusion $B U_{1} \vee B U_{1} \subset B U_{1} \times B U_{1}$ is the following:

$$
F=E \times \Omega B U_{1} \underset{\Omega B U_{1} \times \Omega B U_{1}}{\cup} \Omega B U_{1} \times E
$$

But we have a homotopy equivalence: $\left(E, \Omega B U_{1}\right) \sim\left(B^{2}, S^{1}\right)$. So we get a homotopy equivalence:

$$
F \sim B^{2} \times S^{1} \underset{S^{1} \times S^{1}}{\cup} S^{1} \times B^{2}=S^{3}
$$

and we have:

$$
\Omega \simeq \pi_{4}(F) \simeq \pi_{4}\left(S^{3}\right) \simeq \mathrm{Z} / 2
$$

Since $s(S, \tau)$ depends only on the class of $(S, \tau)$ in $\Omega$, we have a map $\varphi: \Omega \rightarrow\{ \pm\}$ such that:

$$
s(S, \tau)=\varphi([S, \tau])
$$

where $[S, \tau]$ is the cobordism class of $(S, \tau)$. By testing this formula for the empty surface we get: $\varphi(0)=1$. Then the last thing to do is to determine $s(S, \tau)$ for some bicolored surface which is not trivial in $\Omega$. Following [CKSS], the non zero element in $\Omega$ is represented by two tori $T_{+}$and $T_{-}$where $T_{+}$intersects $\mathbf{R}^{3} \times\{0\}$ in a Hopf link $H$ and $T_{-}$is the boundary of a regular neighborough of one component of $H$ in $\mathbf{R}^{3} \times\{0\}$.

Consider a movie sequence $f$ from the empty diagram to the diagram $D$ of a Hopf link, given by a 0-surgery, two Reidemeister moves of type $\mathrm{I}_{+}^{+}$and a 1-surgery. Denote by $\bar{f}$ the inverse move. So $T_{+}$is represented by the movie move $(f, \bar{f})$. Consider a movie sequence $f_{1}$ from $D$ to a diagram $D_{1}$ given by a 0 surgery, two Reidemeister moves of type $\mathrm{II}^{+}$and a 1-surgery. This movie creates two circles $C_{1}$ and $C_{2}$ in a neighborough of a component $C$ of the diagram $D$. By moving $C_{1}$ around $C$, this circle goes through the other component of $D$ and we have a movie sequence $f_{2}$ from $D_{1}$ to a diagram $D_{2}$ given by a Reidemeister move of type $\mathrm{II}^{+}$, two Reidemeister moves of type III and a Reidemeister move of type $\mathrm{II}^{-}$. By moving $C_{2}$ around the other part of $C$, we get a movie sequence $f_{3}$ from $D_{2}$ to $D_{3}$ given also by a Reidemeister move of type $\mathrm{II}^{+}$, two Reidemeister moves of type III and a Reidemeister move of 
type $\mathrm{II}^{-}$. Finally we have a movie sequence $f_{4}$ from $D_{3}$ to $D$ given by a 1 -surgery, two Reidemeister moves of type $\mathrm{II}^{-}$and a 2-surgery. The diagrams $D, D_{1}, D_{2}$ and $D_{3}$ are the following:
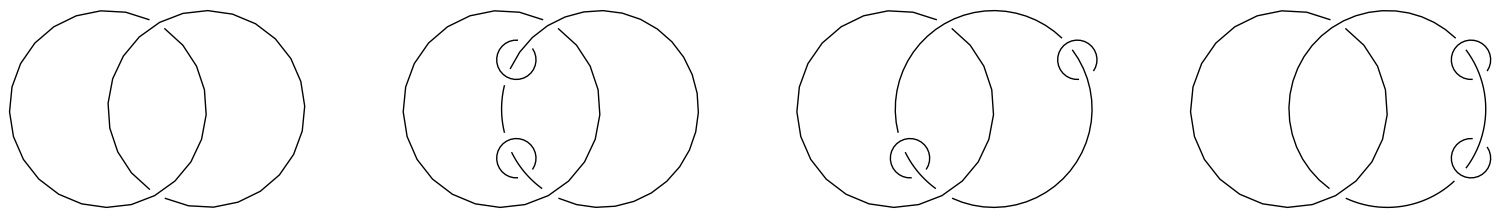

The movie sequence $\left(f, f_{1}, f_{2}, f_{3}, f_{4}, \bar{f}\right)$ represents a bicolored surface $S$ which is not zero in $\Omega$. On the other hand, among the movies $f, \bar{f}$ and the $f_{i}$, only $f_{2}$ and $f_{3}$ contains some type III Reidemeister moves. So we have:

$$
s(S, \tau)=s\left(f_{2}, \tau\right) s\left(f_{3}, \tau\right)=(-1)(-1)=1
$$

Then the map $\varphi$ is trivial on $\Omega$ and the result follows.

Now we are able to prove theorem C. Let $S$ be a closed oriented surface in $\mathbf{R}^{4}$. Denote by $S_{i}$ the $i$-th component of $S$ and by $p_{i}$ the genus of $S_{i}$. Denote also by $u_{i}$ the element $\left(\delta / \pi_{i}\right)^{p_{i}-1}$. A $S$-state is characterized by the signs $e_{i}=\tau\left(S_{i}\right)$. So we have:

$$
\Psi(S)=\sum_{\tau} \widehat{S}(\tau)=\sum_{e_{*}} \prod_{i}\left(\left(\frac{\delta}{\pi}\right)^{p_{i}-1}\right)^{\left(e_{i}\right)}=\sum_{e_{*}} \prod_{i} u_{i}^{\left(e_{i}\right)}=\prod_{i}\left(u_{i}+\bar{u}_{i}\right)
$$

and the desired result follows from the obvious relation:

$$
\forall u \in R, \quad u+\bar{u}=\varepsilon(\delta u)
$$

6.14 Remark: We can extend the functor $\Psi$ to the category $\mathscr{L}^{\prime \prime}$ of decorated cobordisms of links. Consider a closed oriented surface $S$ in $\mathbf{R}^{4}$ decorated by $u$. This decoration sends each component $S_{i}$ of $S$ to an element $u_{i} \in R$. In this case we have:

$$
\Psi(S, u)=\prod_{i} \varepsilon\left(u_{i} \delta^{p_{i}} \pi^{1-p_{i}}\right)
$$

where $p_{i}$ is the genus of $S_{i}$. In any case $\Psi(S, u)$ doesn't depend on the embedding $S \subset \mathbf{R}^{4}$.

\section{References:}

[Bl] Christian Blanchet - An oriented model for Khovanov homology, Journal of Knot Theory and its Ramifications Vol 19, N² (2010), 291-312, math.GT/1405.7246.

[BM] Dror Bar Natan, Scott Morrison - The Karoubi Envelope and Lee's Degeneration of Khovanov Homology, Alg. Geom. Topol. 6 (2006), 1459-1469, math.GT/0606542. 
[BN1] Dror Bar Natan - On Khovanov's categorification of the Jones polynomial, Alg. Geom. Topol. 2 (2002), 337-370, math.QA/0201043.

[BN2] Dror Bar Natan - Khovanov's homology for tangles and cobordisms, Geom. Topol. 9 (2005), 1443-1499, math.GT/0410495.

[CC] Carmen Livia Caprau - sl(2) tangle homology with a parameter and singular cobordisms, Alg. Geom. Topol. 8 (2008), 729-756.

[CKSS] J. Scott Carter, Seiichi Kamada, Masahico Saito, Shin Satoh - A theorem of Sanderson on link bordisms in dimension 4, Alg. Geom. Topol. 1 (2001), 299-310.

[CMW] Davis Clark, Scott Morrison, Kevin Walker - Fixing the functoriality of Khovanov homology, Geom. Topol. 13 (2009), 1499-1582, math.GT/0701339.

[CS] J. Scott Carter, Masahico Saito - Reidemeister moves for surface isotopies and their interpretation as moves to movies, Journal of Knot Theory and its Ramifications Vol 2, №3 (1993), 251-284.

[Ja] Magnus Jacobson - An invariant of link cobordisms from Khovanov homology, Alg. Geom. Topol. 4 (2004), 1211-1254.

[Kh1] Mikhail Khovanov - A categorification of the Jones polynomial, Duke Math. J. Vol 101, N 3 (2000), 359-426, math.QA/9908171.

[Kh2] Mikhail Khovanov - A functor-valued invariant of tangles, Alg. Geom. Topol. 2 (2002), 665-741, math.QA/0103190.

[Kh3] Mikhail Khovanov - An invariant of tangle cobordism, Trans. Amer. Math. Soc. 358 (2006), 315-327.

[Kh4] Mikhail Khovanov - Link homology and Frobenius extensions, Fund. Math. 190 (2006), 179-190, math.QA/0411447.

[Ko] Joachim Kock - Frobenius algebras and 2D Topological Quantum Field Theories, LMS Student Texts 59 (2003), Cambridge University Press.

[Le] Eun Soo Lee - Khovanov's invariants for alternating links, math.GT/0210213 (2002).

[Ra1] Jacob Rasmussen - Khovanov homology and the slice genus, Invent. Math. 182 (2010), 419-447, math.GT/0402131.

[Ra2] Jacob Rasmussen - Khovanov's invariants for closed surfaces, math.GT/0502527 (2005).

[Ta] Kokoro Tanaka - Khovanov-Jacobsson numbers and invariants of surfaceknots derived from Bar-Natan's theory, Proc. Amer. Math. Soc. 134 no. 12 (2006), 3685-3689. 